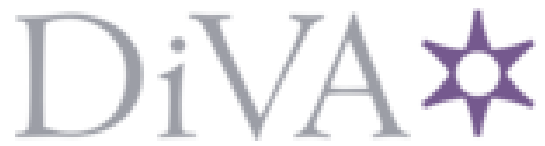

http://www.diva-portal.org

This is the published version of a paper published in ACM Transactions on Computational Logic.

Citation for the original published paper (version of record):

Goranko, V., Kuusisto, A., Rönnholm, R. (2018)

Game-Theoretic Semantics for Alternating-Time Temporal Logic

ACM Transactions on Computational Logic, 19(3): 17

https://doi.org/10.1145/3179998

Access to the published version may require subscription.

N.B. When citing this work, cite the original published paper.

Permanent link to this version:

http://urn.kb.se/resolve?urn=urn:nbn:se:su:diva-161056 


\section{Game-Theoretic Semantics for Alternating-Time Temporal Logic}

VALENTIN GORANKO, Stockholm University, and University of Johannesburg (visiting professorship)

ANTTI KUUSISTO, University of Bremen

RAINE RÖNNHOLM, University of Tampere

We introduce several versions of game-theoretic semantics (GTS) for Alternating-Time Temporal Logic (ATL). In GTS, truth is defined in terms of existence of a winning strategy in a semantic evaluation game. Thus, the game-theoretic perspective appears in the framework of ATL on two semantic levels: on the object level in the standard semantics of the strategic operators and on the meta-level, where game-theoretic logical semantics is applied to ATL. We unify these two perspectives into semantic evaluation games specially designed for ATL. The game-theoretic perspective enables us to identify new variants of the semantics of ATL based on limiting the time resources available to the verifier and falsifier in the semantic evaluation game. We introduce and analyze an unbounded and (ordinal) bounded GTS and prove these to be equivalent to the standard (Tarski-style) compositional semantics. We show that, in bounded GTS, truth of ATL formulae can always be determined in finite time, that is, without constructing infinite paths. We also introduce a nonequivalent finitely bounded semantics and argue that it is natural from both logical and game-theoretic perspectives.

CCS Concepts: • Theory of computation $\rightarrow$ Logic and verification; Modal and temporal logics; • Computing methodologies $\rightarrow$ Multiagent systems;

Additional Key Words and Phrases: Logic and game theory, logics for agents and multiagent systems, argumentation-based dialogue and protocols

ACM Reference format:

Valentin Goranko, Antti Kuusisto, and Raine Rönnholm. 2018. Game-Theoretic Semantics for AlternatingTime Temporal Logic. ACM Trans. Comput. Logic 19, 3, Article 17 (August 2018), 38 pages.

https://doi.org/10.1145/3179998

\section{INTRODUCTION}

Alternating-Time Temporal Logic ATL was introduced in Alur et al. (2002) as a multiagent extension of the branching-time temporal logic CTL. The semantics of ATL is defined over multiagent transition systems, also known as concurrent game models, in which agents take simultaneous actions at the current state and the resulting collective action determines the subsequent transition to a

This article is an extended and revised version of the conference paper (Goranko et al. 2016).

The work of Valentin Goranko was supported by a research grant no. 2015-04388 of the Swedish Research Council. The work of Antti Kuusisto was supported by the ERC grant no. 647289 "CODA."

Authors' addresses: V. Goranko, Department of Philosophy, Stockholm University, Universitetsvägen 10 D, Frescati, SE - 10691, Stockholm Sweden; email: valentin.goranko@philosophy.su.se; A. Kuusisto, University of Bremen, FB03, Bibliothekstr. 1, 28359, Bremen, Germany; email: kuusisto@uni-bremen.de; R. Rönnholm, Faculty of Natural Sciences, 33014 University of Tampere, Finland; email: raine.ronnholm@uta.fi.

Permission to make digital or hard copies of all or part of this work for personal or classroom use is granted without fee provided that copies are not made or distributed for profit or commercial advantage and that copies bear this notice and the full citation on the first page. Copyrights for components of this work owned by others than ACM must be honored. Abstracting with credit is permitted. To copy otherwise, or republish, to post on servers or to redistribute to lists, requires prior specific permission and/or a fee. Request permissions from permissions@acm.org.

(c) 2018 ACM 1529-3785/2018/08-ART17 $\$ 15.00$

https://doi.org/10.1145/3179998

ACM Transactions on Computational Logic, Vol. 19, No. 3, Article 17. Publication date: August 2018. 
successor state. The logic ATL and its extension ATL* have gradually become the most popular logical formalisms for reasoning about strategic abilities of agents in synchronous multiagent systems.

Game-theoretic semantics (GTS) of logical languages has a rich history going back to Hintikka (1973), Lorenzen (1961), and others. For an overview of the topic, see Hintikka and Sandu (1997). In GTS, truth of a logical formula $\varphi$ is determined in a formal debate between two players, Eloise and Abelard. Eloise is trying to verify $\varphi$, while Abelard is opposing her and trying to falsify it. Each logical connective in the language is associated with a related rule in the game. The framework of GTS has turned out to be particularly useful for the purpose of defining variants of semantic approaches to different logics. For example, the IF-logic of Hintikka and Sandu (1989) is an extension of first-order logic, which was originally developed using GTS. Also, the game-theoretic approach to semantics has led to new methods for solving decision problems of logics, for example, via using parity games for the $\mu$-calculus. The close connection between $\mu$-calculus model checking and parity games was first discussed in Emerson and Jutla (1988).

Here, we introduce game-theoretic semantics for ATL. In our framework, the rules corresponding to strategic operators involve scenarios in which Eloise and Abelard are both controlling (leading) coalitions of agents with opposing objectives. The perspective offered by GTS enables us to develop novel approaches to ATL based on different time resources available to the players. In unbounded GTS, a coalition trying to verify an until formula is allowed to continue without a time limit, the price of an infinite play being a loss in the game. In bounded GTS, the coalition must commit to finishing in finite time by submitting an ordinal number in the beginning of the game. That ordinal controls the available time resources in the game and guarantees a finite play. Notably, even safety games (for always formulae) are evaluated in finite time; thus, the bounded and unbounded approaches to GTS are conceptually very different. However, despite the differences between the two semantics, we show that they are, in fact, equivalent to the standard compositional (i.e., Tarski-style) semantics of ATL and, therefore, also to each other.

We also introduce a restricted variant of the bounded GTS, called finitely bounded GTS, in which the ordinals controlling the time flow must always be finite. This is a particularly simple system of semantics in which the players will always announce the ultimate (always finite) duration of the game before the game begins. We show that the finitely bounded GTS is equivalent to the standard ATL semantics on image-finite models and, therefore, provides an alternative approach to ATL sufficient for most practical purposes. It is worth noting here that the difference between the finitely bounded and unbounded semantics is conceptually directly linked to the difference between for loops and while loops. Since the finitely bounded semantics is new, we also develop an equivalent (over all models) Tarski-style semantics for it.

For all systems of game-theoretic semantics studied here, we show that positional strategies suffice in the perfect information setting for ATL. In the framework of unbounded semantics, this means that strategies depend on the current state only. In the case of bounded and finitely bounded semantics, strategies may additionally depend on the value of the ordinal guiding the time flow of the game. As a by-product of our semantic investigations, we also introduce and study a range of new game-theoretic notions that are of interest also outside ATL, such as, for example, timed strategies, $n$-canonical strategies, and $\infty$-canonical strategies.

There are several related works concerning both ATL and its extensions as well as gametheoretic semantics. We mention here some such papers. ${ }^{1}$

- Imposing explicit time bounds on temporal operators in logical specification languages has often been done syntactically in the context of quantitative temporal reasoning; see, for

\footnotetext{
${ }^{1}$ We thank an anonymous reviewer for pointing out many of these references.
} 
example, Emerson et al. (1992). Typically, that amounts to, for example, replacing $\mathrm{F} q$ by a disjunction $q \vee \mathrm{F} q \vee \ldots \vee \mathrm{F}^{k} q$, abbreviated by $\mathrm{F}^{k} q$, for $k \in \mathbb{N}$, that imposes an explicit and uniform bound of $k$ steps for satisfying the eventuality $q$.

- Imposing time bounds in the semantics of temporal operators has also been proposed in several contexts, most notably in relation to finitary fairness (Alur and Henzinger 1998) and promptness in LTL (Kupferman et al. 2007) as well as in omega-regular and parity games (Chatterjee et al. (2009), Almagor et al. (2010), Mogavero et al. (2013), and Aminof et al. (2016)). The idea of promptness (in LTL) is to replace the unbounded eventuality operator $F$ by a "bounded-eventually" operator $F_{p}$ for which an upper bound for the satisfaction of the eventuality is explicitly specified in the semantics.

-GTS-like approaches have been used to solve decision problems of, for example, fragments of Strategy Logic (especially with respect to the so-called "behavioral semantics") in Mogavero et al. (2014) and Cermák et al. (2015).

- Hella et al. (2017) discuss an alternative game-theoretic semantics for the modal $\mu$-calculus based on imposing time bounds on the fixed-point operators.

- In recent work (Goranko et al. 2017a), we introduce and study the variant of CTL with finitely bounded semantics. We show that it is a proper sublogic (in terms of validities) of standard CTL. We also show that it lacks the finite model property, but its satisfiability problem is nevertheless decidable (shown via finitary tableaux methods) and still EXPTIMEcomplete. In Goranko et al. (2018), we extend these results for ATL and also provide an infinitary complete axiomatization for ATL with finitely bounded semantics.

The main contributions of this article are twofold: the development of game-theoretic semantics for ATL and the introduction of new resource-sensitive versions of logics for multiagent strategic reasoning. The latter relates conceptually to the study of other resource-bounded versions of ATL; see, for example, Alechina et al. (2011), Monica et al. (2011), and Alechina et al. (2015). We note that some of our technical results could be obtained using alternative methods from coalgebraic modal logic. We discuss this issue in more detail in the concluding Section 5.3.

The structure of the article is as follows. After the preliminaries in Section 2, we develop the bounded and unbounded GTS in Section 3. We analyze these frameworks in Section 4, where we show, inter alia, that the two game-theoretic systems, bounded and unbounded, are equivalent. In Section 5, we compare the game-theoretic and standard Tarski-style semantics and establish the abovementioned equivalences between them. After brief concluding remarks, we provide an appendix with some technical proofs.

\section{PRELIMINARIES}

Here, we define concurrent game models as well as the syntax and standard compositional semantics of ATL. For detailed background on these, see Alur et al. (2002) or Goranko and van Drimmelen (2006).

Definition 2.1. A concurrent game model (CGM) $\mathcal{M}$ is a tuple

$$
\text { (Agt, St, } \Pi, \text { Act, } d, o, v)
$$

that consists of the following nonempty sets:

-agents $\mathbb{A g t}=\{1, \ldots, k\}$,

-states St,

-proposition symbols $\Pi$,

-actions Act,

and the following functions: 
- an action function $d: \mathbb{A g t} \times \mathrm{St} \rightarrow(\mathcal{P}($ Act $) \backslash\{\emptyset\})$, which assigns to each agent at each state a nonempty set of actions available to the agent at that state;

- a transition function $o$, which assigns to each state $q \in$ St and action profile $\vec{\alpha}$ at $q$ (where $\vec{\alpha}$ is a tuple of actions $\vec{\alpha}=\left(\alpha_{1}, \ldots, \alpha_{k}\right)$ such that $\alpha_{i} \in d(i, q)$ for each $\left.i \in \mathbb{A g t}\right)$, a unique outcome state $o(q, \vec{\alpha})$;

- and a valuation function $v: \Pi \rightarrow \mathcal{P}(\mathrm{St})$.

Sets of agents $A \subseteq \mathbb{A g t}$ are also called coalitions. The complement $\bar{A}=\mathbb{A g t} \backslash A$ of a coalition $A$ is called the opposing coalition (of $A$ ). We also define the set of action tuples that are available to a coalition $A$ at a state $q \in \mathrm{St}$ :

$$
\operatorname{action}(A, q):=\left\{\left(\alpha_{i}\right)_{i \in A} \mid \alpha_{i} \in d(i, q) \text { for each } i \in A\right\} .
$$

Definition 2.2. Let $\mathcal{M}=(\mathbb{A g t}, \mathrm{St}, \Pi, \mathrm{Act}, d, o, v)$ be a concurrent game model. A (positional) strategy $y^{2}$ for an agent $a \in \mathbb{A g t}$ is a function $s_{a}:$ St $\rightarrow$ Act such that $s_{a}(q) \in d(a, q)$ for each $q \in$ St. A collective strategy $S_{A}$ for $A \subseteq \mathbb{A g t}$ is a tuple of individual strategies, one for each agent in $A$. A path in $\mathcal{M}$ is a sequence of states $\Lambda$ such that $\Lambda[n+1]=o(\Lambda[n], \vec{\alpha})$ for some action profile $\vec{\alpha}$ for $\Lambda[n]$, where $\Lambda[n]$ is the $n$th state in $\Lambda(n \in \mathbb{N})$. The function paths $\left(q, S_{A}\right)$ returns the set of all paths generated when the agents in $A$ play according to $S_{A}$, beginning from the state $q$.

Alternating-time temporal logic ATL, introduced in Alur et al. (2002), is a logic suitable for specifying and verifying qualitative objectives of players and coalitions in concurrent game models. The main syntactic construct of ATL is a formula of type $\langle\langle A\rangle \varphi$, intuitively meaning that the coalition $A$ has a collective strategy to guarantee the satisfaction of the objective $\varphi$ on every play enabled by that strategy. The syntax of ATL is defined as follows ${ }^{3}$

$$
\varphi::=p|\neg \varphi|(\varphi \vee \varphi)|\langle A \lambda\rangle \mathrm{X} \varphi|\langle A \lambda\rangle \varphi \mathrm{U} \varphi \mid\langle A \lambda\rangle \varphi \mathrm{R} \varphi,
$$

where $A \subseteq \mathbb{A}$ gt and $p \in \Pi$. Other Boolean connectives are defined as usual; the combined operators $\langle\langle A\rangle \mathrm{F} \varphi$ and $\langle\langle A\rangle \mathrm{G} \varphi$ are defined, respectively, by $\langle\langle A\rangle\rceil \mathrm{U} \varphi$ and $\langle\langle A\rangle \perp \mathrm{R} \varphi$.

Definition 2.3. Let $\mathcal{M}=(\mathbb{A g t}, \mathrm{St}, \Pi$, Act, $d, o, v)$ be a CGM, $q \in \mathrm{St}$ a state, and $\varphi$ an ATL formula. Truth of $\varphi$ in $\mathcal{M}$ at $q$, denoted by $\mathcal{M}, q \mid \varphi$, is defined as follows:

$-\mathcal{M}, q \mid p$ iff $q \in v(p)($ for $p \in \Pi)$.

$-\mathcal{M}, q \mid \neg \psi$ iff $\mathcal{M}, q \notin \psi$.

$-\mathcal{M}, q \mid=\psi \vee \theta$ iff $\mathcal{M}, q \mid \psi \psi$ or $\mathcal{M}, q \mid=\theta$.

$-\mathcal{M}, q \mid=\left\langle\langle A\rangle \mathrm{X} \psi\right.$ iff there exists a collective strategy $S_{A}$ such that for each $\Lambda \in \operatorname{paths}\left(q, S_{A}\right)$, we have that $\mathcal{M}, \Lambda[1] \mid=\psi$.

$-\mathcal{M}, q \mid=\left\langle\langle A\rangle \psi \cup \theta\right.$ iff there exists a collective strategy $S_{A}$ such that for each $\Lambda \in$ paths $\left(q, S_{A}\right)$, there is $i \geq 0$ such that $\mathcal{M}, \Lambda[i] \mid=\theta$ and $\mathcal{M}, \Lambda[j] \mid \psi$ for every $j<i$.

$-\mathcal{M}, q \mid=\left\langle\langle A\rangle \psi \mathrm{R} \theta\right.$ iff there exists a collective strategy $S_{A}$ such that for each $\Lambda \in \operatorname{paths}\left(q, S_{A}\right)$ and $i \geq 0$, we have that $\mathcal{M}, \Lambda[i] \mid=\theta$ or there is $j<i$ such that $\mathcal{M}, \Lambda[j] \mid=\psi$.

\section{GAME-THEORETIC SEMANTICS}

In this section, we introduce three versions of evaluation games for ATL: unbounded, (ordinal) bounded, and finitely bounded evaluation games. These games will ultimately enable us to define three different versions of game-theoretic semantics for ATL.

\footnotetext{
${ }^{2}$ Unless otherwise specified, a strategy hereafter will mean a positional and deterministic strategy.

${ }^{3}$ The operator R (Release) was not part of the original syntax of ATL but has been commonly added later.
} 


\subsection{Unbounded Evaluation Games}

Given a CGM $\mathcal{M}$, a state $q_{i n}$, and a formula $\varphi$, the evaluation game $\mathcal{G}\left(\mathcal{M}, q_{i n}, \varphi\right)$ is intuitively a debate between two opponents, Eloise (E) and Abelard (A), about whether the formula $\varphi$ is true at the state $q_{i n}$ in the model $\mathcal{M}$. Eloise claims that $\varphi$ is true; thus, she adopts (initially) the role of a verifier in the game. Abelard tries to prove the formula false; thus, he is (initially) the falsifier. These roles can swap in the course of the game when negations are encountered in the formula to be evaluated. Truth of an ATL formula $\varphi$ in $\mathcal{M}$ at $q_{\text {in }}$ will be defined as existence of a winning strategy for $\mathrm{E}$ in the game $\mathcal{G}\left(\mathcal{M}, q_{i n}, \varphi\right)$.

We will often use the following notation: if $\mathbf{P} \in\{\mathrm{A}, \mathrm{E}\}$, then $\overline{\mathbf{P}}$ denotes the opponent of $\mathbf{P}$, that is, $\overline{\mathbf{P}} \in\{\mathrm{A}, \mathrm{E}\} \backslash\{\mathbf{P}\}$.

Definition 3.1. Let $\mathcal{M}=(\mathbb{A g t}, \mathrm{St}, \Pi$, Act, $d, o, v)$ be a CGM, $q_{i n} \in \mathrm{St}$ and $\varphi$ an ATL formula. The unbounded evaluation game $\mathcal{G}\left(\mathcal{M}, q_{i n}, \varphi\right)$ between the players $\mathbf{A}$ and $\mathbf{E}$ is defined as follows.

-A position of the game is a tuple $\operatorname{Pos}=(\mathbf{P}, q, \psi)$ where $\mathbf{P} \in\{\mathbf{A}, \mathbf{E}\}, q \in \mathrm{St}$, and $\psi$ is a subformula of $\varphi$. The initial position of the game is $\operatorname{Pos}_{0}:=\left(\mathbf{E}, q_{i n}, \varphi\right)$.

-In every position $(\mathbf{P}, q, \psi)$, the player $\mathbf{P}$ is called the verifier and $\overline{\mathbf{P}}$ the falsifier for that position.

- Each position of the game is associated with a rule. The rules for positions in which the related formula is either a proposition symbol or has a Boolean connective as its main connective are defined as follows.

(1) If $\operatorname{Pos}_{i}=(\mathbf{P}, q, p)$, where $p \in \Pi$, then $\operatorname{Pos}_{i}$ is called an ending position of the evaluation game. If $q \in v(p)$, then $\mathbf{P}$ wins the evaluation game. Otherwise, $\overline{\mathbf{P}}$ wins.

(2) Let $\operatorname{Pos}_{i}=(\mathbf{P}, q, \neg \psi)$. The game then moves to the next position $\operatorname{Pos}_{i+1}=(\overline{\mathbf{P}}, q, \psi)$.

(3) Let $\operatorname{Pos}_{i}=(\mathbf{P}, q, \psi \vee \theta)$. Then, the player $\mathbf{P}$ decides whether the next position $\operatorname{Pos}_{i+1}$ is $(\mathbf{P}, q, \psi)$ or $\operatorname{Pos}_{i+1}=(\mathbf{P}, q, \theta)$.

In order to deal with the strategic operators, we now define a one-step game, denoted by step $(\mathbf{P}, A, q)$, where $A \subseteq \mathbb{A g t}$. This game consists of the following two actions.

(i) First, $\mathrm{P}$ chooses an action $\alpha_{i} \in d(i, q)$ for each $i \in A$.

(ii) Then, $\overline{\mathbf{P}}$ chooses an action $\alpha_{i} \in d(i, q)$ for each $i \in \bar{A}$.

The resulting state of the one-step game $\operatorname{step}(\mathrm{P}, A, q)$ is the state

$$
q^{\prime}:=o\left(q, \alpha_{1}, \ldots, \alpha_{k}\right)
$$

arising from the combined action of the agents. We now use the one-step game to define how the evaluation game proceeds from positions in which the formula is of type $\langle\langle A\rangle\rangle \mathrm{X} \psi$

(4) Let $\operatorname{Pos}_{i}=(\mathbf{P}, q,\langle\langle A\rangle\rangle \chi)$. The next position $\operatorname{Pos}_{i+1}$ is $\left(\mathbf{P}, q^{\prime}, \psi\right)$, where $q^{\prime}$ is the resulting state of $\operatorname{step}(\mathbf{P}, A, q)$.

The rules for the other strategic operators are obtained by iterating the one-step game. For this purpose, we now define the embedded game

$$
\mathrm{G}:=\mathbf{g}\left(\mathbf{V}, \mathbf{C}, A, q_{0}, \psi_{\mathrm{C}}, \psi_{\overline{\mathrm{C}}}\right),
$$

where both $\mathbf{V}, \mathbf{C} \in\{\mathbf{E}, \mathbf{A}\}, A$ is a coalition, $q_{0}$ a state, and $\psi_{\mathbf{C}}$ and $\psi_{\overline{\mathbf{C}}}$ are formulae. The player $\mathbf{V}$ is called the verifier (of the embedded game) and $\mathrm{C}$ the controller. These players may be, but need not be, the same. We let $\overline{\mathbf{V}}$ and $\overline{\mathbf{C}}$ denote the opponents of $\mathbf{V}$ and $\mathbf{C}$, respectively.

The embedded game $\mathrm{G}$ starts from the initial state $q_{0}$ and proceeds from any state $q$ according to the following rules, applied in the order given below, until an exit position is reached. 
(i) $\mathbf{C}$ may end the game at the exit position $\left(\mathrm{V}, q, \psi_{\mathrm{C}}\right)$.

(ii) $\overline{\mathrm{C}}$ may end the game at the exit position $\left(\mathrm{V}, q, \psi_{\overline{\mathrm{C}}}\right)$.

(iii) If the game has not ended by the above rules, the one-step game $\operatorname{step}(\mathrm{V}, A, q)$ is played to produce a resulting state $q^{\prime}$. The embedded game is continued from the state $q^{\prime}$.

If the embedded game $\mathrm{G}$ continues an infinite number of rounds, the controller $\mathrm{C}$ loses the entire evaluation game $\mathcal{G}\left(\mathcal{M}, q_{i n}, \varphi\right)$. Otherwise, the evaluation game resumes from the exit position of the embedded game.

We now define the rules of the evaluation game for the remaining strategic operators as follows:

(5) Let $\operatorname{Pos}_{i}=(\mathbf{P}, q,\langle\langle A\rangle\rangle \cup \theta)$. The next position $\operatorname{Pos}_{i+1}$ is the exit position of the embedded game $\mathbf{g}(\mathbf{P}, \mathbf{P}, A, q, \theta, \psi)$. (Note the order of the formulae $\theta$ and $\psi$.)

(6) Let $\operatorname{Pos}_{i}=\left(\mathbf{P}, q,\langle\langle A\rangle \psi \psi \mathrm{R} \theta)\right.$. The next position $\operatorname{Pos}_{i+1}$ is the exit position of the embedded game $\mathbf{g}(\mathbf{P}, \overline{\mathbf{P}}, A, q, \theta, \psi)$.

This completes the definition of the evaluation game.

Remark 3.2. The evaluation games we have defined make use of special subgames-the embedded games. The reason for distinguishing these subgames from the evaluation games is purely technical and could be avoided. The distinction will help us organize and simplify proofs later in the article. The most natural way of thinking of the evaluation games and the embedded subgames is that they simply form a single game used for evaluating formulae of ATL and the separation of the embedded games matters only in our reasoning about the evaluation games, not in using these games for evaluating formulae.

We can say that the embedded game for a formula $\langle\langle A\rangle \psi \cup \theta$ is an eventuality game and the embedded game for $\langle\langle A\rangle\rangle \mathrm{R} \theta$ a safety game. Intuitively, the embedded game $\mathbf{g}\left(\mathbf{V}, \mathbf{C}, A, q, \psi_{\mathbf{C}}, \psi_{\overline{\mathbf{C}}}\right)$ can be seen as a "simultaneous reachability game" in which both players have a goal they are trying to reach before the opponent reaches one's goal. The verifier $\mathrm{V}$ leads the coalition $A$ and the falsifier $\overline{\mathbf{V}}$ leads the opposing coalition $\bar{A}$. The goal of each of $\mathbf{V}$ and $\overline{\mathbf{V}}$ is defined by a formula. When $\mathbf{V}=\mathbf{C}$, the goal of $\mathbf{V}$ is to verify $\psi_{\mathbf{C}}$ and the goal of $\overline{\mathbf{V}}$ is to falsify $\psi_{\overline{\mathrm{C}}}$, where verifying $\psi_{\mathrm{C}}$ corresponds to reaching a state in which $\psi_{\mathrm{C}}$ holds and falsifying $\psi_{\overline{\mathrm{C}}}$ corresponds to reaching the complement of the set of states in which $\psi_{\overline{\mathbf{C}}}$ holds. When $\mathbf{V}=\overline{\mathbf{C}}$, the goal of $\mathbf{V}$ is to verify $\psi_{\overline{\mathbf{C}}}$ and that of $\overline{\mathbf{V}}$ is to falsify $\psi_{\mathrm{C}}$. Both players $\mathbf{V}$ and $\overline{\mathbf{V}}$ have the possibility to end the game when they believe that they have reached their goal. However, the controller is responsible for ending the embedded game in finite time and will lose if the game continues infinitely long. If both players reach their targets at the same time, the controller $\mathrm{C}$ has the priority to end the embedded game first.

Ending the embedded game can be seen as "making a claim" that would verify or falsify the temporal formula being evaluated in the embedded game. For example, in an embedded game for $\langle A\rangle\rangle \psi \cup \theta$, the verifier $\mathbf{V}$ may claim at any state $q$ that $\theta$ is true at $q$ and, thus, $\psi \cup \theta$ has been verified. The opponent $\overline{\mathrm{V}}$ can challenge this claim; thus, the claim is evaluated by continuing the evaluation game from the exit position $(\mathbf{V}, q, \theta)$. Similarly, $\overline{\mathbf{V}}$ may claim that $\psi$ is not true at $q$ and, thus, $\psi \cup \theta$ has been falsified. Then, $\mathrm{V}$ must defend the truth of $\psi$ from the exit position $(\mathbf{V}, q, \psi)$.

It is worth noting that, even though the coalitions in ATL operate concurrently, the verifier V in the embedded is forced to make the choices for the coalition in every round before the falsifier $\overline{\mathbf{V}}$; thus, $\overline{\mathrm{V}}$ has the advantage of making choices after $\mathrm{V}$ has made $\mathrm{V}$ 's choices. Therefore, the evaluation games are fully turn based (which can be beneficial in some technical contexts). Let us consider how this is reflected in the standard compositional semantics of ATL (Definition 2.3). If the formula $\langle A\rangle\rangle \Phi$ is true, then, by compositional semantics, $A$ has a collective strategy $S_{A}$ to enforce 
$\Phi$ regardless of the actions chosen by $\bar{A}$. Hence, the strategy $S_{A}$ will work even if the opposing coalition $\bar{A}$ could choose actions after seeing the actions chosen by $A$. If $\langle\langle A\rangle \Phi$ is not true, then, by compositional semantics, such a strategy $S_{A}$ simply does not exist. However, in the embedded game, it is the "responsibility" of the falsifier $\overline{\mathbf{V}}$ to create a path, where $\Phi$ does not hold, by leading the opposing coalition $\bar{A}$ and being able to choose actions for it after seeing those chosen for $A$.

If we compare the intuitive properties of our evaluation game with those of the standard compositional semantics, we notice that the strategic abilities of coalitions $A \subseteq \mathbb{A g t}$ are analyzed from a different perspective. The standard compositional semantics treats strategies explicitly by quantifying over collective strategies $S_{A}$. In contrast, the verifier and falsifier simply play the evaluation game step by step by controlling the coalitions $A$ and $\bar{A}$, respectively, and the verifier does not have to announce any strategy for $A$. Still, in GTS, the strategies also need to be treated explicitly when we consider which of the players has a winning strategy in the evaluation game.

Last, in the compositional semantics, temporal formulae are always evaluated on infinite paths, while in the evaluation game a path is constructed only up to the extent needed for verifying (or falsifying) a formula. In the unbounded evaluation game, infinite paths may still be constructed, but only if the controller continues the embedded game for infinitely many rounds (and thus loses the game).

In the next section, we define a bounded version of the evaluation game in which only finite paths need to be constructed.

\subsection{Bounded Evaluation Games}

The difference between bounded and unbounded evaluation games is that, in the bounded case, embedded games are associated with a time limit. In the beginning of a bounded evaluation game, the controller must announce some possibly infinite ordinal $\gamma$ that will decrease in each round. This will guarantee that the embedded game (and, in fact, the entire evaluation game) will end after a finite number of rounds.

Bounded evaluation games $\mathcal{G}\left(\mathcal{M}, q_{i n}, \varphi, \Gamma\right)$ have an additional parameter $\Gamma$, which is an ordinal that fixes a strict upper bound for the ordinals that the players can announce during the related embedded games. As we will see further, different values of $\Gamma$ give rise to different evaluation games and, thus, lead to different game-theoretic semantics.

Definition 3.3. Let $\mathcal{M}$ be a CGM, $q_{i n} \in \mathrm{St}, \varphi$ an ATL formula, and $\Gamma$ an ordinal. The bounded evaluation game (or $\Gamma$-bounded evaluation game) $\mathcal{G}\left(\mathcal{M}, q_{i n}, \varphi, \Gamma\right)$ is defined in the same way as the unbounded evaluation game $\mathcal{G}\left(\mathcal{M}, q_{i n}, \varphi\right)$, the only difference between the two games being the treatment of until formulae and release formulae. In the bounded case, these formulae are treated as follows.

Let $\mathbf{G}=\mathbf{g}\left(\mathbf{V}, \mathbf{C}, A, q_{0}, \psi_{\mathbf{C}}, \psi_{\overline{\mathrm{C}}}\right)$ be an embedded game that arises from a position Pos in $\mathcal{G}\left(\mathcal{M}, q_{i n}, \varphi\right)$. In the same position, Pos in the bounded evaluation game $\mathcal{G}\left(\mathcal{M}, q_{i n}, \varphi, \Gamma\right)$, the player $\mathrm{C}$ first chooses some ordinal $\gamma_{0}<\Gamma$ to be the initial time limit for the embedded game $\mathrm{G}$. This choice gives rise to a bounded embedded game that is denoted by $\mathrm{G}\left[\gamma_{0}\right]$ and played in the way described below.

A configuration of $\mathrm{G}\left[\gamma_{0}\right]$ is a pair $(\gamma, q)$, where $\gamma$ is a (possibly infinite) ordinal called the current time limit and $q \in$ St a state called the current state. The bounded embedded game $\mathrm{G}\left[\gamma_{0}\right]$ starts from the initial configuration $\left(\gamma_{0}, q_{0}\right)$ and proceeds from any configuration $(\gamma, q)$ according to the following rules, applied in the given order.

(i) If $\gamma=0$, the game ends at the exit position $\left(\mathrm{V}, q, \psi_{\mathrm{C}}\right)$.

(ii) $\mathrm{C}$ may end the game at the exit position $\left(\mathrm{V}, q, \psi_{\mathrm{C}}\right)$. 
(iii) $\overline{\mathrm{C}}$ may end the game at the exit position $\left(\mathrm{V}, q, \psi_{\overline{\mathrm{C}}}\right)$.

(iv) If the game has not ended due to the previous rules, then $\operatorname{step}(\mathrm{V}, A, q)$ is played in order to produce a resulting state $q^{\prime}$. Then, the bounded embedded game continues from the configuration $\left(\gamma^{\prime}, q^{\prime}\right)$, where $\gamma^{\prime}=\gamma-1$ if $\gamma$ is a successor ordinal and, if $\gamma$ is a limit ordinal, then $\gamma^{\prime}$ is an ordinal smaller than $\gamma$ and chosen by $\mathrm{C}$.

We denote the set of configurations in $\mathrm{G}\left[\gamma_{0}\right]$ by $\operatorname{Conf}_{\mathrm{G}\left[\gamma_{0}\right]}$. After the bounded embedded game $\mathrm{G}\left[\gamma_{0}\right]$ has reached an exit position-which it will, since ordinals are well founded-the evaluation game resumes from the exit position of the embedded game.

It is clear that bounded evaluation games end after a finite number of rounds because bounded embedded games do. Note that if time limits are infinite ordinals, they do not directly determine the number of rounds left in the game, but instead they are related to the game duration in a more abstract way.

It is also worth noting here that different ways of using ordinals in game-theoretic considerations go way back. An example of an important and relatively early reference is Smith (1966), which contains references to even earlier related articles.

It is instructive to analyze embedded games independently of evaluation games. An embedded game of the form $\mathrm{G}=\mathbf{g}\left(\mathbf{V}, \mathbf{C}, A, q_{0}, \psi_{\mathbf{C}}, \psi_{\overline{\mathrm{C}}}\right)$ can be played without a time limit, as in unbounded evaluation games, or it can be given some time limit $\gamma_{0}$ as a parameter, which leads to the related bounded embedded game $G\left[\gamma_{0}\right]$. When we use the plain notation $G$ (as opposed to $G\left[\gamma_{0}\right]$ ), we always assume that the embedded game $\mathrm{G}$ is not bounded. We sometimes emphasize this by calling $\mathrm{G}$ an unbounded embedded game. We will study the properties of embedded games in Section 4.

Let $\omega$ denote the smallest infinite ordinal. Evaluation games of the form

$$
\mathcal{G}\left(\mathcal{M}, q_{i n}, \varphi, \omega\right)
$$

constitute a particularly interesting subclass of bounded evaluation games. We call the games in this class finitely bounded evaluation games. In these games, only finite time limits can be announced for bounded embedded games.

Note that in Definition 3.3 we could handle successor ordinals in the same way as limit ordinals. That is, the controller should choose any ordinal $\gamma^{\prime}$ smaller than the current time limit $\gamma$ also when $\gamma$ is a successor ordinal. However, it easy to see that, in this case, the best choice for the controller is to always choose $\gamma^{\prime}=\gamma-1$; thus, this step in the game would be unnecessary. Furthermore, with finitely bounded evaluation games, it is natural that the time limit is always lowered by one after every transition in the embedded game.

Remark 3.4. In this article, the temporal operators $\langle\langle A\rangle \mathrm{F}$ and $\langle\langle A\rangle \mathrm{G}$ are regarded as syntactic abbreviations. Therefore, the rules associated with them can be extracted from those above. We now define alternative rules that could be directly given to $\langle\langle A\rangle\rangle \mathrm{F}$ and $\langle\langle A\rangle\rangle \mathrm{G}$ in the finitely bounded evaluation games, resulting in an equivalent semantics. (The fact that this equivalence holds will ultimately be straightforward to observe.)

- Let $\operatorname{Pos}_{i}=(\mathbf{P}, q,\langle\langle A\rangle\rangle \mathrm{F} \psi)$. First, the player $\mathbf{P}$ chooses some $n \in \mathbb{N}$ and then the players iterate $\operatorname{step}(\mathbf{P}, A, q)$ for at most $n$ times. The player $\mathbf{P}$ may decide to stop at the current state $q^{\prime}$ after any number $m \leq n$ of iterations; then, the evaluation game is continued from $\operatorname{Pos}_{i+1}=\left(\mathbf{P}, q^{\prime}, \psi\right)$.

-Let $\operatorname{Pos}_{i}=(\mathbf{P}, q,\langle\langle A\rangle \mathrm{G} \psi)$. First, the player $\overline{\mathbf{P}}$ chooses some $n \in \mathbb{N}$; then, the players iterate step $(\mathbf{P}, A, q)$ for at most $n$ times. The player $\overline{\mathbf{P}}$ may decide to stop at the current state $q^{\prime}$ after any number $m \leq n$ of iterations; the evaluation game is then continued from $\operatorname{Pos}_{i+1}=$ $\left(\mathbf{P}, q^{\prime}, \psi\right)$. 
Similar (but not identical) rules could also be given to $\langle\langle A\rangle\rangle \mathrm{F}$ and $\langle\langle A\rangle \mathrm{G}$ in the frameworks based on unbounded and ordinal-bounded games.

\subsection{Game-Theoretic Semantics}

A strategy for a player $\mathbf{P} \in\{\mathbf{A}, \mathbf{E}\}$ will be defined below to be a function on game positions; in positions in which the player $\mathbf{P}$ is not required to make a move, the strategy of $\mathbf{P}$ will output a special value "any," the meaning of which is "any possible value." We will also use any for some other functions when the output is not relevant, for example, when defining a winning strategy, we may assign any for losing positions.

Definition 3.5. Let $\mathrm{G}=\mathbf{g}\left(\mathbf{V}, \mathbf{C}, A, q_{0}, \psi_{\mathbf{C}}, \psi_{\overline{\mathrm{C}}}\right)$ be an embedded game and $\mathbf{P} \in\{\mathbf{A}, \mathbf{E}\}$. A strategy for the player $\mathrm{P}$ in $\mathrm{G}$ is a function $\sigma_{\mathrm{P}}$ whose domain is $\mathrm{St}$ and whose range is specified below.

First, for any $q \in \mathrm{St}$, it is possible to define $\sigma_{\mathrm{P}}(q) \in\left\{\psi_{\mathrm{C}}, \psi_{\overline{\mathrm{C}}}\right\}$; then, $\sigma_{\mathrm{P}}$ instructs $\mathbf{P}$ to end the game at the state $q$. Here, it is required that if $\mathbf{P}=\mathbf{C}$, then $\sigma_{\mathbf{P}}(q)=\psi_{\mathbf{C}}$ and if $\mathbf{P}=\overline{\mathbf{C}}$, then $\sigma_{\mathbf{P}}(q)=\psi_{\overline{\mathbf{C}}}$. (Intuitively, if $\sigma_{\mathbf{P}}(q)=\psi \in\left\{\psi_{\mathbf{C}}, \psi_{\overline{\mathrm{C}}}\right\}$, then the strategy $\sigma_{\mathbf{P}}$ instructs $\mathbf{P}$ to claim that $\psi$ is true at $q$.)

If $\sigma_{\mathrm{P}}(q) \notin\left\{\psi_{\mathrm{C}}, \psi_{\overline{\mathrm{C}}}\right\}$, then the following conditions hold.

-If $\mathbf{P}=\mathbf{V}$, then $\sigma_{\mathbf{P}}(q)$ is a tuple of actions in action $(A, q)$ (to be chosen for $A$ ).

-If $\mathbf{P}=\overline{\mathbf{V}}$, then $\sigma_{\mathbf{P}}(q)$ is defined to be a response function $f: \operatorname{action}(A, q) \rightarrow \operatorname{action}(\bar{A}, q)$ that assigns a tuple of actions for $\bar{A}$ as a response to any tuple of actions chosen for $A$ (by the opposing player).

Let $\gamma_{0}$ be an ordinal. A strategy $\sigma_{\mathbf{P}}$ for $\mathbf{P}$ in $\mathrm{G}\left[\gamma_{0}\right]$ is defined in the same way as a strategy in $\mathbf{G}$, but the domain of this strategy is the set of all possible configurations $\operatorname{Conf}_{\mathrm{G}\left[\gamma_{0}\right]}$.

Note that strategies in embedded games are positional, that is, they depend only on the current state in the unbounded case and on the current configuration in the bounded case. We will see later that if strategies were allowed to depend on more information, such as the sequence of states played, the resulting semantic systems would be equivalent to the current ones.

Any strategy $\sigma_{\mathrm{P}}$ for an unbounded embedded game $\mathrm{G}$ can also be used in any bounded embedded game $\mathrm{G}\left[\gamma_{0}\right]$ : we simply use the same action $\sigma_{\mathrm{P}}(q)$ for each configuration $(\gamma, q) \in \mathrm{Conf}_{\mathrm{G}\left[\gamma_{0}\right]}$. In general, this does not work the other way around since a strategy $\sigma_{\mathrm{P}}$ that is defined for configurations may give different values for $(\gamma, q)$ and $\left(\gamma^{\prime}, q\right)$. However, if a strategy $\sigma_{\mathrm{P}}$ for a bounded embedded game $\mathrm{G}\left[\gamma_{0}\right]$ is independent of time limits (thus depends on states only), it can also be used in the unbounded embedded game $\mathrm{G}$. This observation will be crucial later when we compare bounded embedded games with the corresponding unbounded embedded games.

We next define the notion of strategy for evaluation games, using strategies for embedded games as substrategies. We first present the definition for unbounded evaluation games.

Definition 3.6. Let $\mathbf{P} \in\{\mathbf{A}, \mathbf{E}\}$. A strategy for player $\mathbf{P}$ in an unbounded evaluation game $\mathcal{G}=\mathcal{G}\left(\mathcal{M}, q_{i n}, \varphi\right)$ is a function $\Sigma_{\mathrm{P}}$ defined on the set of positions POS of $\mathcal{G}$ (with the range specified below) satisfying the following conditions.

(1) If $\operatorname{Pos}=(\mathbf{P}, q, \psi \vee \theta)$, then $\Sigma_{\mathbf{P}}(\operatorname{Pos}) \in\{\psi, \theta\}$.

(2) If $\operatorname{Pos}=\left(\mathbf{P}, q,\langle\langle A\rangle \mathrm{X} \psi)\right.$, then $\Sigma_{\mathbf{P}}(\mathrm{Pos})$ is a tuple of actions in action $(A, q)$ for the one-step game $\operatorname{step}(\mathbf{P}, A, q)$.

(3) If $\operatorname{Pos}=(\overline{\mathbf{P}}, q,\langle\langle A\rangle\rangle \mathrm{X} \psi)$, then $\sum_{\mathbf{P}}(\operatorname{Pos})$ is a response function $f: \operatorname{action}(A, q) \rightarrow$ $\operatorname{action}(\bar{A}, q)$ for the one-step game $\operatorname{step}(\mathrm{P}, A, q)$.

(4) Let $\operatorname{Pos}=(\mathbf{P}, q,\langle\langle A\rangle\rangle \psi \mathrm{T} \theta)$ or $\operatorname{Pos}=(\overline{\mathbf{P}}, q,\langle\langle A\rangle\rangle \psi \mathrm{T} \theta)$, where $\mathrm{T} \in\{\mathrm{U}, \mathrm{R}\}$. Then, $\Sigma_{\mathbf{P}}(\mathrm{Pos})$ is a strategy $\sigma_{\mathbf{P}}$ for $\mathbf{P}$ in the respective embedded game $\mathbf{g}(\mathrm{V}, \mathbf{C}, A, q, \theta, \psi)$.

(5) In all other cases, $\Sigma_{\mathbf{P}}(\mathrm{Pos})=$ any (the player $\mathbf{P}$ is not required to make a move). 
We say that the player $\mathbf{P}$ plays according to the strategy $\Sigma_{\mathrm{P}}$ in the evaluation game $\mathcal{G}$ if $\mathbf{P}$ makes choices in $\mathcal{G}$ according to that strategy. We then say that $\Sigma_{\mathrm{P}}$ is a winning strategy for $\mathrm{P}$ in $\mathcal{G}$ if $\mathrm{P}$ wins all plays of $\mathcal{G}$ where $\mathrm{P}$ plays according to that strategy.

We now define strategies for bounded evaluation games. This definition differs from the previous one in positions that lead to an embedded game. Here, one of the players must announce a time limit for the embedded game. The player announcing the time limit must also decide how to lower the time limit when a limit ordinal is reached: this will be done by a substrategy called a timer. Strategies of the other player may depend on the announced time limits.

Definition 3.7 (c.f. Remark 3.9). A strategy for player $\mathrm{P}$ in a bounded evaluation game $\mathcal{G}=$ $\mathcal{G}\left(\mathcal{M}, q_{i n}, \varphi, \Gamma\right)$ is defined as in Definition 3.6, with the exception of positions with until formulae and release formulae, which are treated as follows.

(4) Let $\operatorname{Pos}=(\mathbf{P}, q,\langle\langle A\rangle\rangle \psi \mathrm{T} \theta)$ or $\operatorname{Pos}=(\overline{\mathbf{P}}, q,\langle\langle A\rangle\rangle \psi \mathrm{T} \theta)$, where $\mathrm{T} \in\{\mathrm{U}, \mathrm{R}\}$, and let $\mathrm{G}=$ $\mathrm{g}(\mathrm{V}, \mathrm{C}, A, q, \theta, \psi)$ denote the embedded game related to Pos.

If $\mathbf{P}=\mathbf{C}$, then $\Sigma_{\mathbf{P}}(\mathrm{Pos})=\left(\gamma_{0}, t, \sigma_{\mathbf{P}}\right)$ such that the following conditions hold.

$-\gamma_{0}<\Gamma$ is an ordinal. It is the choice for the initial time limit and leads to the bounded embedded game $\mathrm{G}\left[\gamma_{0}\right]$.

$-t$ is a function, called timer, on pairs $(\gamma, q)$, where $\gamma \leq \gamma_{0}$ is a limit ordinal and $q \in$ St. The value $t(\gamma, q)$ must be an ordinal less than $\gamma$. The timer $t$ gives an instruction on how to lower the time limit $\gamma$ after a transition to $q$ has been made.

$-\sigma_{\mathbf{P}}$ is a strategy for $\mathbf{P}$ in $\mathbf{G}\left[\gamma_{0}\right]$.

Finally, if $\mathbf{P} \neq \mathbf{C}$, then $\Sigma_{\mathbf{P}}(\mathrm{Pos})$ is a function that maps any ordinal $\gamma_{0}<\Gamma$ to some strategy $\sigma_{\mathbf{P}, \gamma_{0}}$ for $\mathbf{P}$ in $\mathrm{G}\left[\gamma_{0}\right]$.

In finitely bounded evaluation games, only finite time limits $\gamma_{0}<\omega$ may be announced by the controller C. Since no limit ordinal can be reached, the timer $t$ can be omitted from the strategy in this special case.

Remark 3.8. As discussed in Remark 3.2, instead of considering embedded games as separate subgames of the evaluation game, they can be merged into the evaluation game. Thus, when considering the full evaluation game (including the embedded games), it is not necessary to make a distinction between positions and configurations. In this approach, we could only consider the strategy $\Sigma_{\mathbf{P}}$ for the evaluation game instead of having substrategies $\sigma_{\mathbf{P}}$ for related embedded games. Moreover, in the bounded case, the timer $t$ is given as a separate component together with $\sigma_{\mathrm{P}}$, but it could perhaps be more natural to make it a part of $\sigma_{\mathrm{P}}$.

However, this separation of evaluation games and embedded games, as well as the corresponding strategies, allows us to analyze the properties of embedded games in a modular fashion. The separation will also be very useful when comparing GTS with the standard compositional semantics since the verifier's strategies $\sigma_{\mathrm{V}}$ (without the timer) will then be very closely related to the collective strategies $S_{A}$ (recall Definition 2.3).

Remark 3.9. Note that in Definition 3.7 we allow, for technical convenience, the strategies for an embedded game $\mathrm{g}\left(\mathrm{V}, \mathrm{C}, A, q_{0}, \psi_{\mathrm{C}}, \psi_{\overline{\mathrm{C}}}\right)\left[\gamma_{0}\right]$ to depend on all parameters of the game in addition to the configurations $(q, \gamma)$ that can occur in the embedded game. Naturally, the parameters $\mathbf{V}, \mathbf{C}$, $A, \psi_{\mathrm{C}}$, and $\psi_{\overline{\mathrm{C}}}$ are all crucial information to the players, but for "genuinely positional" strategies, the initial state $q_{0}$ and the initial time limit $\gamma_{0}$ should only be available to a player when the game is in the initial configuration $\left(q_{0}, \gamma_{0}\right)$. However, we can show that $q_{0}$ and $\gamma_{0}$ are indeed unnecessary 
bits of information for the players when the game is not in the initial configuration. This follows from Proposition 4.10, which we present later.

Different choices for time limit bounds $\Gamma$ give rise to different semantic systems; most results in the next section will be proven for an arbitrary choice of $\Gamma$. However, in this article, we mainly focus on the cases $\Gamma=\omega$ and $\Gamma=2^{\kappa}$, where $\kappa$ is the cardinality of the model. We will prove later that time limit bounds greater than $2^{\kappa}$ are not needed and that, in finite models, time limit bounds of the size $\kappa$ suffice.

Definition 3.10. Let $\mathcal{M}$ be a CGM, $q \in$ St and $\varphi$ an ATL formula. Let $\kappa$ be the cardinality of the model $\mathcal{M}$. We define three different notions of truth of $\varphi$ in $\mathcal{M}$ and $q$ based on the three different evaluation games, thereby defining the unbounded, bounded, and finitely bounded semantics, denoted by $\left|={ }_{u}^{g},\right|={ }_{b}^{g}$, and $\mid=_{f}^{g}$, respectively, as follows.

$-\mathcal{M}, q \models_{u}^{g} \varphi$ iff $\mathbf{E}$ has a winning strategy in $\mathcal{G}(\mathcal{M}, q, \varphi)$.

$-\mathcal{M}, q \models_{b}^{g} \varphi$ iff $\mathbf{E}$ has a winning strategy in $\mathcal{G}\left(\mathcal{M}, q, \varphi, 2^{\kappa}\right)$.

$-\mathcal{M}, q \models_{f}^{g} \varphi$ iff $\mathbf{E}$ has a winning strategy in $\mathcal{G}(\mathcal{M}, q, \varphi, \omega)$.

We also write more generally that $\mathcal{M}, q \mid=_{\Gamma}^{g} \varphi$ iff $\mathbf{E}$ has a winning strategy in $\mathcal{G}(\mathcal{M}, q, \varphi, \Gamma)$. This is called $\Gamma$-bounded semantics.

Note that, in the bounded GTS, the time limit bound $\Gamma$ is fixed based on the given model. On the other hand, $\Gamma$-bounded GTS has a fixed value of $\Gamma$ that is used for every model (this kind of semantics may also be called uniformly bounded).

We will prove that both the bounded and the unbounded semantics are equivalent to the standard compositional semantics of Definition 2.3. The finitely bounded semantics, on the other hand, will turn out nonequivalent to these but equivalent to a natural variant of the compositional semantics, introduced in Section 5. We will also see that each $\Gamma$-bounded GTS (for an arbitrary fixed value of $\Gamma$ ) is nonequivalent to the standard compositional semantics. The following example shows that the finitely bounded GTS differs from both the unbounded and bounded cases. In particular, the fixed point characterization of the temporal operator $\mathrm{F}$, that is, $\langle\langle A\rangle\rangle \mathrm{F} p \equiv p \vee\langle\langle A\rangle \mathrm{X}\langle\langle A\rangle\rangle \mathrm{F} p$, fails with finitely bounded GTS, as seen by the example.

Example 3.11. Consider the CGM $\mathcal{M}=\left(\{a\},\left\{q_{0}\right\} \cup \mathbb{N} \times \mathbb{N},\{p\}, \mathbb{N}, d, o, v\right)$ in Figure 1, where $v(p)=\{(i, i) \mid i \in \mathbb{N}\}, d\left(a, q_{0}\right)=\mathbb{N}, d(a,(i, j))=\{0\}, o\left(q_{0}, i\right)=(i, 0)$, and $o((i, j), 0)=(i, j+1)$.

In this model, $\mathcal{M},\left.q_{0}\right|_{f} ^{g}\langle\langle\emptyset\rangle \mathrm{F} p$ because, for every time limit $n<\omega$ chosen by Eloise, Abelard may select the action $n$ in the first round for the agent $a$. Thus, it will take $n+1$ rounds to reach a state in which $p$ is true. On the other hand, $\mathcal{M}, q_{0} \mid{ }^{g}{ }_{f}^{g}\langle\langle\emptyset\rangle\rangle\left\langle\langle\emptyset\rangle \mathrm{F} p\right.$; therefore, $\left.\mathcal{M}, q_{0}\right|{ }_{f}^{g} p \vee$ $\langle\langle\emptyset\rangle \mathrm{X}\langle\langle\emptyset\rangle \mathrm{F} p$ because after the first step, the game will be at a state $(i, 0)$ for some $i \in \mathbb{N}$, whence Eloise can choose any time limit $n \geq i$ and reach a state in which $p$ is true before time runs out.

However, $\mathcal{M},\left.q_{0}\right|_{{ }_{b}} ^{g}\langle\langle\emptyset\rangle \mathrm{F} p$, since Eloise can choose $\omega$ as the time limit in the beginning of the game and then lower it to $i<\omega$ when the next state $(i, 0)$ is reached. Also, $\mathcal{M},\left.q_{0}\right|_{{ }_{u}} ^{g}\langle\langle\emptyset\rangle \mathrm{F} p$ since a state where $p$ is true will always be reached in a finite number of steps.

Despite these observations, we will show that the three semantics become equivalent over image-finite models.

\section{ANALYZING EMBEDDED GAMES}

In this section, we will examine the properties of different versions of embedded games that occur as parts of evaluation games. We associate with each state a winning time label that describes how good that state is for the players. The optimal labels will be used to define a canonical strategy that 


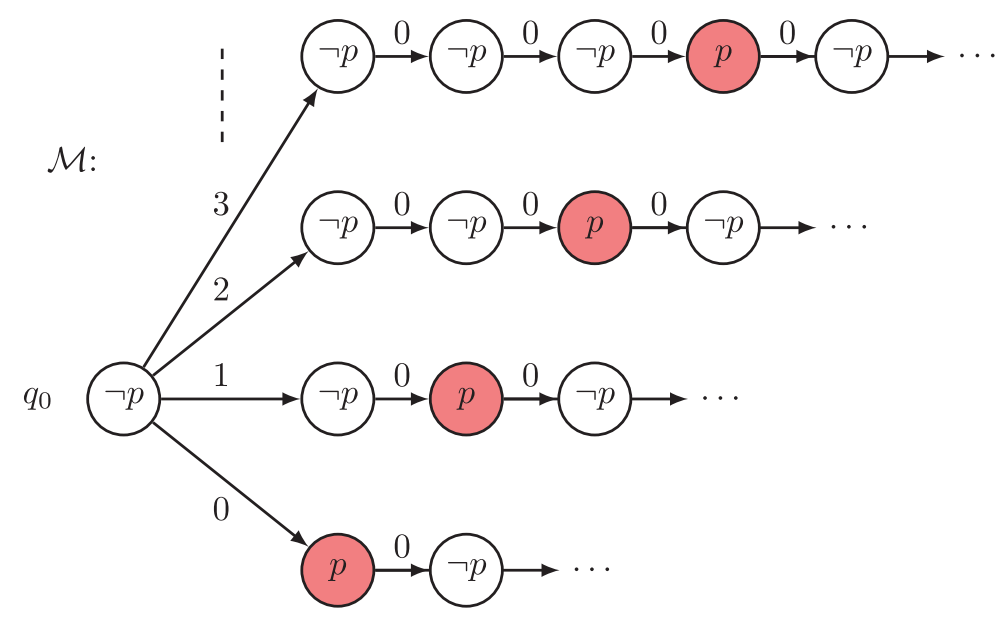

Fig. 1. $\langle\langle A\rangle \mathrm{F} p \equiv p \vee\langle\langle A\rangle \mathrm{X}\langle\langle A\rangle\rangle \mathrm{F} p$ fails in the finitely bounded GTS.

will be a winning strategy whenever one exists. With these notions, we shall prove positional determinacy of the embedded games. We will also show that if the players are allowed to announce sufficiently large ordinals as time limits, then bounded embedded games become essentially equivalent to corresponding unbounded embedded games. Furthermore, we shall analyze how the sizes of the needed ordinals depend on the CGM in which the game is played.

\subsection{Winning Time Labels}

Different values of the time limit bound $\Gamma$ correspond to different classes of bounded embedded games $G\left[\gamma_{0}\right]$, where $\gamma_{0}<\Gamma$. In this section, we use a fixed (unless otherwise specified) value of $\Gamma$ and will assume that all bounded embedded games are part of some evaluation game $\mathcal{G}\left(\mathcal{M}, q_{i n}, \varphi, \Gamma\right)$. Since $\Gamma$ could have any ordinal value, our results will hold, in particular, for both $2^{\kappa}$-bounded and finitely bounded semantics.

If $\mathrm{G}=\mathbf{g}\left(\mathbf{V}, \mathbf{C}, A, q_{0}, \psi_{\mathbf{C}}, \psi_{\overline{\mathrm{C}}}\right)$ is an embedded game and $q \in \mathrm{St}$, we write $\mathrm{G}[q]:=\mathbf{g}(\mathbf{V}, \mathbf{C}, A$, $\left.q, \psi_{\mathrm{C}}, \psi_{\overline{\mathrm{C}}}\right)$. We also use the abbreviation $\mathrm{G}[q, \gamma]:=(\mathrm{G}[q])[\gamma]$. This notation is useful because, by the recursive nature of bounded embedded games, any configuration $(\gamma, q)$ of $\mathrm{G}\left[\gamma_{0}\right]$ (where $\gamma_{0}<\Gamma$ ) is the initial configuration of $\mathrm{G}[q, \gamma]$.

We next define winning strategies for embedded games. Here, "winning an embedded game" intuitively means having a winning strategy in the evaluation game that continues from the exit position of the embedded game.

Definition 4.1. Let $\mathrm{G}=\mathrm{g}\left(\mathrm{V}, \mathrm{C}, A, q_{0}, \psi_{\mathrm{C}}, \psi_{\overline{\mathrm{C}}}\right)$ be an embedded game.

(1) We say that $\sigma_{\mathbf{P}}$ is a winning strategy for the player $\mathbf{P}$ in $\mathbf{G}$ if the following conditions hold:

(a) infinite plays are possible with $\sigma_{\mathbf{P}}$ only if $\mathbf{P} \neq \mathbf{C}$.

(b) the equivalence $\mathcal{M}, q \mid{ }^{g}{ }_{u}^{g} \psi \Leftrightarrow \mathbf{P}=\mathbf{V}$ holds for all exit positions $(\mathrm{V}, q, \psi)$ of $\mathrm{G}$ that can be reached when $\mathbf{P}$ plays using $\sigma_{\mathbf{P}}$.

(2) Let $\gamma_{0}<\Gamma$.

-Suppose that $\mathbf{P}=\mathbf{C}$. We say that the pair $\left(\sigma_{\mathrm{P}}, t\right)$ is a timed winning strategy for $\mathbf{P}$ in $\mathrm{G}\left[\gamma_{0}\right]$ if the equivalence $\mathcal{M}, q \models_{\Gamma}^{g} \psi \Leftrightarrow \mathbf{P}=\mathbf{V}$ holds for all exit positions $(\mathrm{V}, q, \psi)$ that can be encountered when $\mathbf{P}$ plays using the strategy $\sigma_{\mathrm{P}}$ and the timer $t$. 
- Suppose that $\mathbf{P} \neq \mathbf{C}$. We say that $\sigma_{\mathrm{P}}$ is a winning strategy for $\mathrm{P}$ in $\mathrm{G}\left[\gamma_{0}\right]$ if the equivalence $\mathcal{M}, q \mid \models_{\Gamma}^{g} \psi \Leftrightarrow \mathbf{P}=\mathbf{V}$ holds for all exit positions $(\mathbf{V}, q, \psi)$ that can occur when $\mathbf{P}$ plays using $\sigma_{\mathbf{P}}$.

If the unbounded (resp., bounded) embedded game ends in a position where the equivalence $\mathcal{M}, q \mid{ }^{g}{ }_{u} \psi \Leftrightarrow \mathbf{P}=\mathbf{V}$ (resp., $\mathcal{M}, q \mid=_{\Gamma}^{g} \psi \Leftrightarrow \mathbf{P}=\mathbf{V}$ ) holds, we say that $\mathbf{P}$ wins the embedded game. In the unbounded case, $\overline{\mathrm{C}}$ also wins in the case where the play is infinite. (Note that it now follows that, in every play of the embedded game, exactly one of the players wins.)

We next define for an embedded game $\mathrm{G}=\mathbf{g}\left(\mathbf{V}, \mathbf{C}, A, q_{0}, \psi_{\mathbf{C}}, \psi_{\overline{\mathrm{C}}}\right)$ so-called winning time labels, $\mathcal{L}_{\mathbf{P}}(q)$, for each $q \in$ St. The labels will indicate how good the state $q$ is for the player $\mathbf{P}$ when different bounded embedded games G $\left[q, \gamma_{0}\right]$ are played with different time limits $\gamma_{0}<\Gamma$. If the label is "win" or "lose," then the state is a winning (resp., losing) state for P, regardless of the time limit $\gamma_{0}$. If the label is an ordinal $\gamma<\Gamma$, it means that $\gamma$ is the "critical time limit" for winning or losing the game. More precisely, if $\mathbf{P}=\mathbf{C}, \gamma$ is the least time limit needed for $\mathbf{P}$ to win from $q$, and if $\mathbf{P} \neq \mathbf{C}$, then $\gamma$ is the least time limit such that $\mathbf{P}$ can no longer guarantee that he or she will not lose the game from $q$.

From now on, we will often consider separately the cases in which the player $\mathbf{P}$ is the controlling player $\mathrm{C}$ and where her or his opponent $\overline{\mathrm{P}}$ is the controlling player. The former case corresponds to the situation in which $\mathbf{P}$ is the verifier in an eventuality game or the falsifier in a safety game. The latter case means that $\mathbf{P}$ is the verifier in a safety game or is the falsifier in an eventuality game.

Definition 4.2. Let $\mathrm{G}=\mathbf{g}\left(\mathbf{V}, \mathrm{C}, A, q_{0}, \psi_{\mathrm{C}}, \psi_{\overline{\mathrm{C}}}\right)$ be an embedded game and $\mathbf{P} \in\{\mathrm{A}, \mathrm{E}\}$. The winning time label $\mathcal{L}_{\mathrm{P}}(q)$ for $\mathrm{P}$ in $\mathrm{G}$ at state $q \in \mathrm{St}$ is defined as follows.

Case 1. Suppose that $\mathbf{P}=\mathrm{C}$. Let $\sigma_{\mathrm{P}}$ be a strategy for $\mathrm{P}$. We first define a strategy label $l\left(q, \sigma_{\mathrm{P}}\right)$ as follows.

-Set $l\left(q, \sigma_{\mathrm{P}}\right):=$ lose if $\left(\sigma_{\mathrm{P}}, t\right)$ is not a timed winning strategy in $\mathrm{G}[q, \gamma]$ for any timer $t$ and $\gamma<\Gamma$.

-Else, set $l\left(q, \sigma_{\mathrm{P}}\right):=\gamma$, where $\gamma<\Gamma$ is the least time limit for which there is a timer $\mathrm{t}$ such that $\left(\sigma_{\mathrm{P}}, t\right)$ is a timed winning strategy in $\mathrm{G}[q, \gamma]$.

When there exists at least one strategy $\sigma_{\mathrm{P}}$ for $\mathrm{P}$ such that $l\left(q, \sigma_{\mathrm{P}}\right) \neq$ lose, we define $\mathcal{L}_{\mathbf{P}}(q)$ as the least ordinal value of strategy labels $l\left(q, \sigma_{\mathrm{P}}\right)$. Else, we define $\mathcal{L}_{\mathrm{P}}(q):=$ lose.

Case 2. Suppose that $\mathbf{P} \neq \mathbf{C}$. Let $\sigma_{\mathrm{P}}$ be a strategy for $\mathbf{P}$. We define $l\left(q, \sigma_{\mathrm{P}}\right)$ as follows.

-Set $l\left(q, \sigma_{\mathrm{P}}\right):=$ win if $\sigma_{\mathrm{P}}$ is a timed winning strategy in $\mathrm{G}[q, \gamma]$ for every time limit $\gamma<\Gamma$.

-Else, set $l\left(q, \sigma_{\mathrm{P}}\right):=\gamma$, where $\gamma<\Gamma$ is the least time limit such that $\sigma_{\mathrm{P}}$ is not a winning strategy in $\mathrm{G}[q, \gamma]$.

If $l\left(q, \sigma_{\mathrm{P}}\right)=$ win for some $\sigma_{\mathrm{P}}$, then set $\mathcal{L}_{\mathrm{P}}(q):=$ win. Else, set $\mathcal{L}_{\mathrm{P}}(q)$ to be the least upper bound for the values $l\left(q, \sigma_{\mathrm{P}}\right)$.

The following lemma shows that if the controller has a timed winning strategy within some time limit, then the controller has a timed winning strategy that is winning for all greater time limits as well. This claim may seem obvious but needs to be proven nevertheless, since there is no guarantee that a winning strategy for some smaller time limit would also make good choices at configurations with larger time limits. 


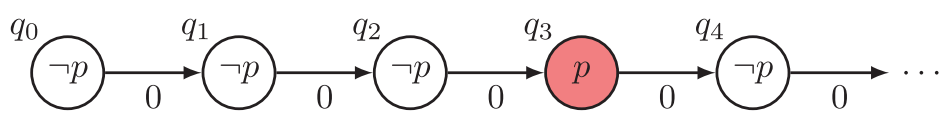

Fig. 2. An example of a game in which increasing the time limit bound lowers some winning time labels.

Lemma 4.3. Let $\mathbf{G}=\mathbf{g}\left(\mathrm{V}, \mathrm{C}, A, q_{0}, \psi_{\mathrm{C}}, \psi_{\overline{\mathrm{C}}}\right)$ be an embedded game. Assume that $\mathbf{P}=\mathbf{C}$ and that $\mathbf{P}$ has a timed winning strategy $\left(\sigma_{\mathrm{P}}, t\right)$ in $\mathrm{G}\left[\gamma_{0}\right]$ for some $\gamma_{0}<\Gamma$. Then, there is a pair $\left(\sigma_{\mathrm{P}}^{\prime}, t^{\prime}\right)$ that is a timed winning strategy in $\mathrm{G}[\gamma]$ for any time limit $\gamma$ such that $\gamma_{0} \leq \gamma<\Gamma$.

Proof. The idea here is that we define the timed strategy $\left(\sigma_{\mathrm{p}}^{\prime}, t^{\prime}\right)$ at a configuration $(\gamma, q)$ by using the choices given by $\left(\sigma_{\mathrm{P}}, t\right)$ for the smallest $\gamma^{\prime} \leq \gamma$ such that $\left(\sigma_{\mathrm{P}}, t\right)$ is a winning strategy for $\mathrm{G}\left[q, \gamma^{\prime}\right]$. See the appendix for a detailed proof.

The following proposition relates values of winning time labels to durations of embedded games and existence of winning strategies.

Proposition 4.4. Let $\mathrm{G}=\mathrm{g}\left(\mathrm{V}, \mathrm{C}, A, q_{0}, \psi_{\mathrm{C}}, \psi_{\overline{\mathrm{C}}}\right)$ be an embedded game, $\mathrm{P} \in\{\mathrm{A}, \mathrm{E}\}$ and $q \in \mathrm{St}$.

(1) If $\mathbf{P}=\mathrm{C}$, then:

(i) $\mathcal{L}_{\mathrm{P}}(q)=\gamma<\Gamma$ iff there is a pair $\left(\sigma_{\mathrm{P}}, t\right)$ that is a timed winning strategy in $\mathrm{G}\left[q, \gamma^{\prime}\right]$ for all $\gamma^{\prime}$ such that $\gamma \leq \gamma^{\prime}<\Gamma$, but there is no timed winning strategy for $\mathrm{P}$ in $\mathrm{G}\left[q, \gamma^{\prime}\right]$ for any $\gamma^{\prime}<\gamma$.

(ii) $\mathcal{L}_{\mathrm{P}}(q)=$ lose iff there is no timed winning strategy $\left(\sigma_{\mathrm{P}}, t\right)$ for $\mathrm{P}$ in $\mathrm{G}[q, \gamma]$ for any $\gamma<\Gamma$.

(2) If $\mathbf{P} \neq \mathbf{C}$, then:

(i) $\mathcal{L}_{\mathbf{P}}(q)=\gamma<\Gamma$ iff for every $\gamma^{\prime}<\gamma$, there is some $\sigma_{\mathrm{P}}$ that is a winning strategy for $\mathbf{P}$ in $\mathrm{G}\left[q, \gamma^{\prime}\right]$, but there is no winning strategy for $\mathrm{P}$ in $\mathrm{G}\left[q, \gamma^{\prime}\right]$ for any $\gamma^{\prime}$ such that $\gamma \leq \gamma^{\prime}<$ $\Gamma$.

(ii) $\mathcal{L}_{\mathrm{P}}(q)=$ win iff there is a strategy $\sigma_{\mathrm{P}}$ that is a winning strategy in $\mathrm{G}[q, \gamma]$ for every $\gamma<\Gamma$.

Proof. The proof follows quite directly from the definition of winning time labels. We also need to use Lemma 4.3 when proving case (1), (i). More details are given in the appendix.

Winning time labels $\mathcal{L}_{\mathrm{P}}(q)$ of an embedded game are either ordinals less than the time limit bound $\Gamma$, or else, labels win, lose. If we increased the value of $\Gamma$ to some $\Gamma^{\prime}>\Gamma$ and considered the values of winning time labels of the corresponding embedded game within the evaluation game $\mathcal{G}\left(\mathcal{M}, q_{i n}, \varphi, \Gamma^{\prime}\right)$, then some of the labels that originally were win or lose could now obtain ordinal values $\gamma$ such that $\Gamma \leq \gamma<\Gamma^{\prime}$. Other kinds of changes of labels would also be possible because the "truth sets" of the goal formulae $\psi_{\mathrm{C}}$ and $\psi_{\overline{\mathrm{C}}}$ could change; see the following example.

Example 4.5. Consider the CGM $\mathcal{M}=\left(\{a\},\left\{q_{i} \mid i \in \mathbb{N}\right\},\{p\},\{0\}, d, o, v\right)$, where $d\left(q_{i}\right)=\{0\}$ for each $i, o\left(q_{i}, 0\right)=q_{i+1}$ for each $i \in \mathbb{N}$ and $v(p)=\left\{q_{3}\right\}$ (see Figure 2). Let

$$
\psi:=\langle\langle\emptyset\rangle \mathrm{F} p .
$$

We consider the embedded game related to $\psi$ with the time limit bounds 3 and 4 . When $\Gamma=3$, Eloise has the following winning time labels: the state $q_{0}$ has the label lose, $q_{1}$ the label $2, q_{2}$ the label $1, q_{3}$ the label 0 , and all the other states have the label lose. When $\Gamma$ is increased to 4 , then the label of $q_{0}$ changes from lose to the value 3 , but the other labels remain the same.

Let then

$$
\varphi:=\langle\langle\emptyset\rangle \mathrm{F} \psi \quad(=\langle\langle\emptyset\rangle \mathrm{F}\langle\langle\emptyset\rangle \mathrm{F} p) .
$$


We then consider the embedded game related to $\varphi$ with the time limit bounds 3 and 4 . When $\Gamma=3$, Eloise's winning time labels are as follows: the state $q_{0}$ has the label 1 , the states $q_{1}, q_{2}$, and $q_{3}$ have the label 0 , and all the other states have the label lose. When $\Gamma$ is increased to 4 , then the label of $q_{0}$ is lowered from 1 to 0 , but all the other labels remain the same.

However, if all ordinal valued labels stay strictly below $\Gamma$ in all embedded games when going from $\Gamma$ to $\Gamma^{\prime}$, then each label, in fact, remains the same in the transition. This is shown by the following proposition.

Proposition 4.6. Let $\Gamma, \Gamma^{\prime}>0$ be ordinals such that $\Gamma<\Gamma^{\prime}$. Consider bounded evaluation games $\mathcal{G}_{\Gamma}=\mathcal{G}\left(\mathcal{M}, q_{i n}, \varphi, \Gamma\right)$ and $\mathcal{G}_{\Gamma^{\prime}}=\mathcal{G}\left(\mathcal{M}, q_{i n}, \varphi, \Gamma^{\prime}\right)$. Suppose that all the ordinal valued winning time labels for the embedded games of $\mathcal{G}_{\Gamma^{\prime}}$ are strictly smaller than $\Gamma$. Then, players have exactly the same winning time labels for the embedded games of $\mathcal{G}_{\Gamma^{\prime}}$ as they have for the embedded games of $\mathcal{G}_{\Gamma}$.

Proof (Sкетсн). Since winning time labels determine the time limits needed for winning, there is no need for the players to announce any higher ordinals than the winning time labels. Therefore, by the assumption that all winning time labels of the embedded games within $\mathcal{G}_{\Gamma^{\prime}}$ are below $\Gamma$, we can show that $\Gamma^{\prime}$-bounded GTS becomes equivalent with $\Gamma$-bounded GTS. From this it follows that, for any embedded game, the same winning time labels are constructed with respect to both $\Gamma$ and $\Gamma^{\prime}$. For more details, see the proof in the appendix.

We say that an ordinal $\Gamma$ is stable for an embedded game $G$ if the winning time labels of $G$ cannot be altered by increasing the time limit bound from $\Gamma$ to any higher ordinal. We say that $\Gamma$ is globally stable for a CGM $\mathcal{M}$ if $\Gamma$ is stable for all bounded embedded games within all evaluation games $\mathcal{G}\left(\mathcal{M}, q_{i n}, \varphi, \Gamma\right)$. By Proposition 4.6 , it is equivalent to say that $\Gamma$ is globally stable for $\mathcal{M}$ if, in the evaluation games for $\mathcal{M}$, we cannot create any labels with the ordinal value $\gamma \geq \Gamma$ by increasing the value of the time limit bound $\Gamma$ to any $\Gamma^{\prime}>\Gamma$.

We will see later that there exists a globally stable time limit bound for every concurrent game model. When $\Gamma$ is globally stable, its role is no longer as relevant since players would not benefit from the ability to choose arbitrarily high time limits. However, we always need some time limit bound in order to avoid strategies becoming proper classes.

\subsection{Canonical Strategies for Embedded Games}

Here, we define so-called canonical strategies. They are guaranteed to be winning strategies whenever a winning strategy exists. In the following definition, we first consider the case in which the player $\mathbf{P}$ is the controller $\mathbf{C}$.

Definition 4.7. Let $\mathrm{G}=\mathbf{g}\left(\mathbf{V}, \mathbf{C}, A, q_{0}, \psi_{\mathbf{C}}, \psi_{\overline{\mathrm{C}}}\right)$ be an embedded game, let $\mathbf{P} \in\{\mathbf{A}, \mathbf{E}\}$, and assume that $\mathbf{P}=\mathrm{C}$. We define a canonical strategy $\tau_{\mathrm{P}}$ for $\mathbf{P}$ in $\mathrm{G}$ for each $q \in \mathrm{St}$, as follows.

-If $\mathcal{L}_{\mathrm{P}}(q)=\gamma$, then $\tau_{\mathrm{P}}(q)=\sigma_{\mathrm{P}}(\gamma, q)$ for some strategy $\sigma_{\mathrm{P}}$ for which there is a timer $t$ such that $\left(\sigma_{\mathrm{P}}, t\right)$ is a timed winning strategy in $\mathrm{G}\left[q, \gamma^{\prime}\right]$ for all $\gamma^{\prime}$ such that $\gamma \leq \gamma^{\prime}<\Gamma$. (Note that such a strategy exists by Proposition 4.4.)

-If $\mathcal{L}_{\mathrm{P}}(q)=$ lose, then we put $\tau_{\mathrm{P}}(q)=$ any.

We define the canonical timer $\boldsymbol{t}_{\text {can }}$ for $\mathrm{P}$ in $\mathrm{G}$ for any pair $(\gamma, q)$ (where $\gamma<\Gamma$ is a limit ordinal and $q \in \mathrm{St}$ ) as follows.

-if $\mathcal{L}_{\mathrm{P}}(q) \neq$ lose and $\mathcal{L}_{\mathrm{P}}(q)<\gamma$, then $t_{\text {can }}(\gamma, q)=\mathcal{L}_{\mathrm{P}}(q) ;$

- otherwise, $t_{\text {can }}(\gamma, q)=$ any.

We call the pair $\left(\tau_{\mathrm{P}}, t_{c a n}\right)$ a canonically timed strategy (for the controller). 
Note that $\tau_{\mathrm{P}}$ is not necessarily unique since we may have to choose one from several strategies. However, these choices are all equally good for our purposes. Note also that the canonical strategy $\tau_{\mathrm{P}}$ depends only on states and can thus be used in both unbounded and bounded embedded games.

We will see that if $\mathrm{C}$ has a timed winning strategy in $\mathrm{G}\left[\gamma_{0}\right]$ for some $\gamma_{0}<\Gamma$, then $\mathrm{C}$ wins $\mathrm{G}\left[\gamma_{0}\right]$ with $\left(\tau_{\mathrm{C}}, t_{c a n}\right)$. The canonical strategy of $\mathrm{C}$ can also be seen as optimal for winning the game as fast as possible. This is because the canonical strategy always follows actions given by a strategy that is a winning strategy with the lowest possible time limit.

In the next definition, we consider different types of canonical strategies for the case in which the player $\mathbf{P}$ is not the controller $\mathbf{C}$.

Definition 4.8. Let $\mathrm{G}=\mathbf{g}\left(\mathbf{V}, \mathbf{C}, A, q_{0}, \psi_{\mathbf{C}}, \psi_{\overline{\mathrm{C}}}\right)$ be an embedded game, let $\mathbf{P} \in\{\mathbf{A}, \mathbf{E}\}$, and assume that $\mathbf{P} \neq \mathbf{C}$. We define a canonical strategy $\tau_{\mathbf{P}}$ for $\mathbf{P}$ in $\mathrm{G}\left[\gamma_{0}\right]$ for all $\gamma_{0}<\Gamma$ at every configuration $(\gamma, q)$, where $\gamma<\Gamma$ and $q \in \mathrm{St}$, as follows.

-If $\mathcal{L}_{\mathrm{P}}(q)=$ win, then $\tau_{\mathrm{P}}(\gamma, q)=\sigma_{\mathrm{P}}(\gamma, q)$ for some $\sigma_{\mathrm{P}}$ that is a winning strategy in $\mathrm{G}[q, \gamma]$ for every $\gamma<\Gamma$ (such a strategy exists by Proposition 4.4).

-Else, if $\mathcal{L}_{\mathbf{P}}(q)=\gamma^{\prime}$ and $\gamma^{\prime}>\gamma$, then $\tau_{\mathrm{P}}(\gamma, q)=\sigma_{\mathrm{P}}(\gamma, q)$ for some $\sigma_{\mathrm{P}}$ that is a winning strategy in $\mathrm{G}[q, \gamma]$ (such a strategy exists by Proposition 4.4).

-Otherwise, we define $\tau_{\mathbf{P}}(\gamma, q)=$ any.

We also define, for every $n<\omega$, the $\boldsymbol{n}$-canonical strategy $\tau_{\mathbf{P}}^{n}$ for $\mathbf{P}$ in $\mathbf{G}$ for each $q \in \mathrm{St}$, as follows.

-If $\mathcal{L}_{\mathrm{P}}(q) \geq \omega$ or $\mathcal{L}_{\mathrm{P}}(q)=$ win, then $\tau_{\mathrm{P}}^{n}(q)=\tau_{\mathrm{P}}(n, q)$.

-Else, if $\mathcal{L}_{\mathrm{P}}(q)=m>0$, then $\tau_{\mathrm{P}}^{n}(q)=\sigma_{\mathrm{P}}(m-1, q)$ for some $\sigma_{\mathrm{P}}$ that is a winning strategy in $\mathrm{G}[q, m-1]$. (Such a strategy exists by Proposition 4.4.)

-Otherwise, $\tau_{\mathbf{P}}^{n}(q)=$ any.

Finally, when $\Gamma$ is a successor ordinal, we define the $\infty$-canonical strategy $\tau_{\mathrm{P}}^{\infty}$ for $\mathrm{P}$ in $\mathrm{G}$ for each $q \in \mathrm{St}$, as follows.

-If $\mathcal{L}_{\mathrm{P}}(q)=$ win, then $\tau_{\mathrm{P}}^{\infty}(q)=\tau_{\mathrm{P}}(\Gamma-1, q)$.

-Otherwise, $\tau_{\mathrm{P}}^{\infty}(q)=$ any.

When $\mathbf{P} \neq \mathbf{C}$, the canonical strategy $\tau_{\mathbf{P}}$ depends on time limits; thus, it cannot be used in unbounded embedded games. However, both $n$-canonical and $\infty$-canonical strategies depend only on states. We fix the notation such that hereafter $\tau_{\mathrm{P}}, \tau_{\mathrm{P}}^{n}$, and $\tau_{\mathrm{P}}^{\infty}$ will always denote canonical strategies (of the respective type) for the player $\mathbf{P}$.

As we will see, canonical strategies $\tau_{\overline{\mathrm{C}}}$ for $\overline{\mathrm{C}}$ are optimal in a sense that, if $\overline{\mathrm{C}}$ has a winning strategy in $\mathrm{G}\left[\gamma_{0}\right]$ for some $\gamma_{0}<\Gamma$, then $\overline{\mathrm{C}}$ wins $\mathrm{G}\left[\gamma_{0}\right]$ with $\tau_{\overline{\mathrm{C}}}$. We will see later that $n$-canonical strategies are important in the finitely bounded evaluation games and $\infty$-canonical strategies in evaluation games with sufficiently large time limit bounds $\Gamma$. Intuitively, the $\infty$-canonical strategy always assumes that the highest possible time limit $\Gamma-1$ has been set for every position. Thus, when $\overline{\mathrm{C}}$ uses an $\infty$-canonical strategy, she or he plays as carefully as possible, always assuming the "worst" possible time limit $\Gamma-1$. The $n$-canonical strategy behaves in a similar way but under the assumption that the initial time limit $\gamma_{0}$ was set to at most $n$.

The following definition will be useful in our proofs later on.

Definition 4.9. Let $\mathrm{G}=\mathbf{g}\left(\mathbf{V}, \mathbf{C}, A, q_{0}, \psi_{\mathbf{C}}, \psi_{\overline{\mathrm{C}}}\right)$ be an embedded game. Let $\sigma_{\mathrm{P}}$ be a strategy in $\mathrm{G}\left[\gamma_{0}\right]$ $\left(\gamma_{0}<\Gamma\right)$. Consider a configuration $(\gamma, q)$ and suppose that $\sigma_{\mathrm{P}}(\gamma, q)$ is either a tuple of actions for $A$ or some response function for $\bar{A}$. We say that set $Q \subseteq$ St is forced by $\sigma_{\mathrm{P}}(\gamma, q)$ if, for each $q^{\prime} \in$ St, it holds that $q^{\prime} \in Q$ if and only if there is some play with $\sigma_{\mathrm{P}}$ from $(\gamma, q)$ such that the next 
configuration is $\left(\gamma^{\prime}, q^{\prime}\right)$ for some $\gamma^{\prime}$. We use the same terminology for the set forced by $\sigma_{\mathrm{P}}(q)$ when $\sigma_{\mathrm{P}}$ depends only on states.

The following proposition shows that the canonical strategy (with the canonical timer) is guaranteed to always be a (timed) winning strategy when such a strategy exists.

Proposition 4.10. Let $\mathrm{G}=\mathrm{g}\left(\mathrm{V}, \mathrm{C}, A, q_{0}, \psi_{\mathrm{C}}, \psi_{\overline{\mathrm{C}}}\right)$ be an embedded game, $\mathrm{P} \in\{\mathrm{A}, \mathrm{E}\}$, and $\gamma_{0}<\Gamma$.

(1) Suppose that $\mathbf{P}=\mathbf{C}$. If $\mathbf{P}$ has any timed winning strategy $\left(\sigma_{\mathrm{P}}, t\right)$ in $\mathrm{G}\left[\gamma_{0}\right]$, then $\left(\tau_{\mathrm{P}}, t_{\text {can }}\right)$ is a timed winning strategy for $\mathrm{P}$ in $\mathrm{G}\left[\gamma_{0}\right]$.

(2) Suppose that $\mathbf{P} \neq \mathbf{C}$. If $\mathbf{P}$ has any winning strategy $\sigma_{\mathrm{P}}$ in $\mathrm{G}\left[\gamma_{0}\right]$, then $\tau_{\mathrm{P}}$ is a winning strategy for $\mathbf{P}$ in $\mathrm{G}\left[\gamma_{0}\right]$.

Proof. The proof follows quite routinely from Definitions 4.7 and 4.8. See details in the appendix.

Recall Remark 3.9 on "genuinely positional" strategies. Since canonically timed strategies and canonical strategies depend on neither the initial state $q_{0}$ nor the initial time limit $\gamma_{0}$, it follows that if a player has a winning strategy in an embedded game, then the player has a genuinely positional winning strategy. The converse also clearly holds: if the player has a genuinely positional winning strategy, the player has a standard winning strategy.

By the first claim of the previous proposition, we see that it suffices to consider those strategies of player $\mathrm{C}$ that are independent of time limits in configurations. The following lemma shows that the same holds for the player $\overline{\mathrm{C}}$ in bounded embedded games with a finite time limit. The key here will be the use of $n$-canonical strategies that depend only on states.

Lemma 4.11. Let $\mathrm{G}=\mathbf{g}\left(\mathbf{V}, \mathrm{C}, A, q_{0}, \psi_{\mathrm{C}}, \psi_{\overline{\mathrm{C}}}\right)$ be an embedded game, let $\mathbf{P} \in\{\mathbf{A}, \mathbf{E}\}$, and assume that $\mathbf{P} \neq \mathbf{C}$. For any $n<\omega$, if $\mathbf{P}$ has a winning strategy $\sigma_{\mathbf{P}}$ in $\mathrm{G}[m]$ for some $m \leq n$, then $\tau_{\mathrm{P}}^{n}$ is a winning strategy in $\mathrm{G}[\mathrm{m}]$.

Proof. We will prove by induction on $m \leq n$ that, for any $q \in \mathrm{St}$, if $\mathbf{P}$ has a winning strategy in $\mathrm{G}[q, m]$, then $\tau_{\mathbf{P}}^{n}$ is a winning strategy in $\mathbf{G}[q, m]$. If $m=0$ and $\mathbf{P}$ has a winning strategy $\sigma_{\mathbf{P}}$ in $\mathrm{G}[q, 0]$, then every strategy of $\mathbf{P}$ will be a winning strategy in $\mathrm{G}[q, 0]$. Hence, in particular, $\tau_{\mathrm{P}}^{n}$ is a winning strategy in $\mathrm{G}[q, 0]$.

Suppose, then, that the claim holds for $m-1$ and that $\mathbf{P}$ has a winning strategy in $\mathrm{G}[q, m]$. Thus, by Proposition 4.4 , we have that $\mathcal{L}_{\mathrm{P}}(q)>m$ or $\mathcal{L}_{\mathrm{P}}(q)=$ win.

Suppose first that $\mathcal{L}_{\mathbf{P}}(q)=m^{\prime}<\omega$, and let $\sigma_{\mathrm{P}}$ be a strategy for which $l\left(q, \sigma_{\mathrm{P}}\right)=m^{\prime}$ and $\tau_{\mathrm{P}}^{n}(q)=$ $\sigma_{\mathrm{P}}\left(m^{\prime}-1, q\right)$ (such a strategy $\sigma_{\mathrm{P}}$ exists by the definition of $\left.\tau_{\mathrm{P}}^{n}\right)$. Let $Q \subseteq$ St be the set of states forced by $\sigma_{\mathrm{P}}\left(m^{\prime}-1, q\right)$. Since $m^{\prime}>m$, the strategy $\sigma_{\mathrm{P}}$ must be a winning strategy in $\mathrm{G}[q, m]$; thus, it will also be a winning strategy in $\mathrm{G}\left[q^{\prime}, m-1\right]$ for every $q^{\prime} \in Q$. Thus, by the inductive hypothesis, $\tau_{\mathrm{P}}^{n}$ is a winning strategy in $\mathrm{G}\left[q^{\prime}, m-1\right]$ for every $q^{\prime} \in Q$. Therefore, we observe that $\tau_{\mathrm{P}}^{n}$ will also be a winning strategy in $\mathrm{G}\left[q^{\prime}, m\right]$.

Suppose, then, that $\mathcal{L}_{\mathrm{P}}(q) \geq \omega$ or $\mathcal{L}_{\mathrm{P}}(q)=$ win, and let $\sigma_{\mathrm{P}}$ be a strategy such that $l\left(q, \sigma_{\mathrm{P}}\right) \in$ $\{$ win $\} \cup\{\gamma<\Gamma \mid \gamma>n\}$ and $\tau_{\mathrm{P}}^{n}(q)=\tau_{\mathrm{P}}(n, q)=\sigma_{\mathrm{P}}(n, q)$. (Recall Definition 4.8; the strategy $\sigma_{\mathrm{P}}$ exists by the definitions of $\tau_{\mathrm{P}}^{n}$ and $\tau_{\mathrm{P}}$.) Let $Q \subseteq$ St be the set of states that is forced by $\sigma_{\mathrm{P}}(n, q)$. Since $m \leq n$, the strategy $\sigma_{\mathrm{P}}$ is a winning strategy in $\mathrm{G}[q, m]$; thus, it is also a winning strategy in $\mathrm{G}\left[q^{\prime}, m-1\right]$ for every $q^{\prime} \in Q$. Hence, by the inductive hypothesis, $\tau_{\mathrm{P}}^{n}$ is a winning strategy in $\mathrm{G}\left[q^{\prime}, m-1\right]$ for every $q^{\prime} \in Q$. Thus, we observe that $\tau_{\mathrm{P}}^{n}$ is a winning strategy in $\mathrm{G}[q, m]$.

Example 4.12. If $\mathcal{L}_{\overline{\mathrm{C}}}(q)=\omega$, the player $\overline{\mathrm{C}}$ can win the game with any time limit $n<\omega$, but there is no single strategy that would win for every $n$. However, if $\overline{\mathrm{C}}$ knows that the initial time limit is (at most) $m$, then $\overline{\mathrm{C}}$ knows that the $m$-canonical strategy will a winning strategy. Therefore, 
intuitively, $\overline{\mathrm{C}}$ only needs to know the time limit when selecting the strategy, but not when using it (since $n$-canonical strategies depend on states only).

\subsection{Determinacy of Bounded Embedded Games}

The correspondence in the following proposition between the winning time labels of $\mathrm{C}$ and $\overline{\mathrm{C}}$ will be the key for proving determinacy of bounded embedded games. The main idea for proving the proposition is similar to the one in the standard proof of the Gale-Stewart Theorem.

Proposition 4.13. Let $\mathrm{G}=\mathbf{g}\left(\mathrm{V}, \mathrm{C}, A, q_{0}, \psi_{\mathrm{C}}, \psi_{\overline{\mathrm{C}}}\right)$ be an embedded game. Then, for each state $q \in \mathrm{St}$ and each ordinal $\gamma<\Gamma$, it holds that $\mathcal{L}_{\mathrm{C}}(q)=\gamma$ iff $\mathcal{L}_{\overline{\mathrm{C}}}(q)=\gamma$.

Proof (Sкетсн). We can prove the claim by transfinite induction on $\gamma$. The case $\gamma=0$ is clear, since if $\mathrm{C}$ cannot win the game with time limit 0 , then $\overline{\mathrm{C}}$ will win it automatically. We then suppose that the claim holds for every $\gamma^{\prime}<\gamma$ and prove the equivalence for $\gamma$. If $\mathcal{L}_{\mathrm{C}}(q)=\gamma$, then $\mathrm{C}$ has a winning strategy in $\mathrm{G}[q, \gamma]$ and, thus, $\overline{\mathrm{C}}$ cannot have a winning strategy in that game. Hence, by Proposition 4.4, we have that $\mathcal{L}_{\overline{\mathrm{C}}} \leq \gamma$. By the inductive hypothesis, $\mathcal{L}_{\overline{\mathrm{C}}} \neq \gamma^{\prime}$ for every $\gamma^{\prime}<\gamma$ and; thus, $\mathcal{L}_{\overline{\mathrm{C}}}(q)=\gamma$.

Suppose, then, that $\mathcal{L}_{\overline{\mathrm{C}}}(q)=\gamma$. If there existed some $\sigma_{\overline{\mathrm{C}}}, \gamma^{\prime}<\Gamma$ and $Q \subseteq$ St forced by $\sigma_{\overline{\mathrm{C}}}\left(\gamma^{\prime}, q\right)$ such that $\mathcal{L}_{\overline{\mathrm{C}}}\left(q^{\prime}\right) \geq \gamma$ for every $q^{\prime} \in Q$, then we could have used $\sigma_{\overline{\mathrm{C}}}$ to construct a winning strategy for $\overline{\mathrm{C}}$ in $\mathrm{G}[q, \gamma]$. This is not possible since we have that $\mathcal{L}_{\overline{\mathrm{C}}}(q)=\gamma$. Thus, it can be shown that $\mathrm{C}$ can play so that, for all possible successor states $q^{\prime}$, we have that $\mathcal{L}_{\overline{\mathrm{C}}}\left(q^{\prime}\right)<\gamma$, whence by the inductive hypothesis $\mathcal{L}_{\mathrm{C}}\left(q^{\prime}\right)<\gamma$. Hence, we can construct a timed winning strategy for $\mathrm{C}$ in $\mathrm{G}[q, \gamma]$ and thus infer that $\mathcal{L}_{\mathrm{C}}(q)=\gamma$. For a detailed proof, see the appendix.

Recall that, apart from ordinal values that are less than the bound $\Gamma$, the only possible winning time label for $\mathbf{C}$ is the label lose (see Definition 4.2). Similarly, for $\overline{\mathbf{C}}$, the only nonordinal value for the labels is win. Hence, by the previous proposition, we also have that

$$
\mathcal{L}_{\mathrm{C}}(q)=\text { lose iff } \mathcal{L}_{\overline{\mathrm{C}}}(q)=\text { win. }
$$

It is now easy to show that all bounded embedded games are (positionally) determined.

Corollary 4.14. C has a timed winning strategy in a bounded embedded game $\mathrm{g}\left(\mathrm{V}, \mathrm{C}, A, q_{0}\right.$, $\left.\psi_{\mathrm{C}}, \psi_{\overline{\mathrm{C}}}\right)\left[\gamma_{0}\right]$ iff $\overline{\mathrm{C}}$ does not have a winning strategy in that game.

Proof. If $\mathcal{L}_{\mathrm{C}}\left(q_{0}\right)=$ lose, then $\mathcal{L}_{\overline{\mathrm{C}}}\left(q_{0}\right)=$ win, from which by Proposition 4 .4, the player $\overline{\mathrm{C}}$ has a winning strategy and $\mathrm{C}$ does not have a timed winning strategy. Else $\mathcal{L}_{\mathrm{C}}\left(q_{0}\right)=\gamma$ for some $\gamma<\Gamma$. Now, by Proposition 4.13, also $\mathcal{L}_{\overline{\mathrm{C}}}\left(q_{0}\right)=\gamma$. If $\gamma \leq \gamma_{0}$, then, by Proposition 4.4, the player $\mathrm{C}$ has a timed winning strategy, while $\overline{\mathbf{C}}$ does not have a winning strategy. Analogously, if $\gamma>\gamma_{0}$, then $\bar{C}$ has a winning strategy, while $\mathrm{C}$ does not have a timed winning strategy.

Due to the positional determinacy of bounded embedded games, we can prove that bounded evaluation games are also positionally determined.

Proposition 4.15. Let $\mathcal{M}$ be a CGM, $q \in \mathrm{St}$, $\varphi$ an ATL-formula, $\Gamma>0$ an ordinal and $\mathbf{P} \in\{\mathrm{A}, \mathrm{E}\}$. Then, $\mathbf{P}$ has a winning strategy in $\mathcal{G}(\mathcal{M}, q, \varphi, \Gamma)$ if and only if $\overline{\mathbf{P}}$ does not have a winning strategy in $(\mathcal{G}, q, \varphi, \Gamma)$.

Proof (Sкетсн). Since embedded games are positionally determined, we can prove the positional determinacy of the evaluation games by a simple induction on $\varphi$. For a detailed proof, see the appendix.

Consequently, the following equivalence holds: $\mathcal{M}, q||_{\Gamma}^{g} \neg \varphi$ iff $\mathcal{M}, q \nvdash_{\Gamma}^{g} \varphi$. 


\subsection{Finding Stable Time Limit Bounds}

Definition 4.16. Let $\mathcal{M}$ be a CGM and let $q \in$ St. We define the branching degree of $\boldsymbol{q}, \operatorname{BD}(q)$ to be the cardinality of the set of states accessible from $q$ with a single transition:

$$
\operatorname{BD}(q):=\operatorname{card}(\{o(q, \vec{\alpha}) \mid \vec{\alpha} \in \operatorname{action}(\mathbb{A g t}, q)\}) .
$$

We define the regular branching bound of $\mathcal{M}$ to be the smallest cardinal $\operatorname{RBB}(\mathcal{M})$ for which the following conditions hold:

(1) $\operatorname{RBB}(\mathcal{M})>\operatorname{BD}(q)$ for every $q \in$ St.

(2) $\operatorname{RBB}(\mathcal{M})$ is infinite.

(3) $\operatorname{RBB}(\mathcal{M})$ is a regular cardinal.

We will see that the value of $\operatorname{RBB}(\mathcal{M})$ is always a globally stable time limit bound for $\mathcal{M}$. We will also see that the regular branching bound is, in a sense, the smallest possible time limit bound that is guaranteed to be stable (see the claim of Proposition 4.23).

Remark 4.17. Let $\kappa$ be the smallest infinite cardinal for which $\mathrm{BD}(q)<\kappa$ for every $q \in$ St. Since successor cardinals are always regular, we must have that $\operatorname{RBB}(\mathcal{M}) \leq \kappa^{+}$(here, $\kappa^{+}$is the successor cardinal of $\kappa$ ). However, in a typical case with infinite models, we have that $\operatorname{RBB}(\mathcal{M})=\kappa$. In particular,

$$
\operatorname{RBB}(\mathcal{M})=\omega \text { iff } \mathcal{M} \text { is image finite (i.e., } \operatorname{BD}(q)<\omega \text { for every } q \in \mathrm{St} \text { ). }
$$

Also, if $\operatorname{card}($ Act $) \leq \kappa$ or $\operatorname{card}(\mathrm{St}) \leq \kappa$ for an infinite $\kappa$, then, necessarily, $\operatorname{RBB}(\mathcal{M}) \leq \kappa^{+}$.

The following lemma shows an important correspondence between the canonical strategies and the winning time labels of the controller.

Lemma 4.18. Let $\mathrm{G}=\mathrm{g}\left(\mathrm{V}, \mathrm{C}, A, q_{0}, \psi_{\mathrm{C}}, \psi_{\overline{\mathrm{C}}}\right)$ be an embedded game, $\mathrm{P} \in\{\mathrm{A}, \mathrm{E}\}$ and $\mathbf{P}=\mathrm{C}$. The following holds for every $q \in \mathrm{St}$ : if $\mathcal{L}_{\mathrm{P}}(q)=\gamma>0$ and $Q \subseteq$ St is forced by $\tau_{\mathrm{P}}(q)$, then $\mathcal{L}_{\mathrm{P}}\left(q^{\prime}\right)<\gamma$ for every $q^{\prime} \in Q$, and

$-\max \left\{\mathcal{L}_{\mathrm{P}}\left(q^{\prime}\right) \mid q^{\prime} \in Q\right\}=\gamma-1$, if $\gamma$ is a successor ordinal, and

$-\sup \left\{\mathcal{L}_{\mathbf{P}}\left(q^{\prime}\right) \mid q^{\prime} \in Q\right\}=\gamma$, if $\gamma$ is a limit ordinal.

Proof (Sкetch). When $\mathcal{L}_{\mathrm{P}}(q)=\gamma>0$, by Proposition $4.10,\left(\tau_{\mathrm{P}}, t_{\text {can }}\right)$ is a timed winning strategy in $\mathrm{G}[q, \gamma]$. Therefore, every winning time label in the set $Q$ forced by $\tau_{\mathrm{P}}(q)$ must be an ordinal less than $\gamma$. If $\gamma$ is a successor ordinal, then there must be some state with label $\gamma-1$ in $Q$, and if $\gamma$ is a limit ordinal, then $\gamma$ must be the supremum of the labels in $Q$ (else, there would be a winning strategy for $\mathrm{C}$ in $\mathrm{G}\left[q, \gamma^{\prime}\right]$ for some $\gamma^{\prime}<\gamma$ ). For more details, see the proof in the appendix.

The following lemma shows that if a certain ordinal-valued winning time label exists for an embedded game, then all the smaller winning time labels must exist for that game, as well.

Lemma 4.19. Let $\mathrm{G}=\mathrm{g}\left(\mathrm{V}, \mathrm{C}, A, q_{0}, \psi_{\mathrm{C}}, \psi_{\overline{\mathrm{C}}}\right)$ be an embedded game and $\gamma<\Gamma$ an ordinal. Assume that $\mathcal{L}_{\mathbf{P}}(q)=\gamma$ for some $q \in \mathrm{St}$ and $\mathbf{P} \in\{\mathrm{A}, \mathrm{E}\}$. Then, for every $\delta \leq \gamma$, there is a state $q_{\delta}$ for which $\mathcal{L}_{\mathrm{C}}\left(q_{\delta}\right)=\delta$.

Proof. We prove the claim by transfinite induction on $\gamma<\Gamma$ for every $q \in$ St. Let the inductive hypothesis be that the claim holds for every $\gamma^{\prime}<\gamma$ and suppose that either of the players has winning time label $\gamma$ at some state $q$. By Proposition 4.13, we have that $\mathcal{L}_{\mathrm{C}}(q)=\gamma$.

Assume that $\delta<\gamma$. If $\gamma$ is a successor ordinal, then, by Lemma 4.18, there is a state $q^{\prime} \in \mathrm{St}$ such that $\mathcal{L}_{\mathrm{C}}\left(q^{\prime}\right)=\gamma-1$. Since $\delta \leq \gamma-1$, by the inductive hypothesis there is a state $q_{\delta}$ for which $\mathcal{L}_{\mathrm{C}}\left(q_{\delta}\right)=\delta$. Suppose, then, that $\gamma$ is a limit ordinal. By Lemma 4.18 , there must be a state $q^{\prime} \in \mathrm{St}$ 
such that $\mathcal{L}_{\mathrm{C}}\left(q^{\prime}\right)=\gamma^{\prime}$ for some ordinal $\gamma^{\prime}$ such that $\delta<\gamma^{\prime}<\gamma$. Hence, by the inductive hypothesis, there is a state $q_{\delta}$ for which $\mathcal{L}_{\mathrm{C}}\left(q_{\delta}\right)=\delta$.

In the following proposition, we show that in finite models, all winning time labels are strictly smaller than the cardinality of the model.

Proposition 4.20. Let $\mathcal{M}$ be a CGM. If $\operatorname{card}(\mathcal{M})=n<\omega$, then $n$ is a globally stable time limit bound for $\mathcal{M}$.

Proof. (Recall that, by Proposition 4.6, if all possible winning time labels are below an ordinal $\Gamma$ for an arbitrary time limit bound $\Gamma^{\prime}$, then $\Gamma$ is globally stable for $\mathcal{M}$.) If there was some state with a winning time label $\gamma \geq n$, then, by Lemma 4.19, there would be a state $q \in$ St for which $\mathcal{L}_{\mathrm{C}}(q)=$ $n$. Further, by Lemma 4.19, we would now find states with winning time labels $n-1, n-2, \ldots, 0$. But since each state may only have a single label, this would mean that $\operatorname{card}(\mathcal{M}) \geq n+1>n$, a contradiction. (This claim is also quite obvious by the observation that the controller can only win the embedded game by reaching a state in the truth set of the formula $\psi_{\mathrm{C}}$. Hence, it would not be beneficial for the controller to go in cycles.)

The following result shows how to find an upper bound for the values of possible winning time labels by just looking at the regular branching bound of a model. Recall that a cardinal $\kappa$ is regular if $\kappa$ is equal to its own cofinality, i.e., there is no set of less than $\kappa$ many ordinals, each less than $\kappa$, with supremum $\kappa$.

Proposition 4.21. Let $\mathcal{M}$ be a CGM. Then, $\operatorname{RBB}(\mathcal{M})$ is globally stable for $\mathcal{M}$.

Proof. For the sake of contradiction, suppose that there is $\Gamma^{\prime}>\operatorname{RBB}(\mathcal{M})$ and an embedded game $\mathrm{G}$ within a bounded evaluation game $\mathcal{G}\left(\mathcal{M}, q_{i n}, \varphi, \Gamma^{\prime}\right)$ such that in $\mathrm{G}$ at least one of the players has winning time labels that are greater than or equal to $\operatorname{RBB}(\mathcal{M})$. By Lemma 4.19, there is $q \in$ St for which $\mathcal{L}_{\mathrm{C}}(q)=\operatorname{RBB}(\mathcal{M})$. Let $Q \subseteq$ St be the set of states that is forced by $\tau_{\mathrm{C}}(q)$. Now, $\operatorname{card}(Q) \leq \mathrm{BD}(q)<\mathrm{RBB}(\mathcal{M})$. By Lemma $4.18, \mathcal{L}_{\mathrm{C}}\left(q^{\prime}\right)<\mathrm{RBB}(\mathcal{M})$ for every state $q^{\prime} \in Q$; furthermore, since $\operatorname{RBB}(\mathcal{M})$ is a limit ordinal, $\operatorname{RBB}(\mathcal{M})$ must be the supremum of the winning time labels of the states in $Q$, that is, $\sup \left\{\mathcal{L}_{\mathrm{C}}\left(q^{\prime}\right) \mid q^{\prime} \in Q\right\}=\operatorname{RBB}(\mathcal{M})$.

Now, every winning time label in $Q$ is smaller than $\operatorname{RBB}(\mathcal{M})$ and the cardinality of $Q$ is less than $\operatorname{RBB}(\mathcal{M})$. Because $\operatorname{RBB}(\mathcal{M})$ is a regular cardinal, it is equal to its own cofinality. Hence, we must have that $\sup \left\{\mathcal{L}_{\mathrm{C}}\left(q^{\prime}\right) \mid q^{\prime} \in Q\right\}<\operatorname{RBB}(\mathcal{M})$. This is a contradiction; thus, $\operatorname{RBB}(\mathcal{M})$ must be globally stable for $\mathcal{M}$ (recall Proposition 4.6 on global stability).

From Proposition 4.21, we obtain the following corollary on the stability of the time limit bounds used with the bounded and finitely bounded GTS.

Corollary 4.22. Let $\mathcal{M}$ be any CGM.

(1) If $\operatorname{card}(\mathcal{M})=\kappa$, then $2^{\kappa}$ is a globally stable time limit bound for $\mathcal{M}$.

(2) If $\mathcal{M}$ is image finite, then $\omega$ is a globally stable time limit bound for $\mathcal{M}$.

Proof. (1) Let $\operatorname{card}(\mathcal{M})=\kappa$. If $\kappa<\omega$, then, by Proposition $4.20, \kappa$ is globally stable for $\mathcal{M}$, whence also $2^{\kappa}$ is globally stable for $\mathcal{M}$. And, if $\kappa \geq \omega$, then $\operatorname{RBB}(\mathcal{M}) \leq \kappa^{+} \leq 2^{\kappa}$ (see Remark 4.17). Thus, by Proposition 4.21, $2^{\kappa}$ is a globally stable time limit bound for $\mathcal{M}$.

(2) If $\mathcal{M}$ is image finite, then $\operatorname{RBB}(\mathcal{M})=\omega$. By Proposition 4.21, $\omega$ is globally stable for $\mathcal{M}$.

Note that we could replace $2^{\kappa}$ with $\kappa^{+}$in case (1) above. This gives us a stronger result in the models of set theory that do not satisfy the generalized continuum hypothesis. Also, note that since $\operatorname{RBB}(\mathcal{M})$ is determined only by the size of the branchings in the model, the cardinality $\kappa$ of $\mathcal{M}$ might be much greater than $\operatorname{RBB}(\mathcal{M})$ (which is globally stable for $\mathcal{M}$ ). 
As seen by Proposition 4.18, only sufficiently large branchings can generate winning time labels of higher cardinality. Thus, when estimating the value of a stable time limit bound, it suffices to check the sizes of the large branchings in the model. We know by Proposition 4.21 that $\operatorname{RBB}(\mathcal{M})$ is guaranteed to be stable for a CGM $\mathcal{M}$, but can we give any better estimate of a stable bound by just looking at the branchings in $\mathcal{M}$ ? The answer is negative in the sense of the following Proposition 4.23. It implies that only knowing the least infinite strict upper bound of the branchings $\mathrm{BD}(q)$ of all states $q$ of a $\mathrm{CGM} \mathcal{M}$, no lower time limit bound than $\operatorname{RBB}(\mathcal{M})$ is guaranteed to be globally stable for $\mathcal{M}$. Before the proposition, we give some auxiliary definitions.

Let $\mathcal{M}$ be a CGM and let $\kappa$ be an infinite cardinal such that the branching degrees in $\mathcal{M}$ are strictly bounded by $\kappa$, that is, $\mathrm{BD}(q)<\kappa$ for every $q \in \mathrm{St}$. Then, we say that $\mathcal{M}$ is less than $\boldsymbol{\kappa}$ branching. For an ordinal $\gamma$, we say that a CGM $\mathcal{N}$ realizes $\gamma$ if there is some state of $\mathcal{N}$ that realizes the winning time label $\gamma$ in some embedded game.

Proposition 4.23. Let $\kappa$ be an infinite cardinal. Then, there exists a less than $\kappa$-branching model $\mathcal{M}$ that realizes every $\gamma<\operatorname{RBB}(\mathcal{M})$ and where all branchings strictly less than $\kappa$ occur.

Proof. We begin the proof by constructing by transfinite induction a model $\mathcal{M}_{\gamma}$ for each $\gamma$ such that $\mathcal{M}_{\gamma}$ realizes exactly all ordinals less than or equal to $\gamma$. Furthermore, the following conditions hold.

(1) If $\gamma$ is a finite ordinal, then the branching degree $\operatorname{BD}(q)$ of each state $q$ in $\mathcal{M}_{\gamma}$ is 1 .

(2) If $\gamma$ is an infinite ordinal, then $\operatorname{card}(\gamma) \leq \gamma$ is an upper bound for the branchings $\operatorname{BD}(q)$ of the states $q$ in $\mathcal{M}_{\gamma}$. Furthermore, the following conditions hold.

(a) If $\operatorname{card}(\gamma)$ is a regular cardinal, then $\mathcal{M}_{\gamma}$ has a node with branching $\operatorname{card}(\gamma)$.

(b) If card $(\gamma)$ is a singular (i.e., nonregular) cardinal, then all states in $\mathcal{M}_{\gamma}$ have branching strictly less than $\operatorname{card}(\gamma)$.

All such models $\mathcal{M}_{\gamma}$ will be of the form $\mathcal{M}_{\gamma}=(\mathbb{A g t}$, St, $\Pi$, Act, $d, o, v)$, where $\mathbb{A g t}=\{a\}, q_{0} \in \mathrm{St}$, $\Pi=\{p\}$, and Act $=\{\delta \mid \delta<\gamma\}$. We will always establish that the winning time label for Eloise at the state $q_{0}$ in the embedded game $\mathrm{G}=\left(\mathrm{E}, \mathrm{E}, \emptyset, q_{0}, p, \mathrm{~T}\right)$ (which arises when verifying the formula $\varphi=\left\langle\langle\emptyset\rangle \mathrm{F} p\right.$ at $\left.q_{0}\right)$ is equal to $\gamma$.

If $\gamma=0$, we define $\mathcal{M}_{0}=(\mathbb{A g t}, \mathrm{St}, \Pi$, Act, $d, o, v)$, where St $=\left\{q_{0}\right\}, d\left(q_{0}, a\right)=\{0\}, o\left(q_{0}, 0\right)=q_{0}$, and $v(p)=\left\{q_{0}\right\}$. Now, $\operatorname{BD}\left(q_{0}\right)=1$ and $\mathcal{L}_{\mathrm{E}}\left(q_{0}\right)=0$.

Suppose, then, that $\gamma$ is a successor ordinal. Consider the model $\mathcal{M}_{\gamma-1}$ with $\mathcal{L}_{\mathrm{E}}\left(q_{0}\right)=\gamma-1$ obtained by the induction hypothesis. Let $\mathcal{M}_{\gamma-1}^{\prime}=\left(\mathbb{A g t}, \mathrm{St}^{\prime}, \Pi\right.$, Act, $\left.d^{\prime}, o^{\prime}, v^{\prime}\right)$ be an isomorphic copy of $\mathcal{M}_{\gamma-1}$ in which the state $q_{0}$ is replaced by a new state $q^{\prime}$. Let $\mathcal{M}_{\gamma}=(\mathbb{A g t}, \mathrm{St}, \Pi$, Act, $d, o, v)$, where we define $\mathrm{St}:=\mathrm{St}^{\prime} \cup\left\{q_{0}\right\}, d:=d^{\prime} \cup\left\{\left(\left(q_{0}, a\right),\{0\}\right)\right\}, o:=o^{\prime} \cup\left\{\left(\left(q_{0}, 0\right), q^{\prime}\right)\right\}$, and $v:=v^{\prime}$. Since $\mathrm{BD}\left(q_{0}\right)=1$, the branchings of $\mathcal{M}_{\gamma}$ are bounded in the desired way by the induction hypothesis. It is easy to see that $\mathcal{L}_{\mathrm{E}}\left(q_{0}\right)=\gamma$, and all ordinals less than $\gamma$ are realized in $\mathcal{M}_{\gamma}$ by the induction hypothesis.

Suppose, then, that $\gamma$ is a limit ordinal. We next construct, for later use, a set of ordinals $\Psi \subseteq\{\delta \mid \delta<\gamma\}$ such that $\sup (\Psi)=\gamma$. If $\operatorname{card}(\gamma)$ is a regular cardinal, then we set $\Psi=\{\delta \mid \delta<\gamma\}$. Otherwise, when $\operatorname{card}(\gamma)$ is a singular cardinal, we do the following. Let $\mu$ be the cofinality of $\gamma$, whence there exists some set $S$ of ordinals strictly less than $\gamma \operatorname{such}$ that $\sup (S)=\gamma$ and $\operatorname{card}(S)=\mu$. Let $\Psi:=S$. Note that the following conditions hold.

(1) If $\operatorname{card}(\gamma)$ is regular, then $\operatorname{card}(\Psi)=\operatorname{card}(\gamma)$.

(2) If $\operatorname{card}(\gamma)$ is singular, then $\operatorname{card}(\Psi)<\operatorname{card}(\gamma)$. (This holds for the following reason. The cofinality operator $c f$ satisfies $c f(c f(\alpha))=c f(\alpha)$ for each ordinal $\alpha$. Therefore, if $c f(\gamma)=\operatorname{card}(\gamma)$, we obtain a contradiction as follows: $\operatorname{card}(\gamma)=c f(\gamma)=c f(c f(\gamma))=$ 
$c f(\operatorname{card}(\gamma))<\operatorname{card}(\gamma)$, where the last inequality is due to $\operatorname{card}(\gamma)$ being singular. Thus, $c f(\gamma)<\operatorname{card}(\gamma)$, whence $\operatorname{card}(\Psi)=c f(\gamma)<\operatorname{card}(\gamma)$, as required.)

By the inductive hypothesis, for every ordinal $\delta \in \Psi$, there is a suitable model $\mathcal{M}_{\delta}$, where $\mathcal{L}_{\mathrm{E}}\left(q_{0}\right)=\delta$. We build an isomorphic copy $\mathcal{M}_{\delta}^{\prime}=\left(\mathbb{A g t}, \mathrm{St}_{\delta}, \Pi, \mathrm{Act}_{\delta}, d_{\delta}, o_{\delta}, v_{\delta}\right)$ of every model $\mathcal{M}_{\delta}$ so that the sets $\mathrm{St}_{\delta}$ are disjoint and such that the state $q_{0}$ is replaced with a new state $q_{\delta}$ in every model $\mathcal{M}_{\delta}^{\prime}$. Now, let $\mathcal{M}_{\gamma}=(\mathbb{A g t}$, St, $\Pi$, Act, $d, o, v)$, where:

$$
\begin{aligned}
& -\mathrm{St}=\bigcup_{\delta \in \Psi} \mathrm{St}_{\delta} \cup\left\{q_{0}\right\}, \\
& -d=\bigcup_{\delta \in \Psi} d_{\delta} \cup\left\{\left(\left(q_{0}, a\right),\{\delta \mid \delta \in \Psi\}\right)\right\}, \\
& -o=\bigcup_{\delta \in \Psi} o_{\delta} \cup \bigcup_{\delta \in \Psi}\left\{\left(\left(q_{0}, \delta\right), q_{\delta}\right)\right\}, \\
& -v:=\left\{\left(p, \bigcup_{\delta \in \Psi} v_{\delta}(p)\right)\right\}, \text { that is, } v \text { is the function that maps } p \text { to the union of the sets } v_{\delta}(p) .
\end{aligned}
$$

Since $\sup (\Psi)=\gamma$, it is easy to see that $\mathcal{L}_{\mathrm{E}}\left(q_{0}\right)=\gamma$. Clearly, $\mathcal{M}_{\gamma}$ has the required properties, whence the transfinite induction for constructing the models $\mathcal{M}_{\gamma}$ has now been completed.

Before completing our proof, we define a further class of models $\mathcal{N}_{\mu}$ for each infinite cardinal $\mu$ such that $\mathcal{N}_{\mu}$ has a node $q^{\prime}$ with $\operatorname{BD}\left(q^{\prime}\right)=\mu$. These models $\mathcal{N}_{\mu}$ are defined simply for the sake of simplifying our subsequent arguments. Intuitively, the models $\mathcal{N}_{\mu}$ will be used in order to directly force the existence of sufficiently large branchings. Formally, we define $\mathcal{N}_{\mu}=\left(\mathbb{A g t}, \mathrm{St}_{\mu}, \Pi\right.$, Act $\left._{\mu}, d_{\mu}, o_{\mu}, v_{\mu}\right)$ as follows. We let $\mathbb{A g t}=\{a\}$ and $\mathrm{St}_{\mu}:=\left\{q^{\prime}\right\} \cup\left\{q_{\delta} \mid \delta<\right.$ $\mu, \delta$ is an ordinal $\}$. We also define $\Pi=\{p\}$ and let Act A $_{\mu}$ be the set of ordinals smaller than $\mu$. We define the set of actions available to agent $a$ at $q^{\prime}$ to be the set Act ${ }_{\mu}$, while the set of actions available elsewhere is defined arbitrarily. The outcome of $a$ choosing the action $\delta$ at $q^{\prime}$ will lead to $q_{\delta}$. At other states, the outcomes of actions are defined arbitrarily. Finally, we define $v_{\mu}(p)=\emptyset$.

To complete our proof, we argue as follows. Let $\kappa$ be an infinite cardinal. Assume first that $\kappa$ is regular. Let $\mathcal{M}$ be the disjoint union of the models $\mathcal{M}_{\gamma}$ and $\mathcal{N}_{\gamma}$ for all $\gamma<\kappa$ constructed above. Now, $\mathcal{M}$ is less than $\kappa$-branching, and since $\kappa$ is regular, we have that $\operatorname{RBB}(\mathcal{M})=\kappa$. Clearly, $\mathcal{M}$ realizes exactly all ordinals $\gamma<\operatorname{RBB}(\mathcal{M})=\kappa$, as required. (Recall that $\operatorname{RBB}(\mathcal{M})$ is a globally stable limit bound and thus no labels greater than or equal to $\operatorname{RBB}(\mathcal{M})$ can be realized in $\mathcal{M}$.) Assume, then, that $\kappa$ is singular. Now, let $\mathcal{M}$ be the disjoint union of the models $\mathcal{M}_{\gamma}$ for all $\gamma<\kappa^{+}$and the models $\mathcal{N}_{\gamma}$ for all $\gamma<\kappa$. Again, $\mathcal{M}$ is less than $\kappa$-branching. Since $\kappa$ is not regular, we have that $\operatorname{RBB}(\mathcal{M})=\kappa^{+}$. Thus, $\mathcal{M}$ realizes exactly all ordinals $\gamma<\operatorname{RBB}(\mathcal{M})$, as required.

Example 4.24. By the proof of Proposition 4.23, we can construct a model $\mathcal{M}$ whose branchings are strictly below the singular cardinal $\boldsymbol{\aleph}_{\omega}$; nevertheless, all of the winning time labels $\gamma<\boldsymbol{\aleph}_{\omega}^{+}=$ $\operatorname{RBB}(\mathcal{M})$ are realized in $\mathcal{M}$. Thus, if we know only that the branchings of an arbitrary model $\mathcal{N}$ are bounded by the strict least upper bound $\boldsymbol{\aleph}_{\omega}$, but know nothing else about the model, we cannot give any better estimate than $\operatorname{RBB}(\mathcal{N})=\boldsymbol{\aleph}_{\omega}^{+}$for a stable time limit bound for $\mathcal{N}$.

\subsection{Unbounded versus Bounded Embedded Games}

As mentioned earlier, $\infty$-canonical strategies $\tau_{\mathrm{P}}^{\infty}$ (recall Definition 4.8) are important in evaluation games with sufficiently large time limit bounds $\Gamma$. We will see that if the time limit bound $\Gamma$ is stable and there exists a winning strategy for each $\gamma<\Gamma$, then $\tau_{\mathrm{P}}^{\infty}$ will be a winning strategy for each $\gamma<\Gamma$. The intuition behind $\infty$-canonical strategies is that the player does not know the current time limit $\gamma$ (since the strategy depends on states only), but the player is playing "defensively" by always assuming $\gamma$ to have the highest possible value (i.e., $\Gamma-1$ ).

The following lemma shows that, when $\Gamma$ satisfies certain conditions, then if $\mathbf{P}$ uses an $\boldsymbol{\infty}-$ canonical strategy $\tau_{\mathrm{P}}^{\infty}$ and begins from a state with the label win, $\mathbf{P}$ will always stay in states with the label win. 
Lemma 4.25. Let $\mathrm{G}=\mathrm{g}\left(\mathrm{V}, \mathrm{C}, A, q_{0}, \psi_{\mathrm{C}}, \psi_{\overline{\mathrm{C}}}\right)$ be an embedded game, $\mathrm{P} \in\{\mathrm{A}, \mathrm{E}\}$, and $\mathbf{P} \neq \mathbf{C}$. Assume that the time limit bound $\Gamma$ is a successor ordinal and $\Gamma-1$ is stable for $\mathrm{G}$. Now, for every $q \in \mathrm{St}$, if $\mathcal{L}_{\mathbf{P}}(q)=$ win and $Q \subseteq$ St is forced by $\tau_{\mathrm{P}}^{\infty}$, then $\mathcal{L}_{\mathbf{P}}\left(q^{\prime}\right)=$ win for every $q^{\prime} \in Q$.

Proof. Suppose that $\mathcal{L}_{\mathrm{P}}(q)=$ win and $Q \subseteq$ St is forced by $\tau_{\mathrm{P}}^{\infty}(q)$. Let $\sigma_{\mathrm{P}}$ be a strategy for which $l\left(q, \sigma_{\mathrm{P}}\right)=$ win and $\tau_{\mathrm{P}}^{\infty}(q)=\sigma_{\mathrm{P}}(\Gamma-1, q)$ (such a strategy exists by Definition 4.8). For the sake of contradiction, suppose that there is some $q^{\prime} \in Q$ for which $\mathcal{L}_{\mathrm{P}}\left(q^{\prime}\right) \neq$ win and, thus, $\mathcal{L}_{\mathrm{P}}\left(q^{\prime}\right)=\gamma$ for some $\gamma<\Gamma$. Since $\Gamma-1$ is stable for $G$, we must also have that $\gamma<\Gamma-1$. Now, there is a play of $\mathrm{G}[q, \Gamma-1]$ in which $\mathbf{P}$ is using $\sigma_{\mathrm{P}}$ and the configuration $\left(\gamma, q^{\prime}\right)$ follows $(\Gamma-1, q)$. But since $\mathcal{L}_{\mathbf{P}}\left(q^{\prime}\right)=$ $\gamma$, the strategy $\sigma_{\mathrm{P}}$ cannot be a winning strategy in $\mathrm{G}\left[q^{\prime}, \gamma\right]$. Hence, $\sigma_{\mathrm{P}}$ is not a winning strategy in $\mathrm{G}[q, \Gamma-1]$, which is a contradiction since $l\left(q, \sigma_{\mathrm{P}}\right)=$ win.

The following proposition shows that when the time limit bound $\Gamma$ is stable, then bounded embedded games become essentially equivalent to unbounded embedded games. For the proof, we use concepts that we have defined before. The key here is to use a canonically timed strategy when $\mathbf{P}=\mathbf{C}$ and an $\infty$-canonical strategy when $\mathbf{P} \neq \mathbf{C}$.

Proposition 4.26. Let $\mathrm{G}=\mathrm{g}\left(\mathrm{V}, \mathrm{C}, A, q_{0}, \psi_{\mathrm{C}}, \psi_{\overline{\mathrm{C}}}\right.$, ) be an embedded game and let $\mathbf{P} \in\{\mathrm{A}, \mathrm{E}\}$. Suppose that $\Gamma$ is stable for $\mathrm{G}$. Then, the following equivalences hold-under the exceptional assumption that the winning condition of the exit positions of the unbounded embedded game G (recall Definition 4.1) is defined by using the semantics $\left.\right|_{\Gamma} ^{g}$ instead of $\left.\right|_{u_{u}^{g}} ^{g}$.
-If $\mathbf{P}=\mathrm{C}$, then there is a winning strategy $\sigma_{\mathrm{P}}$ in $\mathrm{G}$ iff there is $\gamma_{0}<\Gamma$ and a timed winning strategy $\left(\sigma_{\mathrm{P}}^{\prime}, t\right)$ in $\mathrm{G}\left[\gamma_{0}\right]$.
-If $\mathbf{P} \neq \mathbf{C}$, then there is a winning strategy $\sigma_{\mathrm{P}}$ in $\mathrm{G}$ iff there is $\sigma_{\mathrm{P}}^{\prime}$, which is a winning strategy in $\mathrm{G}\left[\gamma_{0}\right]$ for every $\gamma_{0}<\Gamma$.

Proof. Suppose first that $\mathbf{P}=\mathbf{C}$. If $\left(\sigma_{\mathrm{P}}^{\prime}, t\right)$ is a timed winning strategy in $\mathrm{G}\left[\gamma_{0}\right]$ for some time limit $\gamma_{0}<\Gamma$, then, by Proposition $4.10,\left(\tau_{\mathrm{P}}, t_{c a n}\right)$ is a timed winning strategy in $\mathrm{G}\left[\gamma_{0}\right]$. Now, the strategy $\tau_{\mathrm{P}}$ will also be a winning strategy in $\mathrm{G}$ (note that infinite plays are impossible with $\tau_{\mathrm{P}}$ and that $\tau_{\mathrm{P}}$ depends on states only).

For the other direction, suppose that there is a winning strategy $\sigma_{\mathbf{P}}$ for $\mathbf{P}$ in $\mathbf{G}$. We assume, for the sake of contradiction, that $\mathbf{P}$ does not have a timed winning strategy in $G\left[\gamma_{0}\right]$ for any $\gamma_{0}<\Gamma$. Hence, by Proposition 4.4, we have that $\mathcal{L}_{\mathbf{P}}\left(q_{0}\right)=$ lose. As a consequence of Proposition 4.13, we must now have that $\mathcal{L}_{\overline{\mathbf{P}}}\left(q_{0}\right)=$ win. Let $\Gamma^{\prime}:=\Gamma+1$. We construct an $\infty$-canonical strategy $\tau_{\overline{\mathbf{P}}}^{\infty}$ for $\overline{\mathbf{P}}$ by using the strategies that correspond to embedded games with the time limit bound $\Gamma^{\prime}$. Since $\Gamma$ is stable, the winning time labels of the states will not change when $\Gamma$ is increased and, in particular, the state $q$ will still have the value win for $\overline{\mathbf{P}}$. Now, the assumptions of Lemma 4.25 hold; thus, we can use it to deduce that all (finite) plays of $\mathrm{G}$ with $\tau_{\overline{\mathrm{P}}}^{\infty}$ will end at a state that has the label win for $\overline{\mathbf{P}}$. But, in order to lose $\mathrm{G}$, the player $\overline{\mathbf{P}}$ should end up at a state with the label 0 . Hence, $\tau_{\overline{\mathbf{P}}}^{\infty}$ must be a winning strategy for $\overline{\mathbf{P}}$ in $\mathrm{G}$ (note here that $\tau_{\mathrm{P}}^{\infty}$ depends on states only and thus may be used in $\mathrm{G})$. This is a contradiction since we assumed that $\mathrm{P}$ has a winning strategy in $\mathrm{G}$.

Suppose, then, that $\mathbf{P} \neq \mathbf{C}$. If there is a winning strategy $\sigma_{\mathbf{P}}$ for $\mathbf{P}$ in $\mathbf{G}$, then we can define $\sigma_{\mathbf{P}}^{\prime}(\gamma, q)=\sigma_{\mathbf{P}}(q)$ for every $\gamma<\Gamma$, whence $\sigma_{\mathbf{P}}^{\prime}$ will be a winning strategy in $\mathrm{G}\left[\gamma_{0}\right]$ for every time limit $\gamma_{0}<\Gamma$.

\footnotetext{
${ }^{4}$ However, we will see (by Theorem 4.27) that when $\Gamma$ is globally stable for $\mathcal{M}$, then these two notions of truth become equivalent and, thus, this exceptional assumption here becomes irrelevant. (Also, note that the exceptional assumption can only be considered relevant in situations in which another "nested" embedded game can be played after playing G.)
} 
For the other direction, suppose that there is a strategy $\sigma_{\mathrm{P}}^{\prime}$ that is a winning strategy in $\mathrm{G}\left[\gamma_{0}\right]$ for every time limit $\gamma_{0}<\Gamma$. Then, by Proposition 4.4 , we have that $\mathcal{L}_{\mathbf{P}}\left(q_{0}\right)=$ win. As above, we can now increase $\Gamma$ to $\Gamma+1$ in order to construct an $\infty$-canonical strategy $\tau_{\mathbf{P}}^{\infty}$ for $\mathbf{P}$ in $\mathbf{G}$. With the same reasoning as above, $\tau_{\mathrm{P}}^{\infty}$ will be a winning strategy for $\mathbf{P}$ in $\mathrm{G}$.

By using Proposition 4.26, we can now prove the equivalence of unbounded and $\Gamma$-bounded game-theoretic semantics for a stable time limit bound $\Gamma$.

Theorem 4.27. Let $\mathcal{M}$ be a CGM, $q \in \mathrm{St}$, and $\varphi$ an ATL-formula. Suppose that $\Gamma>0$ is a globally stable time limit bound for $\mathcal{M}$. Then, we have that

$$
\mathcal{M}, q \models_{u}^{g} \varphi \text { iff } \mathcal{M}, q \models_{\Gamma}^{g} \varphi .
$$

Proof. We prove by induction on $\varphi$ that Eloise has a winning strategy in $\mathcal{G}(\mathcal{M}, q, \varphi)$ if and only if she has a winning strategy in $\mathcal{G}(\mathcal{M}, q, \varphi, \Gamma)$. Since the rules for unbounded and bounded evaluation games differ only for positions that lead to embedded games, we need to consider only the cases in which $\varphi=\psi \cup \theta$ or $\varphi=\psi \mathrm{R} \theta$.

Let $\mathrm{G}$ be the unbounded embedded game that arises from $\varphi$. Since $\Gamma$ is globally stable for the model $\mathcal{M}$, it is stable for $\mathrm{G}$. By the inductive hypothesis, the equivalence of the two semantics holds for the formulae $\psi$ and $\theta$. We may thus assume that the winning condition of the exit positions of $\mathrm{G}$ has been defined by using $\Gamma$-bounded GTS instead of the unbounded GTS (recall the corresponding assumption in Proposition 4.26). Since $\Gamma$-bounded evaluation games are determined (Proposition 4.15), it now follows that having a winning strategy in $\mathrm{G}-$ or in $\mathrm{G}\left[\gamma_{0}\right]$ for some $\gamma_{0}<\Gamma$-guarantees having a winning strategy in the corresponding evaluation game that is continued (recall Definition 4.1). The inductive step for $\varphi$ can thus be proven easily by using Proposition 4.26.

If Eloise is the controller in G, then, by Proposition 4.26, she has a winning strategy in $\mathrm{G}$ if and only if there is some time limit $\gamma<\Gamma$ such that she has a (timed) winning strategy in G $[\gamma]$. If Eloise is not the controller in $\mathrm{G}$, then, by Proposition 4.26, she has a winning strategy in $\mathrm{G}$ if and only if she has a winning strategy in $G[\gamma]$ for every $\gamma<\Gamma$ (chosen by the Abelard). The claim for $\varphi$ thus follows.

Since $\Gamma$-bounded evaluation games are positionally determined for any time limit bound $\Gamma>0$ (by Proposition 4.15), in particular, they are determined for such $\Gamma$ that are globally stable for a given model $\mathcal{M}$. Hence, Theorem 4.27 implies that unbounded evaluation games (and unbounded embedded games) are also positionally determined. By these results, we see that even if we had defined memory-based strategies for bounded or unbounded evaluation games, the resulting semantics would have remained equivalent to the current one.

As a direct corollary of Theorem 4.27, we obtain the equivalence of unbounded and bounded game-theoretic semantics.

Corollary 4.28. Let $\mathcal{M}$ be a CGM, $q \in \mathrm{St}$, and $\varphi$ an ATL-formula. Then,

$$
\mathcal{M},\left.q\right|^{g}{ }_{u}^{g} \varphi \text { iff } \mathcal{M},\left.q\right|_{b} ^{g} \varphi .
$$

Proof. Assume that $\operatorname{card}(\mathcal{M})=\kappa$. By Corollary 4.22(1), the ordinal $2^{\kappa}$ is globally stable for $\mathcal{M}$; thus, the claim follows from Theorem 4.27.

This proof relies on the (global) stability of the time limit bound $\Gamma=2^{\operatorname{card}(\mathcal{M})}$. We know by Proposition 4.21 that the time limit bound $\Gamma=\operatorname{RBB}(\mathcal{M})$ would also suffice for this proof. However, by Proposition 4.23 , for any time limit bound $\Gamma<\operatorname{RBB}(\mathcal{M})$, there are cases in which $\Gamma$-bounded semantics is not equivalent with the unbounded GTS. Therefore, we cannot use any fixed value of $\Gamma$ in order to obtain this equivalence. 
Even though the finitely bounded semantics is not equivalent to the unbounded semantics (see Example 3.11), the three semantics become equivalent on a natural class of models.

Theorem 4.29. Let $\mathcal{M}$ be an image-finite CGM, $q \in \mathrm{St}$, and $\varphi$ an ATL-formula. Then,

$$
\mathcal{M}, q \mid{ }_{f}^{g} \varphi \text { iff } \mathcal{M},\left.q\right|_{u} ^{g} \varphi \text { iff } \mathcal{M},\left.q\right|_{b} ^{g} \varphi .
$$

Proof. Since $\mathcal{M}$ is image finite, by Corollary 4.22(2), the ordinal $\omega$ is a globally stable time limit bound for $\mathcal{M}$; thus, the claim follows from Theorem 4.27 and Corollary 4.28.

Note that in image-finite models, all ordinal-valued winning time labels are finite. Thus, the controller would gain nothing from being able to use infinite ordinals in embedded games.

\section{GAME-THEORETIC VERSUS COMPOSITIONAL SEMANTICS}

\subsection{Equivalence between the Unbounded GTS and the Compositional Semantics}

We show that the unbounded game-theoretic semantics for ATL is equivalent to the standard compositional semantics of ATL.

Theorem 5.1. Let $\mathcal{M}$ be a CGM, $q_{\text {in }} \in \mathrm{St}$, and $\varphi$ an ATL-formula. Then,

$$
\mathcal{M}, q_{\text {in }}=\varphi \text { iff } \mathcal{M}, q_{\text {in }} \models^{g} \stackrel{\text { u }}{ } \text {. }
$$

Proof. (Sketch) The equivalence can be proven by induction on $\varphi$. The cases $\varphi=p$ and $\varphi=$ $\psi \vee \theta$ are easy. For the case $\varphi=\neg \psi$, we need to use the determinacy of the evaluation games from Proposition 4.15. In the cases for strategic operators $\langle\langle A\rangle \mathrm{X} \psi,\langle\langle A\rangle \psi \cup \theta$, and $\langle\langle A\rangle \psi \mathrm{R} \theta$, we construct a strategy $\sigma_{\mathrm{P}}$ for the embedded (or one step) game using the collective strategy $S_{A}$ and vice versa. For a detailed proof, see the appendix.

By Theorem 4.28, we immediately obtain the following corollary.

Corollary 5.2. Let $\mathcal{M}$ be a CGM, $q_{\text {in }} \in \mathrm{St}$, and $\varphi$ an ATL-formula. Then,

$$
\mathcal{M}, q_{\text {in }}=\varphi \text { iff } \mathcal{M},\left.q_{\text {in }}\right|^{g}{ }_{b}^{g} \varphi \text {. }
$$

However, as we have discussed in Section 4.5, $\Gamma$-bounded GTS is not equivalent with the unbounded GTS for any fixed value of the time limit bound $\Gamma$. Therefore, the different values of $\Gamma$ lead to different semantic systems that are all nonequivalent to the standard compositional semantics.

\subsection{Finitely Bounded Compositional Semantics}

As shown earlier (Example 3.11), the finitely bounded game-theoretic semantics is not equivalent to the standard compositional semantics of ATL. However, it can still be shown to be equivalent to a natural semantics, to be defined next, which we call finitely bounded compositional semantics.

Definition 5.3. Let $\mathcal{M}=(\mathbb{A g t}, \mathrm{St}, \Pi, \mathrm{Act}, d, o, v)$ be a CGM, $q \in \mathrm{St}$, and $\varphi$ an ATL-formula. The truth of $\varphi$ in $\mathcal{M}$ at $q$ according to finitely bounded compositional semantics, denoted by $\mathcal{M},\left.q\right|_{f} \varphi$, is defined recursively as follows:

- The truth conditions for $p \in \Pi, \neg \psi, \psi \vee \theta$, and $\langle\langle A\rangle \mathrm{X} \psi$ are as in the standard compositional semantics of ATL (see Definition 2.3).

$-\mathcal{M},\left.q\right|_{f}\left\langle\langle A\rangle \psi \cup \theta\right.$ iff there exists $n<\omega$ and a collective strategy $S_{A}$ such that for each $\Lambda \in$ paths $\left(q, S_{A}\right)$, there is some $i \leq n$ such that $\mathcal{M}, \Lambda[i]||_{f} \theta$ and $\mathcal{M}, \Lambda[j]||_{f} \psi$ for every $j<i$.

$-\mathcal{M},\left.q\right|_{f}\langle\langle A\rangle\rangle \psi \mathrm{R} \theta$ iff for every $n<\omega$, there exists a collective strategy $S_{A, n}$ such that for each $\Lambda \in$ paths $\left(q, S_{A, n}\right)$ and $i \leq n$, either $\mathcal{M},\left.\Lambda[i]\right|_{f} \theta$ or there is $j<i$ such that $\mathcal{M}, \Lambda[j] \models_{f}$ $\psi$. 
For $F$ and $G$, we obtain the following derived truth conditions:

$-\mathcal{M}, q \mid=_{b}\left\langle\langle A\rangle \mathrm{F} \psi\right.$ iff there exists $n<\omega$ and a collective strategy $S_{A}$ such that for each $\Lambda \in$ paths $\left(q, S_{A}\right)$ there is $i \leq n$ such that $\mathcal{M}, \Lambda[i] \models_{f} \psi$.

$-\mathcal{M},\left.q\right|_{f}\left\langle\langle A\rangle \mathrm{G} \psi\right.$ iff for every $n \geq 0$ there exists a collective strategy $S_{A, n}$ such that for each $\Lambda \in$ paths $\left(q, S_{A, n}\right)$ and $i \leq n$, we have that $\mathcal{M},\left.\Lambda[i]\right|_{f} \psi$.

There exists an interesting conceptual link between for-loops and while-loops on one hand and the finitely bounded and unbounded semantics of ATL on the other hand. This link is perhaps easiest to understand via the game-theoretic approaches to ATL semantics, but it ultimately makes no difference whether compositional or game-theoretic semantics is considered. The principal difference between the two types of loops is that for-loops require an input parameter, let us call it an iterator, which determines how many times the loop is executed. The iterator can be an input from elsewhere in the program or even a fixed constant (although, in the latter case, it is not clear whether the construct in question should be regarded as a genuine loop). Similarly, in the finitely bounded semantics, the execution of strategic formulae requires a finite input integer that limits the number of steps to be taken in the model and is clearly analogous to the iterator. While-loops and the unbounded semantics are similarly analogous to each other, both being essentially iterative processes but without an iterator bounding the number of executions.

It turns out that the finitely bounded compositional semantics simplifies, in a sense, the meaning of the temporal operators $U$ and $R$ and, in particular, of $F$ and $G$, by reducing them to simple infinitary disjunctions and conjunctions of iteration patterns using $X$ (see below). The iteration patterns correspond to finite approximations of the respective fixed-point characterizations (see Goranko and van Drimmelen (2006)). Recall that the difference between finitely bounded and the standard compositional semantics is essentially described by Example 3.11.

To characterize the finitely bounded semantics, we now define series of "iteration operators." For the sake of better illustration, we will treat only the cases of $G$ and $U$ here.

In what follows, $A$ denotes an arbitrary coalition of agents. First, we define the following operators on ATL formulae:

$$
\begin{aligned}
& -\mathrm{G}_{A ; \theta}(\varphi):=\theta \wedge\langle\langle A\rangle\rangle \mathrm{X} \varphi \\
& -\mathrm{U}_{A ; \psi, \theta}(\varphi):=\theta \vee(\psi \wedge\langle\langle A\rangle \mathrm{X} \varphi) .
\end{aligned}
$$

Now, we define the following recursively over $n \in \mathbb{N}$ :

$$
\begin{aligned}
& -\mathrm{G}_{A}^{0}(\theta):=\theta ; \mathrm{G}_{A}^{n+1}(\theta):=\mathrm{G}_{A ; \theta}\left(\mathrm{G}_{A}^{n}(\theta)\right) \\
& -\mathbf{U}_{A}^{0}(\psi, \theta):=\theta ; \mathbf{U}_{A}^{n+1}(\psi, \theta):=\mathbf{U}_{A ; \psi, \theta}\left(\mathbf{U}_{A}^{n}(\psi, \theta)\right) .
\end{aligned}
$$

Lemma 5.4. For every CGM $\mathcal{M}$ and $q \in \mathcal{M}$, the following hold:

(1) $\mathcal{M},\left.q\right|_{f}\left\langle\langle A\rangle \mathrm{G} \theta\right.$ iff $\mathcal{M},\left.q\right|_{f} \mathrm{G}_{A}^{n}(\theta)$ for every $n \in \mathbb{N}$. Furthermore, if $\theta$ only contains occurrences of strategic operators of the type $\langle\langle B\rangle \mathrm{X}$, then $\mathcal{M}, q \mid=_{f}\langle\langle A\rangle \mathrm{G} \theta$ iff $\mathcal{M}, q| \mathrm{G}_{A}^{n}(\theta)$ for every $n \in \mathbb{N}$.

(2) $\mathcal{M}, q \models_{f}\left\langle\langle A\rangle \psi \cup \theta\right.$ iff $\mathcal{M}, q \models_{f} \mathbf{U}_{A}^{n}(\psi, \theta)$ for some $n \in \mathbb{N}$. Furthermore, if $\psi$ and $\theta$ only contain occurrences of strategic operators of the type $\langle\langle B\rangle\rangle$, then $\mathcal{M},\left.q\right|_{f}\langle\langle A\rangle \psi \cup \theta$ iff $\mathcal{M}, q|=\mathrm{U}_{A}^{n}(\psi, \theta)$ for some $n \in \mathbb{N}$.

These equivalences suggest how to modify the axioms characterizing these operators provided in Goranko and van Drimmelen (2006), that is, to replace the biconditionals

$$
\begin{aligned}
& \mathbf{F P}_{\mathbf{G}}:(\varphi \wedge\langle\langle A\rangle \mathrm{X}\langle\langle A\rangle \mathrm{G} \varphi) \leftrightarrow\langle\langle A\rangle\rangle \mathrm{G} \varphi \text { and } \\
& \mathbf{F P}_{\mathbf{U}}:\langle\langle A\rangle\rangle \varphi \mathrm{U} \psi \leftrightarrow(\psi \vee(\varphi \wedge\langle\langle A\rangle\rangle \mathrm{X}\langle\langle A\rangle\rangle \varphi \mathrm{U} \psi))
\end{aligned}
$$


by the respective implications

$\operatorname{PreFP}_{\mathbf{G}}:(\varphi \wedge\langle\langle A\rangle\rangle \mathrm{X}\langle\langle A\rangle\rangle \mathrm{G} \varphi) \rightarrow\langle\langle A\rangle\rangle \mathrm{G} \varphi$ and

PostFP $_{\mathbf{U}}:\langle\langle A\rangle\rangle \varphi \mathrm{U} \psi \rightarrow(\psi \vee(\varphi \wedge\langle\langle A\rangle\rangle \mathrm{X}\langle\langle A\rangle\rangle \varphi \mathrm{U} \psi))$.

Lemma 5.4 has also partly been discussed in the follow-up work (Goranko et al. 2018), in which we present a sound and complete infinitary axiomatization for the finitely bounded compositional semantics of ATL by adding respective infinitary axiom schemes and inference rules.

\subsection{Equivalence between the Finitely Bounded Compositional and Game-Theoretic Semantics}

To prove equivalence between the finitely bounded compositional semantics and the finitely bounded GTS, we need to show that it is sufficient to consider strategies in the embedded games that depend on states only. This property will be needed because collective strategies for coalitions $\left(S_{A}\right)$ in the compositional semantics of ATL formulae are positional (according to Definition 2.3).

LEMMA 5.5. If Eloise has a winning strategy $\Sigma_{\mathrm{E}}$ in a finitely bounded evaluation game $\mathcal{G}\left(\mathcal{M}, q_{i n}, \varphi, \omega\right)$, then she has a winning strategy $\Sigma_{\mathbf{E}}^{\prime}$ that uses, in every bounded embedded game, a strategy $\sigma_{\mathrm{E}}$ that depends only on states.

Proof. In summary, the idea is that if $\mathrm{E}=\mathrm{C}$ in some embedded game, Eloise may play with the canonical strategy $\tau_{\mathrm{E}}$ that depends only on states. If $\mathbf{E} \neq \mathbf{C}$ and Abelard chooses $n<\omega$ as the time limit, Eloise may play with the $n$-canonical strategy $\tau_{\mathrm{E}}^{n}$ that only depends on states. A more detailed explanation follows.

Suppose that $\Sigma_{\mathrm{E}}$ is a winning strategy in a finitely bounded evaluation game $\mathcal{G}\left(\mathcal{M}, q_{i n}, \varphi, \omega\right)$. Let $\mathbf{G}=\mathbf{g}\left(\mathbf{V}, \mathbf{C}, A, q_{0}, \psi_{\mathbf{C}}, \psi_{\overline{\mathrm{C}}}\right)$ be an embedded game that is related to some position Pos in the evaluation game $\mathcal{G}\left(\mathcal{M}, q_{i n}, \varphi, \omega\right)$. Suppose first that $\mathrm{E}=\mathrm{C}$. Then, $\Sigma_{\mathrm{E}}(\operatorname{Pos})=\left(n, \sigma_{\mathrm{E}}\right)$ for some $n<\omega$ (the timer $t$ is not used in the finitely bounded case; thus, it can be omitted here). Since $\Sigma_{\mathrm{E}}$ is a winning strategy in the evaluation game, $\sigma_{\mathrm{E}}$ must be a winning strategy in the embedded game $\mathrm{G}[n]$. By Proposition 4.10, the canonical strategy $\tau_{\mathrm{E}}$ is a winning strategy in $\mathrm{G}[n]$ (the canonical timer is not needed here). Suppose now that $\mathrm{E} \neq \mathrm{C}$. Then, $\Sigma_{\mathrm{E}}$ (Pos) maps every $n<\omega$ to some strategy $\sigma_{\mathrm{E}, n}$. Since $\Sigma_{\mathrm{E}}$ is a winning strategy in the evaluation game, $\sigma_{\mathrm{E}, n}$ must be a winning strategy in the corresponding bounded embedded game $\mathrm{G}[n]$. By Lemma 4.11 , for every $n<\omega$, the $n$-canonical strategy $\tau_{\mathrm{E}}^{n}$ is a winning strategy in $\mathrm{G}[n]$.

Thus, it is easy to see that we can construct a strategy $\Sigma_{\mathrm{E}}^{\prime}$ for Eloise in such a way that it only uses canonical strategies when $\mathrm{E}=\mathrm{C}$ and maps all $n<\omega$ to $n$-canonical strategies when $\mathrm{E} \neq \mathrm{C}$. Since these strategies depend on states only, the claim now follows for $\Sigma_{\mathbf{E}}^{\prime}$.

Using the previous lemma, we can now prove the equivalence between the finitely bounded compositional and game-theoretic semantics using a similar induction as the one in the proof of Theorem 5.1. See the proof in the appendix.

Theorem 5.6. Let $\mathcal{M}$ be a CGM, $q_{i n} \in \mathrm{St}$, and $\varphi$ an ATL-formula. Then,

$$
\mathcal{M},\left.q_{\text {in }}\right|_{f} \varphi \text { iff } \mathcal{M},\left.q_{\text {in }}\right|_{f} ^{g} \varphi .
$$

As we have seen in Example 3.11, the fixed-point characterization of the temporal operator $F$ fails with finitely bounded semantics (both compositional and game-theoretic). However, since finitely bounded GTS is equivalent with the standard semantics in image-finite models (in particular, finite models), the formula $\neg\langle\langle A\rangle\rangle \mathrm{F} p \wedge(p \vee\langle\langle A\rangle\rangle \mathrm{X}\langle\langle A\rangle \mathrm{F} p)$ is satisfied only in infinite models. Therefore, we obtain the following result:

Proposition 5.7. ATL with finitely bounded semantics lacks the finite model property. 
It is well known that ATL with the standard semantics has the finite model property. By this fact, and the equivalence of the finitely bounded and the standard semantics in finite models, we obtain the following interesting consequence.

COROLLARY 5.8. All validities of ATL with finitely bounded semantics are also valid with the standard semantics.

\section{Concluding Remarks}

Game-theoretic approaches have proved to be a natural and fruitful idea in many areas of logic. The principal aim of this article has been to develop a conceptual and technical framework for systems of game-theoretic semantics for ATL. The game-theoretic perspective appears in the setting of ATL on two semantic levels: on the object level, in the standard semantics of the strategic operators, and on the meta-level, where game-theoretic logical semantics is applied to formulae. We have unified these two perspectives in the semantic evaluation games designed here for ATL and argued that the resulting versions of game-theoretic semantics are conceptually and technically natural from logical as well as game-theoretic perspectives. Our bounded GTS provides a new framework in which truth of ATL-formulae can be established in finite time-even on infinite models-despite the fact that ATL-formulae involve temporal operators that refer to infinite computations.

The ideas and results in the present work can be naturally extended to other richer languages. In particular, Goranko et al. (2017b) introduces GTS for the extended logic $\mathrm{ATL}^{+}$. Because of the more expressive language of $\mathrm{ATL}^{+}$, which generally requires memory-based strategies, the GTS for that logic is considerably more complex, while formally extending the GTS for ATL developed here. In other recent work, Hella et al. (2017) introduce a bounded semantics for the modal $\mu$ calculus, directly inspired by the approach in this article based on ordinals. This leads to games alternative to the usual parity games in which the durations of plays are always guaranteed to be finite. However, Hella et al. (2017) discuss the case for standard modalities rather than the strategic operators used here.

The finitely bounded compositional semantics for ATL and other expressive branching time logics, naturally emerging from the present work, is of particular interest for several reasons and deserves a separate study of its own. As a first step in that direction, in a recent work (Goranko et al. 2017a), we have studied in more detail the finitely bounded semantics for the computational tree logic CTL (which can be seen as a single-agent variant of ATL). In particular, we have presented there sound and complete tableaux systems for checking the validities of CTL with finitely bounded semantics, one of which is infinitary, while the other is finitary and provides an EXPTIME decision procedure for the satisfiability problem for that logic. Thus, we establish EXPTIME-completeness of the logic, the same complexity as that of standard CTL. In Goranko et al. (2018), we extend these results and provide an infinitary complete axiomatization for ATL with finitely bounded semantics.

We will now briefly address two important issues on how the present work links to other research. The first issue is-as already pointed out in the introduction-that some of our technical results could have alternatively been derived using some general results for coalgebraic modal logic. This is because concurrent game models can be viewed as coalgebras for a game functor defined in Cîrstea et al. (2011), and the fixed-point extension of the coalitional coalgebraic modal logic for this functor links to ATL in a natural way. Game-theoretic semantics has been developed for coalgebraic fixed-point logics in Venema (2006), Cîrstea et al. (2009), and Fontaine et al. (2010) and could be used to obtain some of our results concerning the unbounded game-theoretic semantics. However, as general and powerful as that approach is, it would not be very helpful for readers not familiar with coalgebras and coalgebraic modal logic; thus, the more direct and self-contained approach adopted here has its benefits. Moreover, our work on bounded and finitely bounded semantics is not directly related to existing work in coalgebraic modal logic, though even there some 
natural shortcuts based on background theory could have been used. For example, using König's Lemma, it is easy to prove that the finitely bounded and unbounded game-theoretic semantics are equivalent on image-finite models.

The second issue concerns links to alternating Turing machines and automata and some possible applications. In addition to defining variants of GTS for ATL, we have identified a number of related useful concepts, such as $n$-canonical and $\infty$-canonical strategies and winning time labels for embedded games. All of these could also be useful outside the context of ATL. In particular, we believe that the finitely bounded game-theoretic semantics can be used to connect different kinds of extensions of ATL to alternating Turing machines (and alternating automata) as well as different kinds of games. Recalling that the size of the model domain is a sufficient initial time limit for semantic games in finite models, it is easy to see that evaluation games directly translate in polynomial time to equivalent alternating reachability games, thereby providing a direct proof of the PTIME upper bound of the model checking problem of ATL. A similar approach works also in the context of various extensions of ATL, such as $\mathrm{ATL}^{+}$, as demonstrated in Goranko et al. (2017b), in which some known and some new results on the complexity of model checking $\mathrm{ATL}^{+}$and some of its fragments have been obtained by using these links.

\section{APPENDIX}

\section{A SOME TECHNICAL PROOFS}

\section{Proof of Lemma 4.3}

We define the strategy $\sigma_{\mathrm{P}}^{\prime}$ for any configuration $(\gamma, q)$, where $\gamma<\Gamma$ and $q \in \mathrm{St}$, as follows.

-If $\left(\sigma_{\mathrm{P}}, t\right)$ is not a timed winning strategy in $\mathrm{G}\left[q, \gamma^{\prime}\right]$ for any $\gamma^{\prime} \leq \gamma$, set $\sigma_{\mathrm{P}}^{\prime}(\gamma, q)=$ any.

-Else, set $\sigma_{\mathrm{P}}^{\prime}(\gamma, q)=\sigma_{\mathrm{P}}\left(\gamma^{\prime}, q\right)$, where $\gamma^{\prime} \leq \gamma$ is the smallest ordinal such that $\left(\sigma_{\mathrm{P}}, t\right)$ is a timed winning strategy in $\mathrm{G}\left[q, \gamma^{\prime}\right]$.

We define the timer $t^{\prime}$ for any pair $(\gamma, q)$, where $\gamma<\Gamma$ is a limit ordinal and $q \in \mathrm{St}$, in the following way.

-If $\left(\sigma_{\mathrm{P}}, t\right)$ is not a timed winning strategy in $\mathrm{G}\left[q, \gamma^{\prime}\right]$ for any $\gamma^{\prime}<\gamma$, set $t^{\prime}(\gamma, q)=$ any.

-Else, $t^{\prime}(\gamma, q)=\gamma^{\prime}$, where $\gamma^{\prime}$ is any ordinal such that $\gamma^{\prime}<\gamma$ and $\left(\sigma_{\mathrm{P}}, t\right)$ is a timed winning strategy in $\mathrm{G}\left[q, \gamma^{\prime}\right]$.

We prove by transfinite induction on $\gamma<\Gamma$ for every $q \in$ St that if $\left(\sigma_{\mathrm{P}}, t\right)$ is a timed winning strategy in $\mathrm{G}[q, \delta]$ for some $\delta \leq \gamma$, then $\left(\sigma_{\mathrm{P}}^{\prime}, t^{\prime}\right)$ is a timed winning strategy in $\mathrm{G}[q, \gamma]$. The claim then follows.

Let the inductive hypothesis be that the claim holds for every $\gamma^{\prime}<\gamma$ and suppose that $\left(\sigma_{\mathbf{P}}, t\right)$ is a timed winning strategy in $\mathrm{G}[q, \delta]$ for some $\delta \leq \gamma$. Let $\delta^{\prime}<\Gamma$ be the smallest ordinal such that $\left(\sigma_{\mathrm{P}}, t\right)$ is a timed winning strategy in $\mathrm{G}\left[q, \delta^{\prime}\right]$. Now, $\delta^{\prime} \leq \delta \leq \gamma$ and $\sigma_{\mathrm{P}}^{\prime}(\gamma, q)=\sigma_{\mathrm{P}}\left(\delta^{\prime}, q\right)$.

Suppose first that $\delta^{\prime}=0$, whence $\left(\sigma_{\mathrm{P}}, t\right)$ is a timed winning strategy in $\mathrm{G}[q, 0]$. Now, $\sigma_{\mathrm{P}}^{\prime}(\gamma, q)=$ $\sigma_{\mathrm{P}}(0, q)=\psi_{\mathrm{C}}$ and, thus, $\left(\sigma_{\mathrm{P}}^{\prime}, t^{\prime}\right)$ is a timed winning strategy in $\mathrm{G}[q, \gamma]$.

Suppose, then, that $\delta^{\prime}>0$, whence we must have that $\sigma_{\mathrm{P}}\left(\delta^{\prime}, q\right) \neq \psi_{\mathrm{C}}$. Let $q^{\prime} \in$ St be any possible successor state of $q$ when $\mathbf{P}$ follows $\sigma_{\mathrm{P}}\left(\delta^{\prime}, q\right)$. Now, $\left(\sigma_{\mathrm{P}}, t\right)$ must be a timed winning strategy in $\mathrm{G}\left[q^{\prime}, \delta^{\prime \prime}\right]$ for some ordinal $\delta^{\prime \prime}<\delta^{\prime}$ (if $\delta^{\prime}$ is a limit ordinal, then $\delta^{\prime \prime}=t\left(\delta^{\prime}, q^{\prime}\right)$, and if $\delta^{\prime}$ is a successor ordinal, then $\left.\delta^{\prime \prime}=\delta^{\prime}-1\right)$. Since $\delta^{\prime} \leq \gamma$, we have that $\delta^{\prime \prime}<\gamma$.

For the inductive step, suppose first that $\gamma$ is a successor ordinal. Since we have that $\delta^{\prime \prime}<\gamma$, we infer by the inductive hypothesis that $\left(\sigma_{\mathrm{P}}^{\prime}, t^{\prime}\right)$ is a timed winning strategy in $\mathrm{G}\left[q^{\prime}, \gamma-1\right]$. Hence, we see that $\left(\sigma_{\mathrm{P}}^{\prime}, t^{\prime}\right)$ must be a timed winning strategy in $\mathrm{G}[q, \gamma]$. Suppose, then, that $\gamma$ is a limit ordinal. Since $\left(\sigma_{\mathrm{P}}, t\right)$ is a timed winning strategy in $\mathrm{G}\left[q^{\prime}, \delta^{\prime \prime}\right]$, the value of $t^{\prime}\left(\gamma, q^{\prime}\right)$ is defined such 
that $t^{\prime}\left(\gamma, q^{\prime}\right)<\gamma$ and $\left(\sigma_{\mathrm{P}}, t\right)$ is a timed winning strategy in $\mathrm{G}\left[q^{\prime}, t^{\prime}\left(\gamma, q^{\prime}\right)\right]$. Thus, by the inductive hypothesis, $\left(\sigma_{\mathrm{P}}^{\prime}, t^{\prime}\right)$ is a timed winning strategy in $\mathrm{G}\left[q^{\prime}, t^{\prime}\left(\gamma, q^{\prime}\right)\right]$. Hence, we see that $\left(\sigma_{\mathrm{P}}^{\prime}, t^{\prime}\right)$ must be a timed winning strategy in $\mathrm{G}[q, \gamma]$.

\section{Proof of Proposition 4.4}

1. We first consider the case in which $\mathbf{P}=\mathbf{C}$.

(i) Suppose first that $\mathcal{L}_{\mathbf{P}}(q)=\gamma<\Gamma$. By Definition 4.2, there is some strategy $\sigma_{\mathrm{P}}$ whose strategy label $l\left(q, \sigma_{\mathrm{P}}\right)$ is $\gamma$. Thus, there is some timer $t$ such that the pair $\left(\sigma_{\mathrm{P}}, t\right)$ is a timed winning strategy for $\mathbf{P}$ in $\mathrm{G}[q, \gamma]$. By Lemma 4.3 , there is a pair $\left(\sigma_{\mathrm{P}}^{\prime}, t^{\prime}\right)$ that is a timed winning strategy in $\mathrm{G}\left[q, \gamma^{\prime}\right]$ for any $\gamma^{\prime}$ such that $\gamma \leq \gamma^{\prime}<\Gamma$. If there existed some timed winning strategy $\left(\sigma_{\mathbf{P}}^{\prime \prime}, t^{\prime \prime}\right)$ for $\mathbf{P}$ in $\mathrm{G}\left[q, \gamma^{\prime}\right]$ for some $\gamma^{\prime}<\gamma$, then we would have that $l\left(q, \sigma_{\mathrm{P}}^{\prime \prime}\right) \leq \gamma^{\prime}$ and, thus, $\mathcal{L}_{\mathbf{P}}(q) \leq \gamma^{\prime}<\gamma$, which is a contradiction.

For the other direction, suppose that there is a pair $\left(\sigma_{\mathrm{P}}, q\right)$ that is a timed winning strategy in $\mathrm{G}\left[q, \gamma^{\prime}\right]$ for any $\gamma^{\prime}$ such that $\gamma \leq \gamma^{\prime}<\Gamma$, but there is no timed winning strategy for $\mathbf{P}$ in $\mathbf{G}\left[q, \gamma^{\prime}\right]$ for any $\gamma^{\prime}<\gamma$. Now, $l\left(q, \sigma_{\mathrm{P}}\right)=\gamma$, and for any other strategy $\sigma_{\mathrm{P}}^{\prime}$, we have either that $l\left(q, \sigma_{\mathrm{P}}^{\prime}\right)=$ lose or that $l\left(q, \sigma_{\mathrm{p}}^{\prime}\right) \geq \gamma$. Hence, the smallest ordinal value for the strategy labels at $q$ is the ordinal $\gamma$; thus, we have that $\mathcal{L}_{\mathrm{P}}(q)=\gamma$.

(ii) If $\mathcal{L}_{\mathrm{P}}(q)=$ lose, then $l\left(q, \sigma_{\mathrm{P}}\right)=$ lose for every strategy $\sigma_{\mathrm{P}}$ of $\mathbf{P}$. Hence, none of the strategytimer pairs $\left(\sigma_{\mathrm{P}}, t\right)$ is a timed winning strategy for $\mathbf{P}$ in $\mathrm{G}[q, \gamma]$ for any $\gamma<\Gamma$. Conversely, if there is no timed winning strategy $\left(\sigma_{\mathrm{P}}, t\right)$ in $\mathrm{G}[q, \gamma]$ for any $\gamma<\Gamma$, then we have that $l\left(q, \sigma_{\mathrm{P}}\right)=$ lose for every $\sigma_{\mathrm{P}}$ and, thus, $\mathcal{L}_{\mathrm{P}}(q)=$ lose.

2. We now consider the case in which $\mathrm{P} \neq \mathrm{C}$.

(i) If $\mathcal{L}_{\mathrm{P}}(q)=$ win, then $l\left(q, \sigma_{\mathrm{P}}\right)=$ win for some strategy $\sigma_{\mathrm{P}}$, that is, $\sigma_{\mathrm{P}}$ is a winning strategy in $\mathrm{G}[q, \gamma]$ for any time limit $\gamma<\Gamma$. Conversely, if there is some $\sigma_{\mathrm{P}}$ that is a winning strategy in $\mathrm{G}[q, \gamma]$ for every time limit $\gamma<\Gamma$, then $l\left(q, \sigma_{\mathrm{P}}\right)=$ win and, thus, $\mathcal{L}_{\mathrm{P}}(q)=$ win.

(ii) Suppose that $\mathcal{L}_{\mathbf{P}}\left(q_{0}\right)=\gamma<\Gamma$, that is, $\gamma$ is the supremum of the strategy labels $l\left(q, \sigma_{\mathrm{P}}\right)$. Suppose first that there is some $\sigma_{\mathrm{P}}$ for which $l\left(q_{0}, \sigma_{\mathrm{P}}\right)=\gamma$. Now, $\sigma_{\mathrm{P}}$ is a winning strategy in $\mathrm{G}\left[q, \gamma^{\prime}\right]$ for any $\gamma^{\prime}<\gamma$. Suppose, then, that there is no maximum value for the labels $l\left(q, \sigma_{\mathrm{P}}\right)$, whence $\gamma$ must be a limit ordinal. Let $\gamma^{\prime}<\gamma$. Since $\gamma$ is the least upper bound for the strategy labels $l\left(q, \sigma_{\mathrm{P}}\right)$, there must be some strategy $\sigma_{\mathrm{P}}^{\prime}$ for which $l\left(q, \sigma_{\mathrm{P}}^{\prime}\right) \geq \gamma^{\prime}+1$, as otherwise $\gamma^{\prime}+1$ would be a lower upper bound for the strategy labels. We now observe that $\sigma_{\mathrm{P}}^{\prime}$ is a winning strategy in $\mathrm{G}\left[q, \gamma^{\prime}\right]$.

If there existed a winning strategy $\sigma_{\mathrm{P}}^{\prime}$ for $\mathbf{P}$ in $\mathrm{G}\left[q, \gamma^{\prime}\right]$ for some $\gamma^{\prime} \geq \gamma$, then we would have that $l\left(q, \sigma_{\mathrm{P}}^{\prime}\right)>\gamma$ and, thus, $\mathcal{L}_{\mathbf{P}}(q)>\gamma$. Hence, there cannot be any winning strategy for $\mathbf{P}$ in $\mathbf{G}\left[q, \gamma^{\prime}\right]$ for any $\gamma^{\prime}$ such that $\gamma \leq \gamma^{\prime}<\Gamma$.

For the other direction, assume that for every $\gamma^{\prime}<\gamma$, there exists a winning strategy for $\mathbf{P}$ in $\mathrm{G}\left[q, \gamma^{\prime}\right]$, but there exists no winning strategy for $\mathrm{P}$ in $\mathrm{G}\left[q, \gamma^{\prime}\right]$ for any $\gamma^{\prime}$ such that $\gamma \leq \gamma^{\prime}<\Gamma$. If we had that $\mathcal{L}_{\mathbf{P}}(q)=$ win, we would end up with a contradiction by the (already proved) result concerning the label win. If we had that $\gamma<\mathcal{L}_{\mathrm{P}}\left(q_{0}\right)<\Gamma$, then, by the (already proved) other direction of the current claim, there would have existed a winning strategy for P in $\mathrm{G}[q, \gamma]$, which is a contradiction. If we had $\mathcal{L}_{\mathrm{P}}(q)<\gamma$, then, once again by the other direction of the current claim, there would not have been any winning strategy for $\mathrm{P}$ in $\mathrm{G}\left[q, \mathcal{L}_{\mathrm{P}}(q)\right]$, again a contradiction. Hence, the only possibility left is that $\mathcal{L}_{\mathbf{P}}\left(q_{0}\right)=\gamma$.

\section{Proof of Proposition 4.6}

In order to simplify this proof, we will use the determinacy of $\Gamma$-bounded evaluation games (Proposition 4.15). By observing our proofs, we can confirm that this does not lead to any circular 
argumentation. This is not surprising, as Proposition 4.15 is proven for an arbitrary time limit bound $\Gamma>0$ without any assumptions on its stability. The claim of the current proposition (4.6) is needed only for showing that certain time limit bounds are guaranteed to be stable (Propositions 4.20 and 4.21). Note that by assuming the determinacy of bounded evaluation games, a player wins a bounded embedded game if and only if the player has a winning strategy in the bounded evaluation game that is continued (recall Definition 4.1).

Let $\mathrm{G}$ be an (unbounded) embedded game that can occur within $\mathcal{G}\left(\mathcal{M}, q_{i n}, \varphi\right)$. Now, the corresponding bounded embedded games $\mathrm{G}[\gamma]$ (for different time limits $\gamma$ ) can occur within $\mathcal{G}_{\Gamma}$ and $\mathcal{G}_{\Gamma^{\prime}}$. We need to show that the winning time labels for player $\mathbf{P}$ in $\mathbf{G}$ are the same for all $q \in$ St with respect to both $\Gamma$ and $\Gamma^{\prime}$. We first show that any exit position of $\mathrm{G}$ is a winning position for $\mathbf{P}$ in $\mathcal{G}_{\Gamma}$ if and only if it is a winning position for $\mathrm{P}$ in $\mathcal{G}_{\Gamma^{\prime}}$ (note that from this equivalence, it follows that $\mathrm{G}$ has the same winning condition with respect to both $\Gamma$ and $\Gamma^{\prime}$ ).

Let $\mathrm{Pos}=\left(\mathrm{P}^{\prime}, q, \psi\right)$ be a possible exit position of $\mathrm{G}$. Suppose first that Pos is a winning position for $\mathbf{P}$ in $\mathcal{G}_{\Gamma^{\prime}}$. By Proposition 4.4, the values of the winning time labels for the controller determine the lowest sufficiently large time limit for winning the corresponding embedded game-if there is one. We may thus assume that, within the player's winning strategy from Pos in $\mathcal{G}_{\Gamma^{\prime}}$, the player $\mathbf{P}$ always selects time limits corresponding to one's winning time labels for the initial positions of the embedded games. Let $\Sigma_{\mathbf{P}}$ denote such a strategy of $\mathbf{P}$ for $\mathcal{G}_{\Gamma^{\prime}}$. Since, by the assumption, all of the winning time labels of $\mathbf{P}$ are below the time limit bound $\Gamma$, it is possible for $\mathbf{P}$ to follow $\Sigma_{\mathbf{P}}$ from Pos also within $\mathcal{G}_{\Gamma}$ (note that the rules for $\mathcal{G}_{\Gamma}$ and in $\mathcal{G}_{\Gamma^{\prime}}$ differ only when a time limit for an embedded game is chosen). It is now easy to see that $\Sigma_{\mathbf{P}}$ is a winning strategy from Pos in $\mathcal{G}_{\Gamma}$.

Suppose, then, that Pos is not a winning position for $\mathbf{P}$ in $\mathcal{G}_{\Gamma^{\prime}}$. By the determinacy of bounded embedded games, Pos must be a winning position for $\overline{\mathbf{P}}$ in $\mathcal{G}_{\Gamma^{\prime}}$. Hence, we can argue exactly as above, that Pos is a winning position for $\overline{\mathbf{P}}$ in $\mathcal{G}_{\Gamma}$ and, thus, it cannot be a winning position for $\mathrm{P}$ in $\mathcal{G}_{\Gamma}$. We can thus conclude that Pos is a winning position for $\mathrm{P}$ in $\mathcal{G}_{\Gamma}$ if and only it is a winning position for $\mathbf{P}$ in $\mathcal{G}_{\Gamma^{\prime}}$.

By the equivalence that was proven above, it is easy to see by Definition 4.2, that the bounds $\Gamma$ and $\Gamma^{\prime}$ cannot create two different ordinal valued winning time labels for $\mathbf{P}$ in $\mathbf{G}$. The remaining possibility is that there is a state $q \in \mathrm{St}$ that has a label lose/win with the time limit bound $\Gamma$ and some ordinal value $\gamma$ with the time limit bound $\Gamma^{\prime}$. But, again, by Definition 4.2 , we see that this is possible only if $\gamma \geq \Gamma$, which would contradict the assumption that the winning time labels of $\mathrm{P}$ in $\mathrm{G}$ with respect to $\Gamma^{\prime}$ are all strictly below $\Gamma$. This concludes the proof.

\section{Proof of Proposition 4.10}

1. We first consider the case in which $\mathbf{P}=\mathbf{C}$.

We will prove by transfinite induction on $\gamma<\Gamma$ that for every $q \in$ St, if $\mathbf{P}$ has a timed winning strategy in $\mathrm{G}[q, \gamma]$, then $\left(\tau_{\mathrm{P}}, t_{\text {can }}\right)$ is a timed winning strategy in $\mathrm{G}[q, \gamma]$. Let the inductive hypothesis be that the claim holds for every $\gamma^{\prime}<\gamma$ and suppose that $\mathbf{P}$ has a timed winning strategy in $\mathrm{G}[q, \gamma]$. By Proposition 4.4, we have that $\mathcal{L}_{\mathrm{P}}(q)=\delta$ for some $\delta \leq \gamma$. Let $\sigma_{\mathrm{P}}^{\prime}$ be a strategy for which $\tau_{\mathrm{P}}(q)=\sigma_{\mathrm{P}}^{\prime}(\delta, q)$ and there is a timer $t^{\prime}$ such that $\left(\sigma_{\mathrm{P}}^{\prime}, t^{\prime}\right)$ is a timed winning strategy in $\mathrm{G}\left[q, \delta^{\prime}\right]$ for all $\delta^{\prime}$ such that $\delta \leq \delta^{\prime}<\Gamma$ (such a strategy exists by the definition of the canonical strategy $\tau_{\mathrm{P}}$ ).

Suppose first that $\delta=0$, whence $\left(\sigma_{\mathrm{P}}^{\prime}, t^{\prime}\right)$ is a timed winning strategy in $\mathrm{G}[q, 0]$. Now, we have that $\tau_{\mathbf{P}}(q)=\sigma_{\mathbf{P}}^{\prime}(0, q)=\psi_{\mathrm{C}}$ and, thus, $\left(\tau_{\mathbf{P}}, t_{\text {can }}\right)$ is a timed winning strategy in $\mathrm{G}[q, \gamma]$. Suppose, then, that $\delta>0$, whence $\sigma_{\mathrm{P}}^{\prime}(\delta, q)$ must be either some tuple of actions for the coalition $A$ or some response function for the coalition $\bar{A}$. Let $Q \subseteq$ St be the set of states that is forced by $\sigma_{\mathrm{P}}^{\prime}(\delta, q)$ and let $q^{\prime} \in Q$. Since $\left(\sigma_{\mathrm{P}}^{\prime}, t^{\prime}\right)$ is a timed winning strategy in $\mathrm{G}[q, \delta]$, there is that $\delta^{\prime}<\delta$ such that $\left(\sigma_{\mathrm{P}}^{\prime}, t^{\prime}\right)$ 
is a timed winning strategy in $\mathrm{G}\left[q^{\prime}, \delta^{\prime}\right]$ (if $\delta$ is a limit ordinal, then $\delta^{\prime}=t^{\prime}\left(\delta, q^{\prime}\right)$; else, $\delta^{\prime}=\delta-1$ ). Since $\delta \leq \gamma$, we have that $\delta^{\prime}<\gamma$.

For the inductive step, suppose first that $\gamma$ is a successor ordinal. Since we have that $\delta^{\prime} \leq \gamma-1$, there is a timed winning strategy $\left(\sigma_{\mathrm{p}}^{\prime \prime}, t^{\prime \prime}\right)$ in $\mathrm{G}\left[q^{\prime}, \gamma-1\right]$ by Lemma 4.3 . Thus, by the inductive hypothesis, $\left(\tau_{\mathrm{P}}, t_{\text {can }}\right)$ is a timed winning strategy in $\mathrm{G}\left[q^{\prime}, \gamma-1\right]$. Since this holds for every $q^{\prime} \in Q$, we see that $\left(\tau_{\mathrm{P}}, t_{c a n}\right)$ is a timed winning strategy in $\mathrm{G}[q, \gamma]$.

Suppose now that $\gamma$ is a limit ordinal. Since $\left(\sigma_{\mathrm{P}}^{\prime}, t^{\prime}\right)$ is a timed winning strategy in $\mathrm{G}\left[q^{\prime}, \delta^{\prime}\right]$, by Proposition 4.4, we must have that $\mathcal{L}_{\mathrm{P}}\left(q^{\prime}\right) \leq \delta^{\prime}<\gamma$. Thus, by the definition of the canonical timer, $t_{\text {can }}\left(\gamma, q^{\prime}\right)=\mathcal{L}_{\mathbf{P}}\left(q^{\prime}\right)$. By Proposition 4.4 , there is a timed winning strategy $\left(\sigma_{\mathrm{P}}^{\prime \prime}, t^{\prime \prime}\right)$ in $\mathrm{G}\left[q^{\prime}, \mathcal{L}_{\mathbf{P}}\left(q^{\prime}\right)\right]$. Thus, by the inductive hypothesis, $\left(\tau_{\mathrm{P}}, t_{\text {can }}\right)$ is a timed winning strategy in $\mathrm{G}\left[q^{\prime}, \mathcal{L}_{\mathrm{P}}\left(q^{\prime}\right)\right]$. Since this holds for every $q^{\prime} \in Q$, and $t_{c a n}\left(\gamma, q^{\prime}\right)=\mathcal{L}_{\mathbf{P}}\left(q^{\prime}\right)$ for every $q^{\prime} \in Q$, we see that $\left(\tau_{\mathrm{P}}, t_{\text {can }}\right)$ is a timed winning strategy in $\mathrm{G}[q, \gamma]$.

2. We then consider the case in which $\mathbf{P} \neq \mathbf{C}$.

We will prove by transfinite induction on $\gamma<\Gamma$ that for every $q \in Q$ : if $\mathbf{P}$ has a winning strategy in $\mathrm{G}[q, \gamma]$, then $\tau_{\mathrm{P}}$ is a winning strategy in $\mathrm{G}[q, \gamma]$.

Suppose first that $\gamma=0$ and that $\mathbf{P}$ has a winning strategy $\sigma_{\mathbf{P}}$ in $\mathbf{G}[q, 0]$. Now, since with the time limit 0 the game will end at $q$ immediately, every strategy of $\mathbf{P}$ will be a winning strategy in $\mathrm{G}[q, 0]$. Hence, in particular, $\tau_{\mathrm{P}}$ is a winning strategy in $\mathrm{G}[q, 0]$.

For the inductive step, suppose that the claim holds for every $\gamma^{\prime}<\gamma$ and that $\mathbf{P}$ has a winning strategy $\sigma_{\mathrm{P}}$ in $\mathrm{G}[q, \gamma]$. By Proposition 4.4 , we have either that $\mathcal{L}_{\mathrm{P}}(q)=$ win or that $\mathcal{L}_{\mathrm{P}}(q)>\gamma$.

Assume first that $\mathcal{L}_{\mathrm{P}}(q)=$ win. Let $\sigma_{\mathrm{P}}$ be a strategy for which $l\left(q, \sigma_{\mathrm{P}}\right)=$ win and $\tau_{\mathrm{P}}(\gamma, q)=$ $\sigma_{\mathrm{P}}(\gamma, q)$ (such a strategy exists by the definition of $\tau_{\mathrm{P}}$ ). Let $Q \subseteq$ St be the set of states forced by $\sigma_{\mathrm{P}}(\gamma, q)$ and let $q^{\prime} \in Q$. Since $l\left(q, \sigma_{\mathrm{P}}\right)=$ win, the strategy $\sigma_{\mathrm{P}}$ is a winning strategy in $\mathrm{G}[q, \delta]$ for every $\delta<\Gamma$; therefore, as we have that $\gamma<\Gamma$, the strategy $\sigma_{\mathrm{P}}$ must also be a winning strategy in $\mathrm{G}\left[q^{\prime}, \gamma^{\prime}\right]$ for every $\gamma^{\prime}<\gamma$. Thus, there is a winning strategy in $\mathrm{G}\left[q^{\prime}, \gamma^{\prime}\right]$ for every $\gamma^{\prime}<\gamma$ and every $q^{\prime} \in Q$. Hence, by the inductive hypothesis, $\tau_{\mathrm{P}}$ is a winning strategy in $\mathrm{G}\left[q^{\prime}, \gamma^{\prime}\right]$ for every $\gamma^{\prime}<\gamma$ and $q^{\prime} \in Q$. Therefore, we observe that $\tau_{\mathrm{P}}$ is also a winning strategy in $\mathrm{G}[q, \gamma]$.

Assume now that $\mathcal{L}_{\mathrm{P}}(q)=\delta>\gamma$. Let $\sigma_{\mathrm{P}}$ be a strategy for which $l\left(q, \sigma_{\mathrm{P}}\right)>\gamma$ and $\tau_{\mathrm{P}}(\gamma, q)=$ $\sigma_{\mathrm{P}}(\gamma, q)$ (such a strategy exists by the definition of $\tau_{\mathrm{P}}$ ). Let $Q \subseteq$ St be the set of states that is forced by $\sigma_{\mathrm{P}}(\gamma, q)$ and let $q^{\prime} \in Q$. Since $\sigma_{\mathrm{P}}$ is a winning strategy in $\mathrm{G}\left[q, \delta^{\prime}\right]$ for every $\delta^{\prime}<l\left(q, \sigma_{\mathrm{P}}\right)$ and since we have that $\gamma<l\left(q, \sigma_{\mathrm{P}}\right)$, the strategy $\sigma_{\mathrm{P}}$ must also be a winning strategy in $\mathrm{G}\left[q^{\prime}, \gamma^{\prime}\right]$ for every $\gamma^{\prime}<\gamma$. Hence, we can deduce, as before, that $\tau_{\mathrm{P}}$ is a winning strategy in the games for the configurations over $Q$ that follow $(\gamma, q)$; thus, $\tau_{\mathrm{P}}$ is also a winning strategy in $\mathrm{G}[q, \gamma]$.

\section{Proof of Proposition 4.13}

We prove this claim by transfinite induction on the ordinal $\gamma<\Gamma$. We first prove the special case in which $\gamma=0$. Let $q \in$ St and suppose first that $\mathcal{L}_{\mathrm{C}}(q)=0$, whence, by Proposition 4.4, the player $\mathrm{C}$ has a timed winning strategy $\left(\sigma_{\mathbf{C}}, t\right)$ in $\mathrm{G}[q, 0]$. This is possible only if the exit position $\left(\mathbf{C}, q, \psi_{\mathbf{C}}\right)$ is a winning position for $\mathrm{C}$. In that case, $\overline{\mathrm{C}}$ loses $\mathrm{G}\left[q, \gamma^{\prime}\right]$ with any time limit $\gamma^{\prime}$ and, thus, $\mathcal{L}_{\overline{\mathrm{C}}}(q)=0$. Suppose, then, that $\mathcal{L}_{\overline{\mathrm{C}}}(q)=0$, whence there is no winning strategy for $\overline{\mathrm{C}}$ in $\mathrm{G}[q, 0]$. This is possible only if $\mathrm{C}$ wins at the exit position $\left(\mathrm{C}, q, \psi_{\mathrm{C}}\right)$; whence, $\mathcal{L}_{\mathrm{C}}(q)=0$.

Now, let $\gamma>0$. The inductive hypothesis is that the claim holds for every ordinal $\gamma^{\prime}<\gamma$. Suppose first that $\mathcal{L}_{\mathrm{C}}(q)=\gamma$. By Proposition 4.10, there is a timed winning strategy $\left(\sigma_{\mathrm{P}}, t\right)$ in $\mathrm{G}[q, \gamma]$. Hence, $\overline{\mathrm{C}}$ cannot have a winning strategy in $\mathrm{G}[q, \gamma]$; thus, by Proposition 4.4, we must have that $\mathcal{L}_{\overline{\mathrm{C}}}(q) \leq \gamma$. If $\mathcal{L}_{\overline{\mathrm{C}}}(q)<\gamma$, then, by the inductive hypothesis, we have that $\mathcal{L}_{\mathrm{C}}(q)=\mathcal{L}_{\overline{\mathrm{C}}}(q)<\gamma$, which is a contradiction. Therefore, it must be that $\mathcal{L}_{\overline{\mathrm{C}}}(q)=\gamma$. 
Suppose, then, that $\mathcal{L}_{\overline{\mathrm{C}}}(q)=\gamma$. We will next show that for any strategy $\sigma_{\overline{\mathrm{C}}}$ and any set $Q \subseteq \mathrm{St}$ forced by $\sigma_{\overline{\mathrm{C}}}(\gamma, q)$, there is a state $q^{\prime} \in Q$ for which $\mathcal{L}_{\overline{\mathrm{C}}}\left(q^{\prime}\right)<\gamma$. For the sake of contradiction, suppose that there is a strategy $\sigma_{\overline{\mathrm{C}}}$ such that for the set $Q \subseteq$ St forced by $\sigma_{\overline{\mathrm{C}}}(\gamma, q)$, we have that $\mathcal{L}_{\overline{\mathrm{C}}}\left(q^{\prime}\right) \geq \gamma$ or $\mathcal{L}_{\overline{\mathrm{C}}}\left(q^{\prime}\right)=$ win for every $q^{\prime} \in Q$. We construct the following strategy $\sigma_{\overline{\mathrm{C}}}^{\prime}$ for $\overline{\mathrm{C}}$ in the embedded game $\mathrm{G}[q, \gamma]$ :

$$
\begin{aligned}
& \sigma_{\overline{\mathrm{C}}}^{\prime}(\delta, q)=\sigma_{\overline{\mathrm{C}}}(\gamma, q) \text { for every } \delta \leq \gamma, \\
& \sigma_{\overline{\mathrm{C}}}^{\prime}\left(\delta, q^{\prime}\right)=\tau_{\overline{\mathrm{C}}}\left(\delta, q^{\prime}\right) \text { for every } \delta \leq \gamma, \text { and } q^{\prime} \in \mathrm{St} \backslash\{q\} .
\end{aligned}
$$

Since $\mathcal{L}_{\overline{\mathrm{C}}}\left(q^{\prime}\right) \geq \gamma$ or $\mathcal{L}_{\overline{\mathrm{C}}}\left(q^{\prime}\right)=$ win for every $q^{\prime} \in Q$, by Proposition 4 .4, the canonical strategy $\tau_{\overline{\mathrm{C}}}$ is a winning strategy in $\mathrm{G}\left[q^{\prime}, \delta\right]$ for any $q^{\prime} \in Q$ and $\delta<\gamma$. Thus, it is easy to see that $\sigma_{\overline{\mathrm{C}}}^{\prime}$ is a winning strategy in $\mathrm{G}[q, \gamma]$. Hence, again by Proposition 4.4 , we must have that $\mathcal{L}_{\overline{\mathrm{C}}}(q)>\gamma$ or $\mathcal{L}_{\overline{\mathrm{C}}}(q)=$ win, which is a contradiction. Therefore, we infer the following:

For any strategy $\sigma_{\overline{\mathrm{C}}}$ and $Q \subseteq$ St forced by $\sigma_{\overline{\mathrm{C}}}(\gamma, q)$,

there is some $q^{\prime} \in Q$ such that $\mathcal{L}_{\overline{\mathrm{C}}}\left(q^{\prime}\right)<\gamma$.

Let $Q^{\prime}:=\left\{q^{\prime} \in \mathrm{St} \mid \mathcal{L}_{\overline{\mathrm{C}}}\left(q^{\prime}\right)<\gamma\right\}$. By the inductive hypothesis, $\mathcal{L}_{\mathrm{C}}\left(q^{\prime}\right)<\gamma$ for every $q^{\prime} \in Q^{\prime}$. We will show that $\mathrm{C}$ can play in such a way at $q$ that all possible successor states will be in the set $Q^{\prime}$. Suppose first that $\mathrm{C}=\overline{\mathrm{V}}$. Since for every $\vec{\alpha} \in \operatorname{action}(q, A)$, there is some strategy $\sigma_{\overline{\mathrm{C}}}$ such that $\sigma_{\overline{\mathrm{C}}}(\gamma, q)=\vec{\alpha}$, we infer by $(\star)$ that there is some response function for $\bar{A}$ that forces the next state to be in $Q^{\prime}$. Suppose, then, that $\mathrm{C}=\mathrm{V}$. If for every $\vec{\alpha} \in \operatorname{action}(q, A)$ there existed some $\vec{\beta} \in \operatorname{action}(q, \bar{A})$ such that the outcome state of these actions was not in $Q^{\prime}$, then there would be some strategy $\sigma_{\overline{\mathrm{C}}}$ such that the set forced by $\sigma_{\overline{\mathrm{C}}}(\gamma, q)$ would not intersect $Q^{\prime}$. This is a contradiction with $(\star)$; thus, there is some tuple of actions for $A$ at $q$ such that all possible successor states will be in $Q^{\prime}$.

We next construct a strategy $\sigma_{\mathrm{C}}$ for $\mathrm{C}$ in $\mathrm{G}[q, \gamma]$. By the description above, we can define $\sigma_{\mathrm{C}}$ for every configuration $\left(\gamma^{\prime}, q\right)$, where $\gamma^{\prime} \leq \gamma$, in such a way that the set forced by $\sigma_{\mathrm{C}}\left(\gamma^{\prime}, q\right)$ is a subset of $Q^{\prime}$. For all configurations $\left(\gamma^{\prime}, q^{\prime}\right)$, where $\gamma^{\prime} \leq \gamma$ and $q^{\prime} \in$ St $\backslash\{q\}$, we define $\sigma_{\mathrm{C}}\left(\gamma^{\prime}, q^{\prime}\right)=\tau_{\mathrm{C}}\left(q^{\prime}\right)$. Since $\mathcal{L}_{\mathrm{C}}\left(q^{\prime}\right)<\gamma$ for every $q^{\prime} \in Q^{\prime}$, we infer that $t_{\text {can }}\left(\gamma, q^{\prime}\right)=\mathcal{L}_{\mathrm{C}}\left(q^{\prime}\right)<\gamma$ for every $q^{\prime} \in Q$. By Propositions 4.4 and $4.10,\left(\tau_{\mathrm{C}}, t_{\text {can }}\right)$ is a timed winning strategy in $\mathrm{G}\left[q^{\prime}, t_{\text {can }}\left(\gamma, q^{\prime}\right)\right]$ for any $q^{\prime} \in Q^{\prime}$. Thus, it is easy to see that $\left(\sigma_{\mathrm{C}}, t_{\text {can }}\right)$ is a timed winning strategy in $\mathrm{G}[q, \gamma]$. However, since $\mathcal{L}_{\overline{\mathrm{C}}}(q)=$ $\gamma$, we conclude, using the inductive hypothesis, that there cannot be a timed winning strategy for $\mathrm{C}$ in $\mathrm{G}\left[q, \gamma^{\prime}\right]$ for any $\gamma^{\prime}<\gamma$. Hence, by Proposition 4.4, we must have that $\mathcal{L}_{\mathrm{C}}(q)=\gamma$.

\section{Proof of Proposition 4.15}

Since both of the players cannot have a winning strategy, the implication from left to right is immediate. We prove the implication from right to left by induction on $\varphi$.

- Suppose that $\varphi=p$ (where $p \in \Pi$ ). By the rules of the game, it is easy to see that each ending position is a winning position for either of the players. The claim follows from this.

-Suppose that $\varphi=\neg \psi$. Since negation just swaps the verifier and falsifier for the position, this case follows directly from the inductive hypothesis.

- Suppose that $\varphi=\psi \vee \theta$. Suppose that $\overline{\mathbf{P}}$ does not have a winning strategy in $\mathcal{G}(\mathcal{M}, q, \psi \vee$ $\theta)$. Suppose first that $\overline{\mathbf{P}}=\mathbf{A}$, whence it is not possible that both $(\mathbf{E}, q, \psi)$ and $(\mathbf{E}, q, \theta)$ are winning positions for $\overline{\mathbf{P}}$. Thus, by the inductive hypothesis, at least one of these positions must be a winning position for $\mathbf{E}=\mathbf{P}$; thus, $\mathbf{P}$ has a winning strategy in $\mathcal{G}(\mathcal{M}, q, \psi \vee \theta)$. Suppose, then, that $\overline{\mathbf{P}}=\mathbf{E}$, whence neither position $(\mathbf{E}, q, \psi)$ nor position $(\mathbf{E}, q, \theta)$ can be a winning position for $\overline{\mathbf{P}}$. Thus, by the inductive hypothesis, both of these positions must be winning positions for $\mathrm{A}=\mathbf{P}$; thus, $\mathbf{P}$ has a winning strategy in $\mathcal{G}(\mathcal{M}, q, \psi \vee \theta)$. 
- Suppose that $\varphi=\langle\langle A\rangle\rangle \mathrm{X} \psi$. This case can be proven by an analogous argument as the one used in the inductive step of Proposition 4.13. Note that the rule related to the position $(\mathbf{E}, q,\langle\langle A\rangle\rangle \mathrm{X} \psi)$ is just a special case of the bounded embedded game $\mathbf{g}(\mathbf{E}, \mathbf{E}, A, q, \psi, \perp)[1]$ with the restriction that neither of the players may end the game before time runs out.

- Suppose that $\varphi=\langle\langle A\rangle\rangle \psi \cup \theta$. Let $\mathrm{G}$ be the embedded game that arises from $\varphi$. From the inductive hypothesis, it follows that the player who has a winning strategy in $\mathrm{G}$ also has a winning strategy in the evaluation game that is continued (recall Definition 4.1).

Suppose first that $\overline{\mathbf{P}}=\mathrm{A}$, whence there must be (at least one) $\gamma<\Gamma$ such that $\overline{\mathbf{P}}$ does not have a winning strategy in $\mathrm{G}$ with the time limit $\gamma$. Since bounded embedded games are determined (by Corollary 4.14), it follows from the inductive hypothesis that $\mathbf{E}=\mathbf{P}$ must have a (timed) winning strategy in $\mathrm{G}[\gamma]$. Hence, $\mathrm{P}$ has a winning strategy in $\mathcal{G}(\mathcal{M}, q,\langle\langle A\rangle \psi \cup \theta)$ (P first chooses the time limit to be $\gamma$ and then follows her timed winning strategy in $G[\gamma]$ ).

Suppose, then, that $\overline{\mathbf{P}}=\mathrm{E}$, whence $\overline{\mathrm{P}}$ cannot have a (timed) winning strategy in G with any time limit $\gamma<\Gamma$. Since bounded embedded games are determined (by Corollary 4.14), it follows from the inductive hypothesis that $\mathbf{A}=\mathbf{P}$ must have a winning strategy in $\mathrm{G}[\gamma]$ for every $\gamma<\Gamma$. Hence, $\mathbf{P}$ has a winning strategy in $\mathcal{G}(\mathcal{M}, q,\langle\langle A\rangle \psi \cup \theta)(\operatorname{after} \overline{\mathbf{P}}$ has chosen a time limit $\gamma$, then $\mathbf{P}$ just follows his winning strategy in $\mathrm{G}[\gamma]$ ).

- The case $\varphi=\langle\langle A\rangle\rangle \mathrm{R} \theta$ is proven dually to the case $\varphi=\langle\langle A\rangle\rangle \psi \mathrm{U} \theta$.

\section{Proof of Lemma 4.18}

Suppose that $\mathcal{L}_{\mathrm{P}}(q)=\gamma>0$. Since $\mathcal{L}_{\mathrm{P}}(q) \neq 0$, canonically timed strategy $\left(\tau_{\mathrm{P}}, t_{\text {can }}\right)$ is not a timed winning strategy for $\mathbf{P}$ in $\mathrm{G}[q, 0]$. Therefore $\tau_{\mathrm{P}}(q)$ is either some tuple of actions for $A$ or some response function for $\bar{A}$. Let $Q \subseteq$ St be the set of states that is forced by $\tau_{\mathrm{P}}(q)$. We first show that $\mathcal{L}_{\mathrm{P}}\left(q^{\prime}\right)<\gamma$ for every $q^{\prime} \in Q$. Since $\left(\tau_{\mathrm{P}}, t_{c a n}\right)$ is a timed winning strategy in $\mathrm{G}[q, \gamma]$, it must also be a timed winning strategy in $\mathrm{G}\left[q^{\prime}, t_{\text {can }}\left(\gamma, q^{\prime}\right)\right]$ for every $q^{\prime} \in Q$. Hence, by the definition of canonical timer, $\gamma>t_{\text {can }}\left(\gamma, q^{\prime}\right)=\mathcal{L}_{\mathbf{P}}\left(q^{\prime}\right)$ for every $q^{\prime} \in Q$.

Suppose first that $\gamma$ is a successor ordinal. If we would have that $\mathcal{L}_{\mathbf{P}}\left(q^{\prime}\right)<\gamma-1$ for every $q^{\prime} \in Q$, then $\left(\tau_{\mathrm{P}}, t_{\text {can }}\right)$ would be a winning strategy in $\mathrm{G}[q, \gamma-1]$ and, thus, we would have that $\mathcal{L}_{\mathrm{P}}(q) \leq$ $\gamma-1$. Hence, $\gamma>\mathcal{L}_{\mathbf{P}}\left(q^{\prime}\right) \geq \gamma-1$ for some $q^{\prime} \in Q$ and, thus, $\max \left\{\mathcal{L}_{\mathbf{P}}\left(q^{\prime}\right) \mid q^{\prime} \in Q\right\}=\gamma-1$.

Suppose, then, that $\gamma$ is a limit ordinal. If $\gamma^{\prime}<\gamma$ would be an upper bound for the winning time labels in $Q$, then we would have that $\mathcal{L}_{\mathrm{P}}\left(q^{\prime}\right)<\gamma^{\prime}+1$ for every $q^{\prime} \in Q$. Hence, $\left(\tau_{\mathrm{P}}, t_{\text {can }}\right)$ would be a timed winning strategy in $\mathrm{G}\left[q, \gamma^{\prime}+1\right]$ and, thus, we would have that $\mathcal{L}_{\mathrm{P}}(q) \leq \gamma^{\prime}+1<\gamma$. This is impossible; thus, $\sup \left\{\mathcal{L}_{\mathrm{P}}\left(q^{\prime}\right) \mid q^{\prime} \in Q\right\}=\gamma$.

\section{Proof of Theorem 5.1}

We show by induction on $\varphi$ that the following equivalence holds for every $q \in$ St.

$$
\mathcal{M}, q \mid \varphi \text { iff } \mathcal{M}, q \mid{ }^{g}{ }_{u} \varphi .
$$

- Suppose that $\varphi=p(p \in \Pi)$. This case is trivial since the winning condition for the ending position $(\mathrm{E}, q, p)$ corresponds to the truth condition for $p$ in the compositional semantics.

- Suppose that $\varphi=\neg \psi$.

Suppose first that $\mathcal{M}, q \vDash \neg \psi$, that is, $\mathcal{M}, q \vDash \psi$. Therefore, by the inductive hypothesis, $\mathcal{M}, q \nvdash_{u}^{g} \psi$. Since, by Proposition 4.15, evaluation games are determined, (E, $\left.q, \psi\right)$ is a winning position for Abelard. Therefore, by the rule for negation, $(\mathbf{A}, q, \neg \psi)$ is also a winning position for Abelard. Since all the rules of the game for the players are symmetric, dually $(\mathrm{E}, q, \neg \psi)$ is a winning position for Eloise; thus, $\mathcal{M}, q \mid{ }^{g}{ }_{u}^{g} \neg \psi$. 
Suppose, then, that $\mathcal{M},\left.q\right|^{g}{ }_{u}^{g} \neg \psi$. Now, by the rule for negation, (A, $\left.q, \psi\right)$ is a winning position for Eloise and, thus, dually $(\mathrm{E}, q, \psi)$ is a winning position for Abelard. However, since only one of the players can have a winning strategy from the position (E, $q, \psi)$, we must have that $\mathcal{M}, q \forall_{u}^{g} \psi$. Hence, by the inductive hypothesis, $\mathcal{M}, q \notin \psi$, that is, $\mathcal{M}, q \mid=$ $\neg \psi$.

- Suppose that $\varphi=\psi \vee \theta$.

Suppose first that $\mathcal{M}, q \mid=\psi \vee \theta$, that is, $\mathcal{M}, q=\psi$ or $\mathcal{M}, q=\theta$. If the former holds, we define $\Sigma_{\mathrm{E}}(\mathrm{E}, q, \psi \vee \theta)=\psi$; else, define $\Sigma_{\mathrm{E}}(\mathrm{E}, q, \psi \vee \theta)=\theta$. Now, by the inductive hypothesis, the next position of the game must be a winning position for Eloise; thus, $\mathcal{M},\left.q\right|^{g}{ }_{u}^{g} \psi \vee \theta$. Suppose, then, that $\mathcal{M}, q \mid=_{u}^{g} \psi \vee \theta$, that is, Eloise has a winning strategy $\Sigma_{\mathrm{E}}$ from the position $(\mathbf{E}, q, \psi \vee \theta)$. Now, $\Sigma_{\mathbf{E}}$ picks either $\psi$ or $\theta$. If $\Sigma_{\mathbf{E}}(\mathbf{E}, q, \psi \vee \theta)=\psi$, then, by the inductive hypothesis, we must have that $\mathcal{M}, q \mid \psi \psi$. If $\Sigma_{\mathbf{E}}(\mathrm{E}, q, \psi \vee \theta)=\theta$, then we analogously have $\mathcal{M}, q \mid \theta$. Therefore, $\mathcal{M}, q \mid \psi \vee \theta$.

- Suppose that $\varphi=\langle\langle A\rangle\rangle \psi$.

Suppose first that $\mathcal{M}, q \mid=\left\langle\langle A\rangle \mathrm{X} \psi\right.$, that is, there exists a collective strategy $S_{A}$ such that for each $\Lambda \in$ paths $\left(q, S_{A}\right)$, we have that $\mathcal{M}, \Lambda[1] \mid=\psi$. Let $\Sigma_{\mathrm{E}}(\mathrm{E}, q,\langle\langle A\rangle\rangle \mathrm{X} \psi)$ be the tuple in action $(A, q)$, which is determined by $S_{A}$ at $q$. Now, regardless of the actions chosen by Abelard for the agents in $\bar{A}$, the resulting state $q^{\prime}$ must be $\Lambda[1]$ for some $\Lambda \in$ paths $\left(q, S_{A}\right)$ and, thus, $\mathcal{M}, q^{\prime} \mid=\psi$. By the inductive hypothesis, $\mathcal{M}, q^{\prime} \mid{ }^{g}{ }_{u}^{g} \psi$, that is, Eloise has a winning strategy from the position $\left(\mathrm{E}, q^{\prime}, \psi\right)$. Therefore, we have that $\mathcal{M}, q \mid=_{u}^{g}\langle\langle A\rangle\rangle \mathrm{X} \psi$.

Suppose, then, that $\mathcal{M}, q \mid{ }^{g}{ }_{u}^{g}\langle A A\rangle \mathrm{X} \psi$, that is, Eloise has a winning strategy $\Sigma_{\mathrm{E}}$ from the position $\left(\mathbf{E}, q,\langle\langle A\rangle \mathrm{X} \psi)\right.$. Now, $\Sigma_{\mathbf{E}}$ assigns some tuple of choices for the agents in $A$. We can now construct a related collective strategy $S_{A}$ by using those choices at $q$; the choices at other states may be arbitrary. Let $\Lambda \in$ paths $\left(q, S_{A}\right)$. Now, Abelard can choose such actions for $\bar{A}$ that the resulting state is $\Lambda[1]$. Since $\Sigma_{\mathbf{E}}$ is a winning strategy, the position $(\mathbf{E}, \Lambda[1], \psi)$ is a winning position for Eloise. Hence, $\mathcal{M}, \Lambda[1] \mid{ }^{g}{ }_{u}^{g} \psi$ and, thus, by the inductive hypothesis, $\mathcal{M}, \Lambda[1] \mid=\psi$. Therefore, $\mathcal{M}, q \mid=\langle\langle A\rangle\rangle \mathrm{X} \psi$.

- Suppose that $\varphi=\langle\langle A\rangle \psi \cup \theta$.

Suppose first that $\mathcal{M}, q \mid\langle\langle A\rangle\rangle \psi \cup \theta$, that is, there exists $S_{A}$ such that for each $\Lambda \in$ paths $\left(q, S_{A}\right)$, there is some $i \geq 0$ such that $\mathcal{M}, \Lambda[i] \mid=\theta$ and $\mathcal{M}, \Lambda[j] \mid=\psi$ for every $j<i$. Let $\Sigma_{\mathbf{E}}\left(\mathrm{E}, q,\langle\langle A\rangle \psi \cup \theta)\right.$ be the strategy $\sigma_{\mathrm{E}}$, defined as follows: let $\sigma_{\mathrm{E}}\left(q^{\prime}\right)=\theta$ for each $q^{\prime} \in$ St, where $\mathcal{M}, q^{\prime} \mid=\theta$, and for all other states $q^{\prime} \in \mathrm{St}$, let $\sigma_{\mathrm{E}}\left(q^{\prime}\right)$ be the tuple of actions for the agents in $A$ determined by $S_{A}$. Now, regardless of the actions of Abelard, all of the states that are reached in the embedded game must be states $\Lambda[i]$ for some $\Lambda \in \operatorname{paths}\left(q, S_{A}\right)$ and $i \geq 0$. Thus, when Eloise uses $\sigma_{\mathrm{E}}$, a state $q^{\prime}$ where $\mathcal{M}, q^{\prime} \mid=\theta$ is reached in a finite number of rounds. If Abelard ends the game before that at some state $q^{\prime}$, then we have that $\mathcal{M}, q^{\prime}=\psi$. By the inductive hypothesis, either of these cases will result in an exit position that is a winning position for Eloise. Therefore, we have that $\mathcal{M}, q \models_{u}^{g}\langle\langle A\rangle \psi \cup \theta$.

Suppose, then, that $\mathcal{M},\left.q\right|^{g}{ }_{u}^{g}\left\langle\langle A\rangle \psi \cup \theta\right.$, that is, Eloise has a winning strategy $\Sigma_{\mathrm{E}}$ from the position $(\mathrm{E}, q,\langle\langle A\rangle\rangle \cup \cup \theta)$. Now, $\Sigma_{\mathbf{E}}\left(\mathbf{E}, q,\langle\langle A\rangle \psi \cup \theta)\right.$ is some strategy $\sigma_{\mathrm{E}}$ for the corresponding embedded game. We can now construct a collective strategy $S_{A}$ that is related to the strategy $\sigma_{\mathrm{E}}$ : for any state in which $\sigma_{\mathrm{E}}$ assigns some tuple of actions for agents in $A$, we define the same actions for $S_{A}$. For states in which $\sigma_{\mathrm{E}}$ instructs to end the game, we may define arbitrary actions for $S_{A}$. We will use this same method from now on, when we define collective strategies $S_{A}$ related to the strategies of $\mathbf{V}$ in an embedded game.

Let $\Lambda \in$ paths $\left(q, S_{A}\right)$. Now, when Eloise uses $\sigma_{\mathrm{E}}$, there will be actions of Abelard such that the states of the embedded game are on $\Lambda$ until some configuration $\Lambda[i]$, at which Eloise 
ends the game at the exit position $(\mathbf{E}, \Lambda[i], \theta)$. (Note that since $\Sigma_{\mathbf{E}}$ is a winning strategy, Eloise must always end the embedded game after finitely many steps.) Since $\Sigma_{\mathrm{E}}$ is a winning strategy, we must have that $\mathcal{M}, \Lambda[i] \mid=\theta$ by the inductive hypothesis. Then, let $j<i$. Now, Abelard can end the game after $j$ rounds at the position $(\mathrm{E}, \Lambda[j], \psi)$. Since that is a winning position for Eloise, we must have that $\mathcal{M}, \Lambda[j] \vDash \psi \psi \psi$ by the inductive hypothesis. Therefore, we conclude that $\mathcal{M}, q \mid\langle\langle A\rangle \psi \cup \theta$.

- Suppose that $\varphi=\langle\langle A\rangle \psi \mathrm{R} \theta$.

Suppose that $\mathcal{M}, q \mid=\left\langle\langle A\rangle \psi \mathrm{R} \theta\right.$, that is, there exists a strategy $S_{A}$ such that for each $\Lambda \in$ paths $\left(q, S_{A}\right)$ and $i \geq 0$, either $\mathcal{M}, \Lambda[i] \mid=\theta$ or there is $j<i$ such that $\mathcal{M}, \Lambda[j] \models \psi$. Let $\Sigma_{\mathrm{E}}(\mathrm{E}, q,\langle\langle A\rangle\rangle \mathrm{R} \theta)$ be the strategy $\sigma_{\mathrm{E}}$ that is defined as follows: $\sigma_{\mathrm{E}}\left(q^{\prime}\right)=\psi$ for each $q^{\prime} \in \mathrm{St}$, where $\mathcal{M}, q^{\prime} \mid=\psi$ and for all other states $q^{\prime} \in$ St, let $\sigma_{\mathrm{E}}\left(q^{\prime}\right)$ be the tuple of actions for the agents in $A$ determined by $S_{A}$. Now, all states that are reached in the embedded game must be states $\Lambda[i]$ for some $\Lambda \in$ paths $\left(q, S_{A}\right)$ and $i \geq 0$. Thus, when Eloise uses $\sigma_{\mathrm{E}}$, she will either stay at states $q^{\prime}$ where $\mathcal{M}, q^{\prime} \mid=\theta$ for infinitely long or reach a state $q^{\prime}$ where $\mathcal{M}, q^{\prime} \mid=\psi$ at some point. Hence, either (1) the embedded game continues infinitely long, whence Eloise wins the whole evaluation game, or (2) by the inductive hypothesis, the exit position is a winning position for Eloise. Therefore, we have that $\mathcal{M}, q \mid={ }_{u}^{g}\langle A \lambda\rangle \psi \mathrm{R} \theta$.

Suppose, then, that $\mathcal{M}, q \mid=_{u}^{g}\left\langle\langle A\rangle \psi \mathrm{R} \theta\right.$, that is, Eloise has a winning strategy $\Sigma_{\mathrm{E}}$ from the position $\left(\mathbf{E}, q,\langle\langle A\rangle \psi \mathrm{R} \theta)\right.$. Now, $\Sigma_{\mathbf{E}}\left(\mathbf{E}, q,\langle\langle A\rangle \psi \mathrm{R} \theta)\right.$ is a strategy $\sigma_{\mathbf{E}}$. Let $S_{A}$ be the collective strategy that is related to $\sigma_{\mathrm{E}}$ and let $\Lambda \in$ paths $\left(q, S_{A}\right)$. Now, when Eloise uses $\sigma_{\mathrm{E}}$, there exist actions of Abelard such that the states of the embedded game are on $\Lambda$ (until a state is reached where Eloise ends the game-if such a state exists). We need to show that for every $i \geq 0$ either $\mathcal{M}, \Lambda[i] \mid=\theta$ or there is $j<i$ such that $\mathcal{M}, \Lambda[j] \mid=\psi$. Let $i \geq 0$. If Eloise ends the game before $i$ rounds, the game ends at an exit position $(\mathbf{E}, \Lambda[j], \psi)$ for some $j<i$, and we can conclude that $\mathcal{M}, \Lambda[j]=\psi$ by the inductive hypothesis. If Eloise does not end the game before $i$ rounds have been played, then Abelard can end it in the exit position $(\mathrm{E}, \Lambda[i], \theta)$. We can then conclude that $\mathcal{M}, \Lambda[i] \mid=\theta$ by the inductive hypothesis. Therefore, $\mathcal{M}, q \mid=\langle\langle A\rangle \psi \mathrm{R} \theta$.

This completes the proof of the theorem.

\section{Proof of Theorem 5.6}

By Lemma 5.5, we may assume for this proof that all of the (winning) strategies that Eloise uses in embedded games depend on states only. This amounts to assuming that their domain is the set of states instead of configurations. We also recall that timers are not needed in the finitely bounded case. We show by induction on $\varphi$ that the following holds for every $q \in$ St.

$$
\mathcal{M},\left.q\right|_{f} \varphi \text { iff } \mathcal{M},\left.q\right|_{f} ^{g} \varphi .
$$

- The cases in which $\operatorname{Pos}=(\mathbf{P}, q, p)(p \in \Pi), \operatorname{Pos}=(\mathbf{P}, q, \neg \psi), \operatorname{Pos}=(\mathbf{P}, q, \psi \vee \theta)$, or Pos $=$ $(\mathbf{P}, q,\langle\langle A\rangle\rangle \mathrm{X} \psi)$ are treated exactly as in the proof of Theorem 5.1.

- Suppose that $\varphi=\langle\langle A\rangle \psi \cup \theta$.

Suppose first that $\mathcal{M},\left.q\right|_{f}\left\langle\langle A\rangle \psi \cup \theta\right.$, that is, there exist some $n<\omega$ and $S_{A}$ such that for each $\Lambda \in$ paths $\left(q, S_{A}\right)$, there is some $i \leq n$ such that $\mathcal{M},\left.\Lambda[i]\right|_{f} \theta$ and $\mathcal{M}, \Lambda[j]||_{f} \psi$ for every $j<i$. Let $\Sigma_{\mathrm{E}}(\mathrm{E}, q,\langle\langle A\rangle\rangle \psi \cup \theta)=\left(n, \sigma_{\mathrm{E}}\right)$, where $\sigma_{\mathrm{E}}\left(q^{\prime}\right)=\theta$ for each $q^{\prime} \in$ St where $\mathcal{M},\left.q^{\prime}\right|_{f} \theta$ and for all other states $q^{\prime} \in$ St, let $\sigma_{\mathrm{E}}\left(q^{\prime}\right)$ be the tuple of actions for the agents in $A$ chosen according to $S_{A}$. Now, when Eloise chooses the time limit to be $n$ and uses $\sigma_{\mathrm{E}}$, then, regardless of the actions of Abelard, all states that are reached in the game must be states $\Lambda[i]$ for some $\Lambda \in \operatorname{paths}\left(q, S_{A}\right)$ and $i \leq n$. Thus, Eloise can reach a state $q^{\prime}$ where $\mathcal{M},\left.q^{\prime}\right|_{f} \theta$ 
in $n$ rounds; if Abelard ends the game before that at some state $q^{\prime}$, then $\mathcal{M},\left.q\right|_{f} \psi$. By the inductive hypothesis, either of these cases will result in an exit position that is a winning position for Eloise. Therefore, we have that $\mathcal{M}, q \models_{f}^{g}\langle\langle A\rangle \psi \cup \theta$.

Suppose, then, that $\mathcal{M},\left.q\right|_{{ }_{f}^{g}} ^{g}\left\langle\langle A\rangle \psi \cup \theta\right.$, that is, Eloise has a winning strategy $\Sigma_{\mathrm{E}}$ from the position $(\mathrm{E}, q,\langle\langle A\rangle\rangle \psi \cup \theta)$. Now, $\Sigma_{\mathrm{E}}(\mathrm{E}, q,\langle\langle A\rangle\rangle \psi \cup \theta)=\left(n, \sigma_{\mathrm{E}}\right)$, where $n<\omega$. Let $S_{A}$ be the collective strategy that is related to the strategy $\sigma_{\mathrm{E}}$ (see the corresponding part in the proof of Theorem 5.1). Let $\Lambda \in$ paths $\left(q, S_{A}\right)$. Now, when Eloise uses $\sigma_{\mathrm{E}}$, there exist actions of Abelard such that the states of the embedded game will be on $\Lambda$ until some configuration $(n-i, \Lambda[i])$ (with $i \leq n)$ at which Eloise ends the game at the position $(\mathrm{E}, \Lambda[i], \theta)$. (If she does not end the game, then it will automatically end at the exit position $(E, \Lambda[n], \theta)$.) Since $\Sigma_{\mathbf{E}}$ is a winning strategy, we have that $\mathcal{M}, \Lambda[i] \mid=_{f} \theta$ by the inductive hypothesis. Then, let $j<i$. Since Abelard can end the game after $j$ rounds at the position $(\mathbf{E}, \Lambda[j], \psi)$, by the inductive hypothesis, we have that $\mathcal{M},\left.\Lambda[j]\right|_{f} \psi$. Hence, $\mathcal{M},\left.q\right|_{f}\langle\langle A\rangle \psi \cup \theta$.

- Suppose that $\varphi=\langle\langle A\rangle\rangle \mathrm{R} \theta$.

Suppose first that $\mathcal{M},\left.q\right|_{f}\langle\langle A\rangle \psi \mathrm{R} \theta$, that is, for all $n<\omega$, there exists a collective strategy $S_{A, n}$ such that for each $\Lambda \in$ paths $\left(q, S_{A, n}\right)$ and $i \leq n$, we have either that $\mathcal{M}, \Lambda[i] \mid=_{f} \theta$ or that there is some $j<i$ such that $\mathcal{M},\left.\Lambda[j]\right|_{f} \psi$. Let $\Sigma_{\mathbf{E}}(\mathbf{E}, q,\langle\langle A\rangle \psi \cup \theta)$ be a function that maps every $n<\omega$ to $\sigma_{\mathrm{E}, n}$, where $\sigma_{\mathrm{E}, n}$ is defined as follows. Let $\sigma_{\mathrm{E}, n}\left(q^{\prime}\right)=\psi$ for each $q^{\prime} \in$ St where $\mathcal{M},\left.q^{\prime}\right|_{f} \psi$. For all other states $q^{\prime} \in$ St, let $\sigma_{\mathrm{E}, n}\left(q^{\prime}\right)$ be the tuple of actions for the agents in $A$ chosen according to $S_{A, n}$. Now, when Eloise uses $\sigma_{\mathrm{E}, n}$, all states that can be reached must be states $\Lambda[i]$ for some $\Lambda \in$ paths $\left(q, S_{A}\right)$ and $i \leq n$. Thus, Eloise will either stay at states $q^{\prime}$ where $\mathcal{M},\left.q^{\prime}\right|_{f} \theta$ for $n$ rounds or reach a state $q^{\prime}$ where $\mathcal{M},\left.q^{\prime}\right|_{f} \psi$ while maintaining the truth of $\theta$. By the inductive hypothesis, either of these cases will result in an exit position that is a winning position for Eloise. Therefore, we have that $\mathcal{M},\left.q\right|_{f} ^{g}\langle\langle A\rangle \psi \mathrm{R} \theta$.

Suppose, then, that $\mathcal{M}, q \models_{f}^{g}\left\langle\langle A\rangle \psi \mathrm{R} \theta\right.$, that is, Eloise has a winning strategy $\Sigma_{\mathrm{E}}$ from the position $(\mathbf{E}, q,\langle\langle A\rangle\rangle \mathrm{R} \theta)$. Now, $\Sigma_{\mathrm{E}}\left(\mathbf{E}, q,\langle\langle A\rangle \psi \mathrm{R} \theta)\right.$ assigns some strategy $\sigma_{\mathrm{E}, n}$ for every $n<\omega$. Let $n<\omega$ and let $S_{A, n}$ be the collective strategy that is related to $\sigma_{\mathrm{E}, n}$. Let $\Lambda \in$ paths $\left(q, S_{A, n}\right)$. Now, when Eloise plays using $\sigma_{\mathrm{E}, n}$, there are some actions of Abelard such that the states of the embedded game are on $\Lambda$ until Eloise ends the game or $n$ rounds have lapsed. We need to show that for every $i \leq n$, either $\mathcal{M}, \Lambda[i] \mid \models_{f} \theta$ or there is some $j<i$ such that $\mathcal{M},\left.\Lambda[j]\right|_{f} \psi$. Let $i \leq n$. If Eloise ends the game before $i$ rounds have gone, the game ends at the position $(\mathrm{E}, \Lambda[j], \psi)$ for some $j<i$. Then, by the inductive hypothesis, $\mathcal{M},\left.\Lambda[j]\right|_{f} \psi$. If Eloise does not end the game before $i$ rounds have lapsed, then Abelard may end it at the position $(\mathrm{E}, \Lambda[i], \theta)$, whence, by the inductive hypothesis, $\mathcal{M},\left.\Lambda[i]\right|_{f} \theta$. Therefore, $\mathcal{M},\left.q\right|_{f}\langle\langle A\rangle \psi \mathrm{R} \theta$.

This completes the proof of the theorem.

\section{ACKNOWLEDGMENTS}

We thank the anonymous reviewers for helpful comments and references.

\section{REFERENCES}

Natasha Alechina, Nils Bulling, Brian Logan, and Hoang Nga Nguyen. 2015. On the boundary of (un)decidability: Decidable model-checking for a fragment of resource agent logic. In IFCAI'15. AAAI Press, Palo Alto, California USA, 1494-1501.

Natasha Alechina, Brian Logan, Nguyen Hoang Nga, and Abdur Rakib. 2011. Logic for coalitions with bounded resources. Journal of Logic and Computation 21, 6, 907-937.

Shaull Almagor, Yoram Hirshfeld, and Orna Kupferman. 2010. Promptness in omega-regular automata. In Proceedings of of ATVA'10, Lecture Notes in Computer Science, Ahmed Bouajjani and Wei-Ngan Chin (Eds.), Vol. 6252. Springer, Berlin, $22-36$. 
Rajeev Alur and Thomas A. Henzinger. 1998. Finitary fairness. ACM Transactions on Programing Languages and Systems 20, 6, 1171-1194.

R. Alur, T. A. Henzinger, and O. Kupferman. 2002. Alternating-time temporal logic. fournal of the ACM 49, 5, 672-713.

Benjamin Aminof, Vadim Malvone, Aniello Murano, and Sasha Rubin. 2016. Graded strategy logic: Reasoning about uniqueness of Nash equilibria. In Proceedings of AAMAS'16. IFAAMAS Publications, 698-706.

Petr Cermák, Alessio Lomuscio, and Aniello Murano. 2015. Verifying and synthesising multi-agent systems against onegoal strategy logic specifications. In Proceedings of AAAI'15, Blai Bonet and Sven Koenig (Eds.). AAAI Press, 2038-2044.

Krishnendu Chatterjee, Thomas A. Henzinger, and Florian Horn. 2009. Finitary winning in omega-regular games. ACM Transactions on Computational Logic 11, 1, 1:1-1:27.

Corina Cîrstea, Clemens Kupke, and Dirk Pattinson. 2009. EXPTIME Tableaux for the coalgebraic $\mu$-calculus. In Proceedings of CSL'09, Lecture Notes in Computer Science, Vol. 5771. Springer, Berlin, 179-193.

Corina Cîrstea, Alexander Kurz, Dirk Pattinson, Lutz Schröder, and Yde Venema. 2011. Modal logics are coalgebraic. Computer fournal 54, 1, 31-41.

E. Allen Emerson and Charanjit S. Jutla. 1988. The complexity of tree automata and logics of programs (extended abstract). In 29th Annual Symposium on Foundations of Computer Science, White Plains, New York, USA, October 24-26, 1988. 328-337.

E. Allen Emerson, Aloysius K. Mok, A. Prasad Sistla, and Jai Srinivasan. 1992. Quantitative temporal reasoning. Real-Time Systems 4, 4, 331-352.

Gaëlle Fontaine, Raul Andres Leal, and Yde Venema. 2010. Automata for coalgebras: An approach using predicate liftings. In Proceedings of ICALP'10, Lecture Notes in Computer Science, Vol. 6199. Springer, Berlin, 381-392.

Valentin Goranko, Antti Kuusisto, and Raine Rönnholm. 2016. Game-theoretic semantics for alternating-time temporal logic. In Proceedings of AAMAS'16. IFAAMAS Publications, 671-679.

Valentin Goranko, Antti Kuusisto, and Raine Rönnholm. 2017a. CTL with finitely bounded semantics. In Proceedings of TIME'17 (LIPIcs), Vol. 90. Schloss Dagstuhl - Leibniz-Zentrum fuer Informatik, 14:1-14:19.

Valentin Goranko, Antti Kuusisto, and Raine Rönnholm. 2017b. Game-theoretic semantics for ATL+ with applications to model checking. In Proceedings of AAMAS'17. IFAAMAS Publications, 1277-1285.

Valentin Goranko, Antti Kuusisto, and Raine Rönnholm. 2018. Alternating-time temporal logic ATL with finitely bounded semantics. submitted.

V. Goranko and G. van Drimmelen. 2006. Complete axiomatization and decidability of alternating-time temporal logic. Theoretical Computer Science 353, 93-117.

Lauri Hella, Antti Kuusisto, and Raine Rönnholm. 2017. Bounded game-theoretic semantics for modal mu-calculus. CoRR abs/1706.00753.

Jaakko Hintikka. 1973. Logic, Language-games and Information: Kantian Themes in the Philosophy of Logic. Clarendon Press, Oxford.

Jaakko Hintikka and Gabriel Sandu. 1989. Informational independence as a semantical phenomenon. In Logic, Methodology and Philosophy of Science VIII, J. E. Fenstad (Ed.). North-Holland, Amsterdam, 571-589.

Jaakko Hintikka and Gabriel Sandu. 1997. Game-theoretical semantics. In Handbook of Logic and Language, J. van Benthem and A. ter Meulen (Eds.). Elsevier, Amsterdam, 361-410.

Orna Kupferman, Nir Piterman, and Moshe Y. Vardi. 2007. From liveness to promptness. Lecture Notes in Computer Science, Werner Damm and Holger Hermanns (Eds.). Vol. 4590. Springer, Berlin, 406-419.

Paul Lorenzen. 1961. Ein dialogisches Konstruktivitätskriterium. In Proceedings of the Symposium on Foundations of Mathematics, Warsaw 1959, Andrzej Mostowski (Ed.). Panstwowe wydawnictwo naukowe, Warsaw, 193-200.

Fabio Mogavero, Aniello Murano, and Luigi Sauro. 2014. Strategy games: A renewed framework. In Proceedings of AAMAS'14, Ana L. C. Bazzan, Michael N. Huhns, Alessio Lomuscio, and Paul Scerri (Eds.). IFAAMAS/ACM, 869-876.

Fabio Mogavero, Aniello Murano, and Loredana Sorrentino. 2013. On promptness in parity games. In Proceedings of LPAR19, Lecture Notes in Computer Science, Kenneth L. McMillan, Aart Middeldorp, and Andrei Voronkov (Eds.), Vol. 8312. Springer, Berlin, 601-618.

Dario Della Monica, Margherita Napoli, and Mimmo Parente. 2011. On a logic for coalitional games with priced-resource agents. Electronic Notes in Theoretical Computer Science 278, 215-228.

Cedrick Smith. 1966. Graphs and composite games. Journal of Combinatorial Theory 1, 51-81.

Yde Venema. 2006. Automata and fixed point logic: A coalgebraic perspective. Information and Computation 204, 4, 637-678.

Received March 2017; revised October 2017; accepted January 2018 